University of Redlands

\title{
Route Optimization and Analysis Register - A Prototype Tool to Solve Travelling Salesman Problem and Compare Recommended and Actual Routes
}

\author{
A Major Individual Project submitted in partial satisfaction of the requirements \\ for the degree of Master of Science in Geographic Information Systems \\ by \\ Arunabha Chowdhury \\ Ruijin MA, Ph.D., Committee Chair \\ Mark Kumler, Ph.D.
}

August 2015 

Route Optimization and Analysis Register - A Prototype Tool to Solve Travelling Salesman Problem and Compare Recommended and Actual Routes

Copyright (C) 2015

by

Arunabha Chowdhury 

The report of Arunabha Chowdhury is approved.

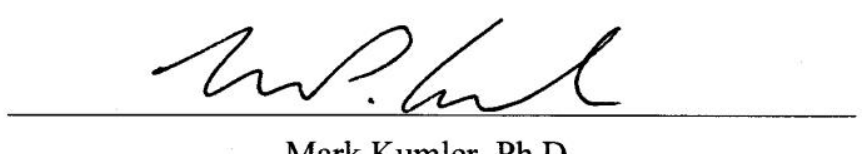

Mark Kumler, Ph.D.

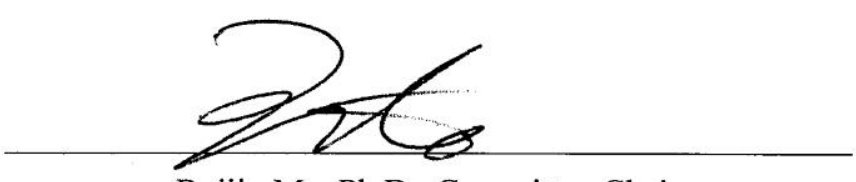

Ruijin Ma, Ph.D., Committee Chair

August 2015 



\section{Acknowledgements}

I would like to express my gratitude to Johnson Kosgei from Red Bull for suggesting this project to the University of Redlands MS GIS program. Thank you to all the Esri instructors who shared their knowledge, passion, and real world GIS experiences. Special acknowledgements to my advisor Dr. Ruijin Ma for guidance throughout the project and pushing me beyond my threshold to complete the project on time. Without Dr. Mark

Kumler, it would have been impossible to find the smallest errors in the report and poster. I have immense respect for Dr. Douglas M. Flewelling, a mentor who saw enough potential in me and suggested me to be a part of the MS GIS program. I would also like to thank Dr. Fang Ren for teaching and passing the knowledge of my favorite class, Statistics. I am grateful to Nathan Strout for teaching me the cool things that can be done using Python.

I believe without Ruben Ortiz providing valuable help whenever my computer decided to act up, it would have been impossible to deliver this project. I always knew I was not a great writer, but did not know writing would be the biggest challenge for this project. I am thankful to Lynn Flewelling for spending enormous time on editing the paper. The only thing that I had to worry about for the last year was studying and staying alive. The rest of the things were taken care of by Andrea Barrios. Thank you so much. It could have been a miserable year without you as the Program Coordinator,

Cohort 26, thanks for all the good times, great memories and long study sessions together. I would like to thank Cohort 23 for inspiring, Cohort 24 for sharing the fun year during my MBA program, Cohort 25 for guiding me towards the MS GIS program, and Cohort 27 for motivating. Special thanks to Nicholas Dunn, Giovan Alcala, Meghan Spicer Alcala, Karina Zavaleta-Abel, Jian Sim, René Pretorius, Matthew Flewelling, John Miller, and everyone's little angel Qian Hui.

I would like to thank my mom, Nandini Chowdhury, and dad, Smarajit Chowdhury, for all their support and encouragement throughout my life. Mom, you are the finest women I have ever seen who always prayed for my safety, happiness, and success. Dad, you are the institution who taught me not regret any decision and always look forward. Everything I do well in my life will be the result of your exceptional parenting. Amar pronam niyo aar ashirbaad koro jaate tomader ke gorbito korte paari (I pay my regards to you both and keep blessing me so that I can always make you both proud).

Last but not the least, a big hug and thank you to my girlfriend, Shilpi Jain for believing in me during all the highs and lows over the past year. I know we will complement each other during every step we take hereafter. Never, ever change. 



\begin{abstract}
Route Optimization and Analysis Register - A Prototype Tool to Solve Travelling Salesman Problem and Compare Recommended and Actual Routes

by

Arunabha Chowdhury

Distribution routing plays a major role in business profit maximization. Continuous route optimization is very challenging for an organization because of frequent changes in customer configuration. Simultaneously, it is important to know how the actual routes performed when compared to the recommended routes. Route Optimization and Analysis Register (ROAR) is an automated solution that daily generates recommended and actual routes, compares the routes to calculate key performance indicators, and shares the results on a dashboard. The process saves time and reduces cost. Organizations that routinely distribute products to consumers can use ROAR to increase their efficiency.
\end{abstract}





\section{Table of Contents}

Chapter 1 - Introduction ............................................................................................................. 1

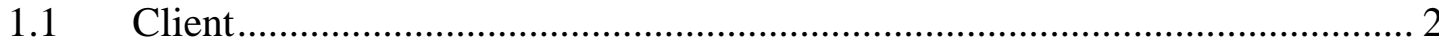

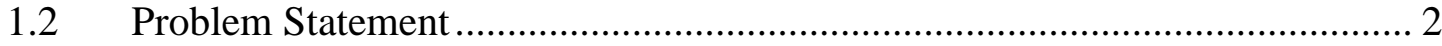

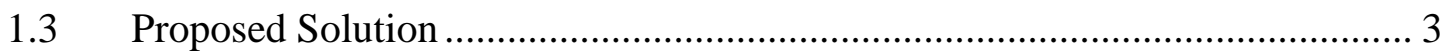

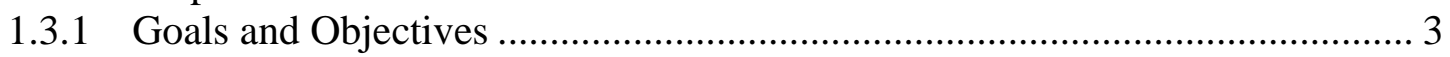

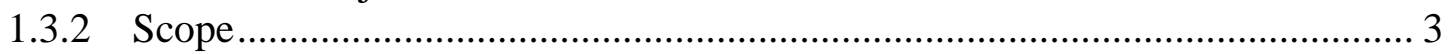

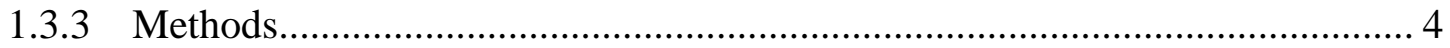

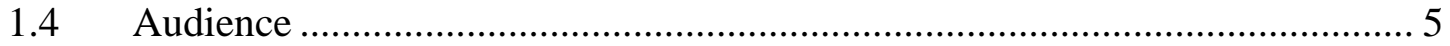

1.5 Overview of the Rest of this Report ........................................................... 5

Chapter 2 - Background and Literature Review ..................................................... 7

$2.1 \quad$ Product Distribution...............................................................................

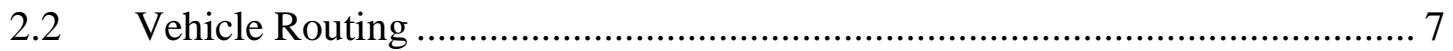

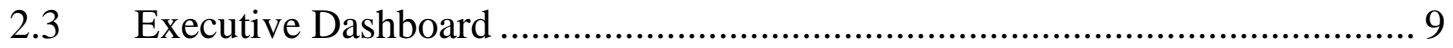

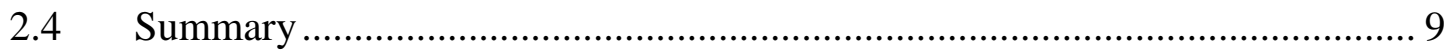

Chapter 3 - Systems Analysis and Design......................................................................... 11

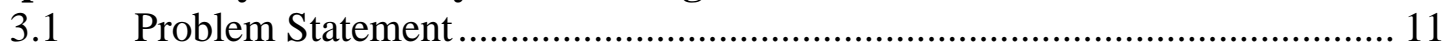

3.2 Requirements Analysis ............................................................................. 11

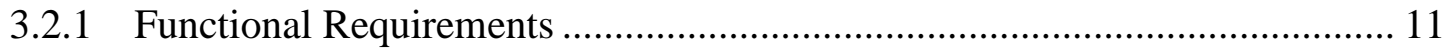

3.2.2 Non-Functional Requirements ................................................................. 12

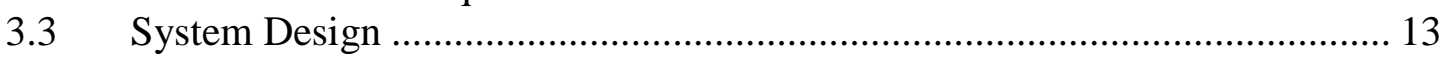

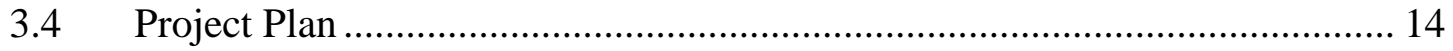

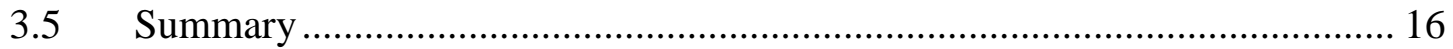

Chapter 4 - Database Design................................................................................................. 17

4.1 Conceptual Data Model ...................................................................... 17

4.2 Logical Data Model ……………....................................................... 18

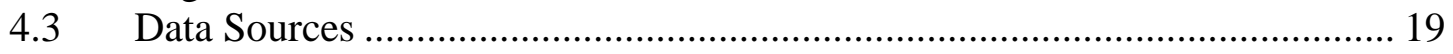

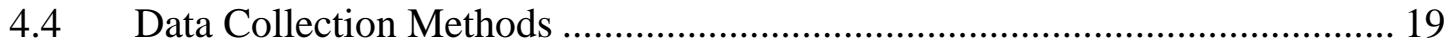

4.5 Data Scrubbing and Loading ……………….......................................... 19

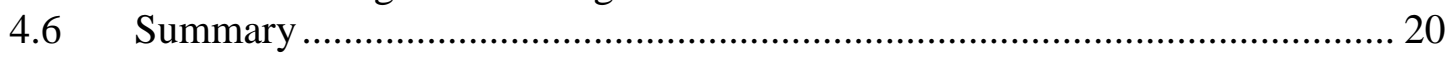

Chapter 5 - Implementation..................................................................................................... 21

5.1 Creating Feature Classes in the Geodatabase ............................................... 21

$5.2 \quad$ Recommended Route ..................................................................... 21

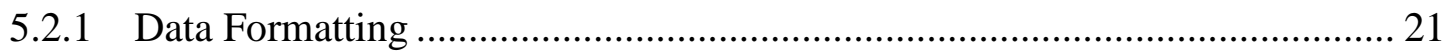

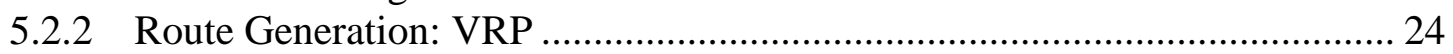

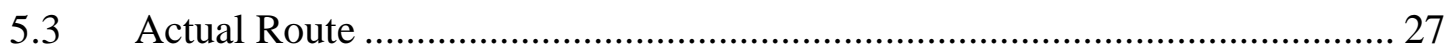

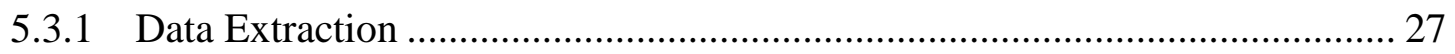

5.3.2 Route Identification ........................................................................ 28

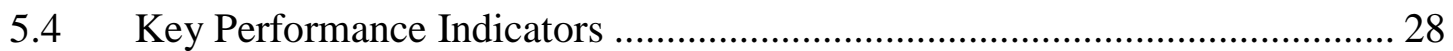

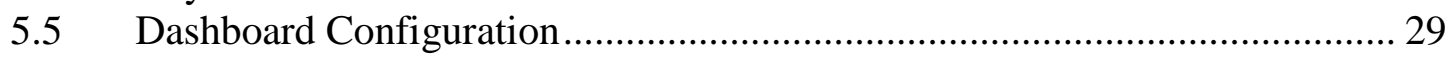

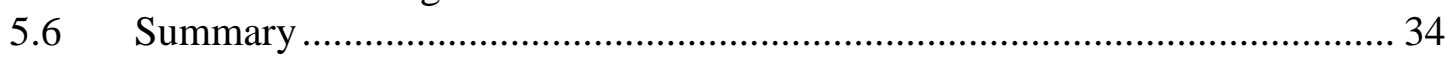

Chapter 6 - Use Case and Discussion ............................................................................. 35

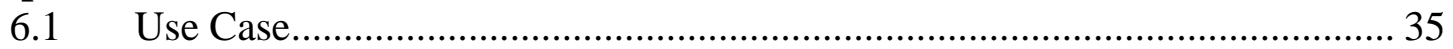

6.1.1 Recommended Route ............................................................................. 35 


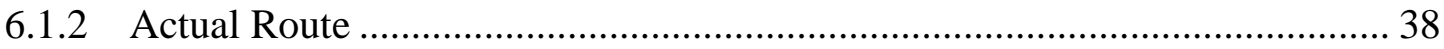

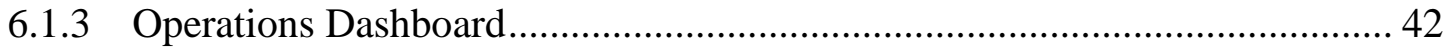

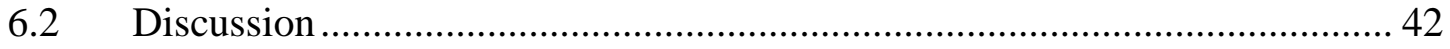

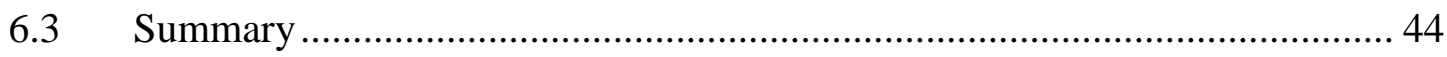

Chapter 7 - Conclusions and Future Work ...................................................................... 45

Works Cited................................................................................................................... 47

Appendix A. Input Data for Recommended Route ....................................................... 49

Appendix B. Recommended Route Generated on May 14, 2015 ............................ 51

Appendix C. Actual Route Generated on May 14, 2015 .......................................... 53

Appendix D. Key Performance Indicators Table on May 14, 2015 ...................... 55

Appendix E. Script for Creating Feature Classes in the Geodatabase ................... 57

Appendix F. Script for Creating Recommended Routes .......................................... 61

Appendix G. Script for Creating Actual Routes ..................................................... 69 


\section{Table of Figures}

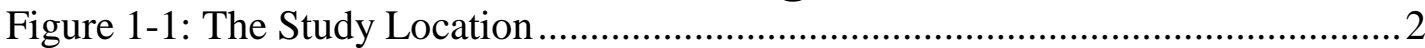

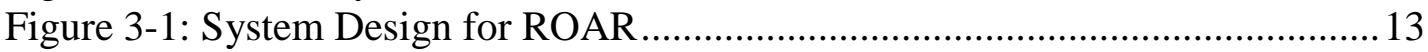

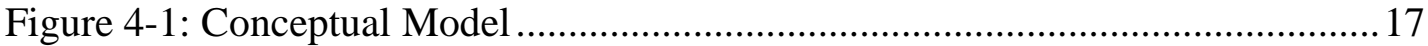

Figure 4-2: Logical Data Model for the File Geodatabase ......................................... 18

Figure 5-1: Vehicle Routing Problem Solver Detailed Workflow ..............................25

Figure 5-2: Warehouse and Customer Locations in Orange County ...........................26

Figure 5-3: Flowchart for Data Extraction from Zonar Web Services......................2 27

Figure 5-4: Scheduling of Recommended Routes Generation. ..................................2 29

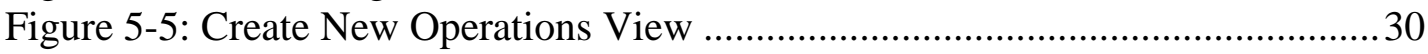

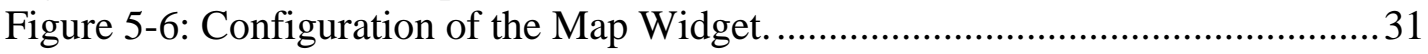

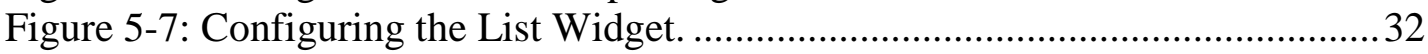

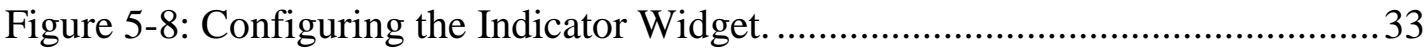

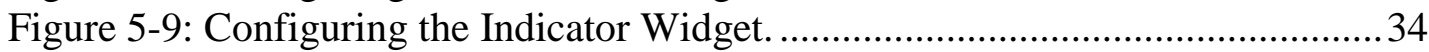

Figure 5-10: Configuring the Dashboard .................................................................. 34

Figure 6-1: Display Message While Generating Recommended Routes......................35

Figure 6-2: Total Customers and Those to be Serviced on Thursday in Week 3 .......36

Figure 6-3: Recommended Routes for the Use Case …………..................................37

Figure 6-4: Recommended Route and Customers for Route ID 001 ..........................38

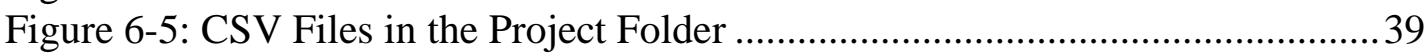

Figure 6-6: Actual Routes for the Use Case ............................................................. 41

Figure 6-7: Actual Route and Corresponding Customers for Route ID 011 .............41

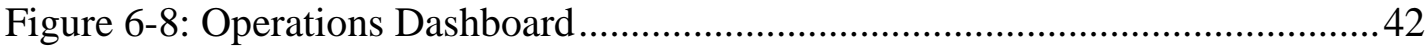

Figure 6-9: GPS Points Identified Incorrectly .......................................................... 43

Figure 6-10: Route Created by Connecting the GPS Points ........................................ 44 



\section{List of Tables}

Table 1. System Design Functional Requirements .................................................... 12

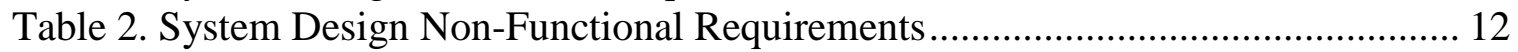

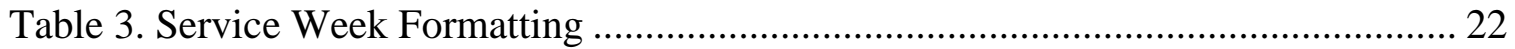

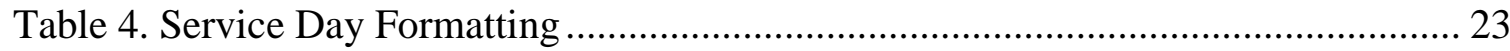

Table 5. Volume Based on the Service Week Pattern .................................................... 24

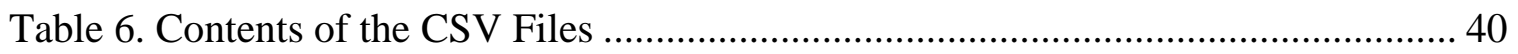

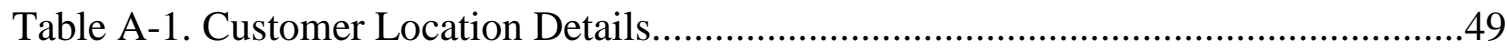

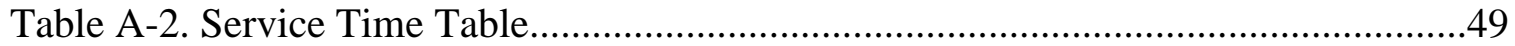

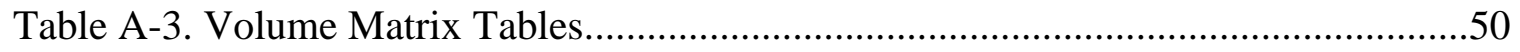

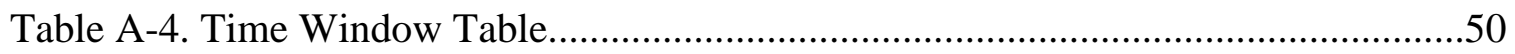

Table B-1. Recommended Stops Feature Class.....................................................51

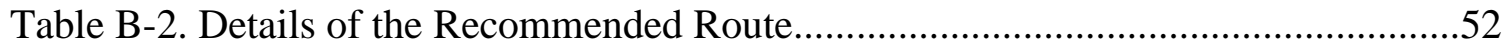

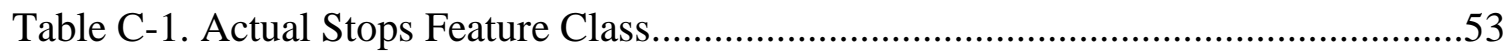

Table C-2. Details of the Actual Route.........................................................................54

Table D-1. An Example of Key Performance Indicators..............................................55 



\section{List of Acronyms and Definitions}

CSV Comma Separated Values

CVRP Capacitated Vehicle Routing Problem

FMCG Fast Moving Consumer Goods

$\mathrm{GHz} \quad$ GigaHertz

GIS Geographic Information System

GPS Global Positioning System

KPI Key Performance Indicators

RAM Random Access Memory

ROAR Route Optimization and Analysis Register

TSP Travelling Salesman Problem

URL Uniform Resource Locator

VRP Vehicle Routing Problem

VRPPD VRP with Pick up and Delivery

VRPTW VRP with Time Windows

XML Extensible Markup Language 



\section{Chapter 1 - Introduction}

Fast Moving Consumer Goods (FMCG) companies operate at a quick pace and sell products with a short shelf life at a relatively low cost. Examples include products such as soft drinks, food, and toiletries, and the shelf life can range anywhere from a few days to a year (Elzakker, Zondervan, Raikar, Hoogland \& Grossmann, 2014). FMCGs have a short shelf life due to either high consumer demand or quick deterioration. This results in a high turnover rate of product. In order to maintain both a high profit margin and good customer relationships, these companies must keep a healthy inventory of product both on the shelf and as back stock. Keeping goods stocked in several different stores has proven to be the biggest challenge for any FMCG company. To ensure on-time delivery of goods, distributors must provide drivers with optimized delivery routes.

Although marketing teams and sales teams differ in terms of activities, they share the same goal: to increase business profits. The marketing and sales teams of the Red Bull Corporation strive to maximize their profits. Both teams must consider basic routing factors: where are the distribution centers, how easy are they to access, and how much does it cost? The answers to these questions allowed the development of optimal routes for drivers.

The recommended route of a distribution center plays a major role in the profit maximization of a business. Red Bull Corporation serves a large number of stores on a regular basis. In order for its product to be delivered quickly and efficiently, the company needed to know the best route to the customers. California has several Red Bull warehouses, including one in Orange County, and serves the stores within the county and the surrounding cities of Placentia, Signal Hill, Lakewood, Artesia, Long Beach, Cerritos, and Hawaiian Gardens in Los Angeles County (Figure 1-1). According to a senior sales official, at present the study area serves approximately 2,000 customers and is projected to reach up to 7,000 customers. Red Bull intends to maintain its leadership position, as reflected in their mission statement "To spread our wings over the world" as it continues to focus on global expansion (Costley, Ruiz, Shi, Wong \& Huynh, 2013). 


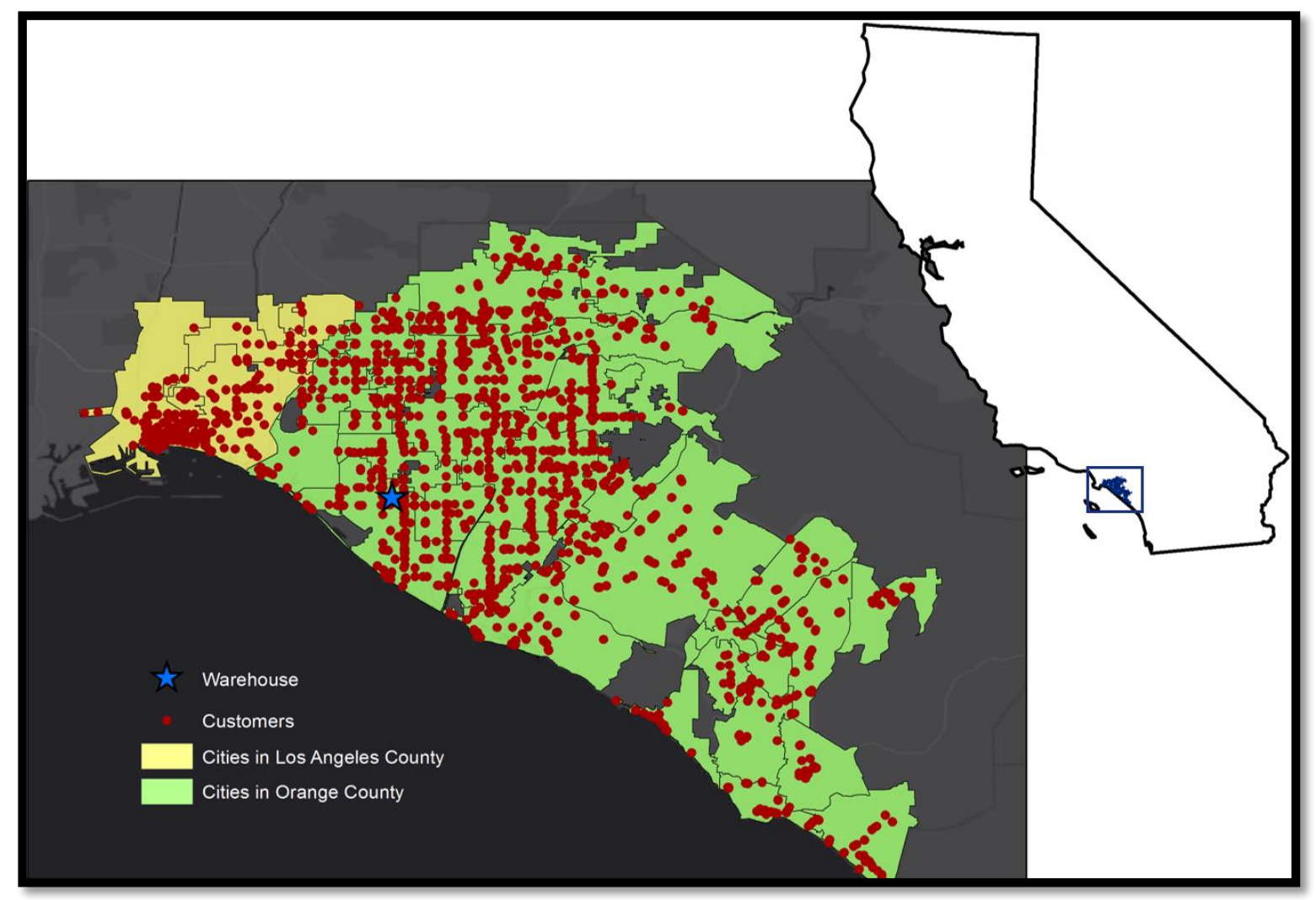

Figure 1-1: The Study Location

This chapter provides the background for this project. Section 1.1 introduces the client. Section 1.2 addresses the problem that the organization was experiencing. Section 1.3 provides a summary of the proposed solution. Section 1.4 describes the intended audience. Finally, Section 1.5 outlines the rest of this document.

\subsection{Client}

The client for this project was Red Bull Corporation. The organization was founded on the April 1, 1987 drawing inspiration from the energy drinks of the Far East. Their products are now sold in more than 160 countries worldwide (Red Bull Corporation, 2014). The point of contact was Mr. Johnson Kosgei, GIS Specialist for the corporation. Mr. Kosgei currently leads the design and implementation of GIS initiatives and innovations that support Red Bull's decision-making and business operations.

\subsection{Problem Statement}

Route optimization at Red Bull has been conducted only twice a year, primarily because it is an expensive manual process. Because of the long interval between the route optimization process and the frequent changing customer configurations, routes often became inefficient after approximately one month. On average, there are 500 customer configuration changes per month due to either addition or elimination. These changes 
impact the recommended optimal efficiency. In addition, the organization did not have a tool to analyze the performance of a route that a truck actually followed, in comparison to the optimized recommended route.

\subsection{Proposed Solution}

Route Optimization and Analysis Register, hereafter known as ROAR, was to be an automated system that would reduce the manual labor aspect of conducting the route optimization process. Factors that contributed to the overall cost and profitability were taken into account in order to generate optimized routes. As a suggested solution to the problem, the ROAR system would run more frequently than the previous system, generating reports that are more accurate, and suggesting updated routings for better service delivery and profit.

The proposed solution for this project was to create a route generation toolset to help Red Bull customize the relevant factors and calculate optimized routes on a daily basis. Once the optimal recommended routes were created, the tool had to extract the GPS points from an Extensible Markup Language (XML) web service hosted by a third party fleet management system called Zonar, and then generate an approximation of the actual routes. The analysis between recommended routes and actual routes would be displayed on a dashboard.

\subsubsection{Goals and Objectives}

The goal of this project was to create a routing tool that could run on a daily basis to generate optimized routes for the Red Bull distribution trucks and provide analysis of recommended routes and the approximate actual routes. The first step of the project was to determine the objectives, which helped to create the project plan. There were three objectives of the project:

The first objective was to create the recommended routes and approximate actual routes using ArcGIS. The recommended routes were determined before the trucks left the warehouse. The approximate of actual routes were generated after the trucks returned to the warehouse. The second objective was to increase the efficiency of the recommended route generation by automating the processes so that ROAR could run on a daily basis. The final objective was to provide an interface that could display the actual and the recommended routes on a dashboard with the analysis results.

\subsubsection{Scope}

The study area included Orange County and the cities of Placentia, Signal Hill, Lakewood, Artesia, Long Beach, Cerritos, and Hawaiian Gardens in Los Angeles County. The scope of the project was to use a detailed road network dataset for the study area that was obtained from an Esri business analyst license. The results from the recommended routes were examined and compared to the approximate actual routes. The client provided customer location details for recommended routes, while the data for the actual routes were available on Zonar's dashboard for Red Bull. Results were displayed 
on the new dashboard that was created as a part of this project. Dividing the study area into several service areas was out of scope. The Red Bull GIS team provided the predefined service areas.

Certain requirements or drawbacks discussed by the client narrowed the scope and added limitations. For sharing the results, a configurable dashboard was required. In order to do so, the client insisted on using Esri's Operations Dashboard. Addition or deletion from the customer list was out of the scope for this project; the Red Bull GIS team is responsible for adding or removing customers from the customer list manually. Any Red Bull truck can serve up to 25 customers. There might be instances where a zone has more than 25 customers, and in such cases only 25 customers would be served. After discussion with the client, it was decided that solving this issue was out of scope. The access to actual customers served was not provided due to confidentiality reasons. As a result, it was decided that any stop of more than 90 seconds would be considered as a customer being served. Although this assumption did not confirm the results as completely correct, it was decided that the exact result generation for the customer stops would be out of scope.

\subsubsection{Methods}

The software requirement for this project included Esri's ArcGIS Desktop 10.2.2, with the Network Analyst and Business Analyst extensions enabled. The Network Analyst Vehicle Routing Problem solver was used to perform route calculations. Other important software suites used were ArcGIS Online, ArcGIS Server, Esri's Operations Dashboard, PyScripter, and Microsoft Office. An Agile project management approach was used for this study. The major steps for this project were the planning phase, the implementation phase, and the finalization phase.

The planning phase of the project involved discussions with the client to identify the initial sets of requirement analysis. During this phase, factors were identified that helped create the routing model. Different ways to collect the datasets were verified during the discussions. The required datasets were extracted from the client's system or different third party systems.

The implementation phase involved regular phone and WebEx meetings with the client to discuss discrepancies in the datasets. Through intensive research, the corrupted parts of datasets were cleaned in order to use the routing tool. Additions to the datasets after data formatting were approved and completed. This phase required configuring the data, identifying different network parameters, executing the solver analysis, and repeating the steps as needed until the desired results were achieved.

The finalization phase included the implementation of the product. During this phase the ROAR results were reviewed, procedures were documented, and the product was transferred to the client with a detailed document containing working instructions.

Creating a toolset to solve the vehicle routing problem (VRP) was one of the major deliverables of the ROAR project. Optimized routes considered factors like service 
day, service time, time windows, volume, and truck capacities. To achieve this purpose, the following components were included:

1. Create feature classes to hold the recommended route data provided by client using Microsoft Excel format.

2. Create an automation script to extract actual route data from the Zonar system, a third party fleet management system.

3. Import data into a file geodatabase.

4. Create script that formats the raw data into ArcGIS data format.

5. Create Python scripts that generate the recommended route and approximate actual route.

6. Share output routes in a web map and display the maps and the analysis results on dashboard.

7. Provide the client with a detailed instruction manual that explains the installation and system workflow.

\subsection{Audience}

This report discusses the methodology used using various geographical information system (GIS) software and is intended for GIS professionals with knowledge of ArcGIS and Python. The audience should have an understanding of the Vehicle Routing Problem and Python programming in order to replicate the project. This report is intended to give the reader a better understanding of the terms, procedures, and techniques used throughout the route planning analysis. Different organizations involved in vehicle fleet management that routinely distribute products to consumers can use it to increase their efficiency. They may find interest in the overall concept and benefits of the project.

\subsection{Overview of the Rest of this Report}

The balance of this report discusses the project components, how they were applied, and steps conducted to perform the testing. Chapter 2 discusses the background and literature related to the project. It includes the different routing algorithms that were used in the past to solve problems that dealt with routing for distribution of goods. Chapter 3 explains the systems analysis and design phases of the project, detailing the client's problem. Chapter 4 describes the database design and structure. Chapter 5 demonstrates the implementation process and provides comprehensive understanding of how to build a similar tool. Chapter 6 examines the results and analysis of the project. Chapter 7 contains the findings and future work. 


\section{Chapter 2 - Background and Literature Review}

The use of GIS technology helps organizations implement cost conscious route optimization decisions to keep expenses down. The use of GIS helps identifying cost savings, locating features spatially, and travelling to the destinations more efficiently. A detailed literature review was significant to understand the previous work on similar problems. Routing is an important problem in the fields of transportation, distribution, and logistics (Dantzig \& Ramser, 1959). A routing problem with constraints like delivery volume, time windows, and service time is known as a vehicle routing problem (VRP). The traveling salesman problem (TSP) is the problem of finding a shortest closed tour that visits all the cities in a given set; it is a special case of the VRP (Dorigo \& Gambardella, 1997).

Section 2.1 provides insight into distribution of products in an organization. Section 2.2 outlines the work done in the field of route optimization. Section 2.3 investigates different functionalities of Esri's Operations Dashboard. Finally, Section 2.4 summarizes the findings.

\subsection{Product Distribution}

The ability to obtain an efficient distribution channel is critical to a Fast Moving Consumer Goods (FMCG) company. If a product in demand is not available at the right time, sales will decline, reducing profits for the businesses. Knowing the factors that determine customers' buying decisions should help manufacturers target their marketing activities for the widest profitable distribution. The locations of the customers like restaurants, grocery stores, and retail stores influence the customer buying decision (Montgomery, 1975). These locations of customers are important in order to know if they can be best served together using a single vehicle, or served in smaller groups using multiple vehicles.

Burns, Hall, Blumenfeld, and Daganzo agreed that any analysis should consider all the transportation and inventory costs involved in distributing the items, regardless of the cost allocation methodology (1985). The authors discussed the different factors that an FMCG company should consider while distributing its products. Some of the constraints they listed include delivery volume, service time, time windows, vehicle capacity, travel time, distance between warehouse and a customer, and distance between two customers.

\subsection{Vehicle Routing and Travelling Salesman Problem}

Routing algorithms commonly use the distance calculation with other constraints to determine possible routes to predefined destinations. Different types of algorithms can be used to calculate these paths. Some of the major routing algorithms used in the past are Simulated Annealing, Tabu Search, Genetic Algorithm, Ant Colony Optimization, and Dijkstra's algorithm (Karadimas, Kolokathi, Defteraiou, \& Loumas, 2007). This section outlines the details of Dijkstra's algorithm and VRP. 
Solving VRP allows determining the optimal routes and is helpful to companies that are involved in distributing products or services as their business. According to Kumar and Panneerselvam (2012), solving VRP involves designing an optimal route for a fleet of vehicles to service a set of customers, given a set of constraints. The authors reviewed different VRP applications involving capacitated vehicle routing problem (CVRP), VRP with pick-up and delivery (VRPPD), and VRP with time windows (VRPTW).

A solution to VRP allows fleets of vehicles to serve a set of customers with known demands on minimum-cost vehicle routes originating and terminating at a depot, which meant less time, fewer expenses, and more profit (Chang \& Chen, 2007). The objective function of a VRP can vary depending on the particular application. These include minimizing the total transportation cost based on the total distance travelled, the fixed costs associated with the drivers, and the number of vehicles needed to serve all customers (Carić \& Gold, 2008).

The TSP's most common practical implementation is seeking the shortest path in a defined area for a fixed number of clients. As mentioned in Section 1.3.2, the service areas were pre-defined, thus making the goal of this project a set of solutions multiple TSPs. There are approximately 20 different service areas, thus a complete solution would compute approximately 20 different TSP solutions. Each TSP solution will be a closed loop for a delivery truck from and to the warehouse serving a fixed number of locations with constraints like time windows, service times, and volumes.

The ArcGIS Network Analyst extension has a set of tools that help analysts solve problems including routing, travel directions, closest facility, and service area analysis. It allows users to analyze the network problems in a more realistic way by considering turns, speed limits, street and height restrictions, and traffic conditions at different times of the day (Karadimas et al., 2007). Network Analyst allows a user to generate simple optimized routes, find closest facilities, solve complex multi-vehicle routing problems, perform location allocation, determine service areas, and create origin distance matrices (Esri, n.d.).

Several projects were developed in the past using the Network Analyst VRP solver to calculate the minimum cost path for a fleet of delivery vehicles. O'Conner developed a tool to determine optimal collection routes by matching real world travel conditions and patterns (2013). His work has used VRP solver and Route Solver to determine the differences in the cost of the sanitation collection and transportation. With VRP solver, O'Conner found the results were not reasonable for more than 100 locations, but for 87 locations, better results were identified. This project tried to solve a travelling salesman problem in each predefined service area. It was already known that there were no service areas with more than 30 locations. The extension was used to determine optimal routes for small collection groups providing evidence of the importance of the size of the group. The author outlined the workflow and best practices for future analysis throughout the city. The study proved that the Network Analyst extension could handle multiple variables like service time, time windows, and volume to generate optimized routes. 
Davis generated a model builder toolset with ArcGIS Network Analyst to provide fruit and vegetable vendors with optimal delivery routes (2013). The author provided a method for inputting parameters and setting analysis layer properties to allow a user to generate optimized routes. The tool also considered time windows for different customers, forcing the solver to calculate the route that best served the customers within the period. The tool maintained a track of the total delivery volume in the route. Specialty criteria could be configured for each customer or delivery truck, enabling the solver to consider exceptions for certain customers.

VRP solver allows a user to add delivery time constraints for a customer to determine the service time, travel time, and total time in a route. The VRP solver can assign capacity values to each truck, helping the user learn whether a truck has the capacity to serve a particular route. It also allows users to apply specific network dataset rules to solve the transportation problem. Many rules can be identified with day-to-day product distribution for FMCG companies, and VRP solver can be configured accordingly. Moreover, VRP solver does not require the customers to be served in a specific sequence unless specified, which allows the truck to find the optimal delivery sequence.

\subsection{Executive Dashboard}

A dashboard is an easy-to-understand graphical interface allowing users to monitor different events periodically. Although ArcGIS Online is a key part for GIS organizations and users, a dashboard can display the results to non-GIS users in a simpler format. Users can subscribe to online services such as geocoding, spatial analytics, and infograms, and publish results through dashboards and various smartphone platforms to reach the public, planners, and decision makers (Munnik, 2013). This allows a marketing and distribution team to understand the performance of a delivery truck on an actual route, compared to that on a recommended route. Operations Dashboard for ArcGIS allows a user to monitor services, check work orders, track people, and report events across an organization (Esri, 2015).

The operations dashboard application allows users to study responsive maps and dynamic data, which can then be updated automatically. In a situation like Red Bull's fleet management, where it is important to monitor the performance of the delivery trucks on a daily basis, Operations Dashboard can be a very powerful tool for sharing results. It allows a developer to create and share operational views that include interactive maps, list charts, gauges, and other performance indicators. ArcGIS Online subscribers can install the Operations Dashboard via a click-once deployment without the need for administrator rights (Munnik, 2013). One can easily configure widgets and monitor the fleet performance. It allows a user to filter a long list of events to select the required event, highlight it, and zoom to it on the map.

\subsection{Summary}

VRP solver in ArcGIS Network Analyst extension is an excellent choice to optimize the recommended routes. All the required constraints can be added to the VRP layer in order 
to simulate real time scenarios. Even though VRP is a very complicated problem and computationally it becomes highly intensive, it can still produce successful results overpowering the complexity. Operations Dashboard serves the purpose of the graphical interface, allowing the sales and marketing teams to analyze the performance of the actual routes in comparison to the optimized planed routes. 


\section{Chapter 3 - Systems Analysis and Design}

The success of a project depends on both design and planning. This chapter identifies the project design for the Route Optimization and Analysis Register (ROAR) and discusses the entire project plan and analysis used. The requirements analysis section identifies both functional and non-functional requirements gathered to perform the project analysis. The system design section discusses the software, hardware, and major design components of the project. Finally, the complete description of the project plan explains the project's main tasks.

\subsection{Problem Statement}

Route optimization was conducted only twice per year at Red Bull, primarily because it was both expensive and labor intensive. Because of the long interval between the route optimization process and changing store configurations, routes often became inefficient after only a few weeks. On average, there are 500 customer configuration changes per month within the study area, due to the addition or elimination of a store, change of time window, increase or decrease of demand, and location change of the store. This means the recommended routes sub-optimal after only a few weeks.

\subsection{Requirements Analysis}

Requirement gathering was an important step for defining a solution for the client's problem and completing the goals and deliverables for the project. Requirements are either functional or non-functional. The functional requirements were based on elements of input and output details. The non-functional requirements allowed the developer to look into the technical, operational, and transitional requirements that support the main components, the functional requirements.

\subsubsection{Functional Requirements}

The primary functional requirement was the calculation of the optimized recommended routes for the distribution trucks. Constraints included route zone, time window, service time, service day, volume, and vehicle capacity. Another important requirement was to determine the actual routes based on GPS data from Zonar system, a third party fleet management portal for Red Bull. An automation script was required to extract data from Zonar for the actual routes. The functional requirements are listed in Table 1. In order to create routes, a complete network dataset was needed. Esri Street Dataset from the Business Analyst extension was used. For the analysis and comparison of the actual and the recommended routes, a dashboard was required. 
Table 1. System Design Functional Requirements

\begin{tabular}{|l|l|}
\hline Requirement & Description \\
\hline $\begin{array}{l}\text { Automating Zonar Data } \\
\text { Extraction }\end{array}$ & $\begin{array}{l}\text { An automation script was required, which can extract } \\
\text { the Zonar data on a daily basis. }\end{array}$ \\
\hline $\begin{array}{l}\text { Generating Optimized } \\
\text { recommended Route }\end{array}$ & $\begin{array}{l}\text { Calculation of newly optimized recommended route for } \\
\text { distribution trucks. }\end{array}$ \\
\hline Mapping Actual Route & $\begin{array}{l}\text { Calculation of the approximate route for distribution } \\
\text { trucks based on the point data from Zonar. }\end{array}$ \\
\hline $\begin{array}{l}\text { Identifying the differences } \\
\text { between the routes }\end{array}$ & $\begin{array}{l}\text { Analysis should consider percentage of stores served } \\
\text { (actual vs recommended) and time deviation. It also } \\
\text { needs to create key performance indication (KPI) } \\
\text { matrix. }\end{array}$ \\
\hline $\begin{array}{l}\text { Sharing the analysis } \\
\text { results and the routes }\end{array}$ & $\begin{array}{l}\text { Dashboard should be able to provide the user a visual } \\
\text { understanding of the analysis and the routes on the map } \\
\text { for a particular user-defined day. }\end{array}$ \\
\hline
\end{tabular}

\subsubsection{Non-Functional Requirements}

The non-functional requirements included technical, operational, and transitional aspects of the project. Table 2 lists all the non-functional requirements.

Table 2. System Design Non-Functional Requirements

\begin{tabular}{|l|l|}
\hline Requirement & Description \\
\hline Operating System & $\begin{array}{l}\text { Windows 7, 64-bit operating system, 8 GB RAM, and } \\
3.00 \mathrm{GHz} \text { processor speed }\end{array}$ \\
\hline Esri ArcMAP 10.3 & $\begin{array}{l}\text { To hold the enterprise geodatabase, format and verify } \\
\text { data, modify the locations of the customers }\end{array}$ \\
\hline $\begin{array}{l}\text { Network Analyst } \\
\text { extension }\end{array}$ & To create the routes. \\
\hline Enterprise Geodatabase & To store all the input and output data. \\
\hline
\end{tabular}




\begin{tabular}{|l|l|}
\hline Requirement & Description \\
\hline Esri ArcGIS Online & To create the web map and link the tool with dashboard. \\
\hline $\begin{array}{l}\text { Esri Operations } \\
\text { Dashboard }\end{array}$ & $\begin{array}{l}\text { Monitor activities and events, track field workforce, and } \\
\text { assess the status and performance of daily operations. It } \\
\text { also adds a visual aspect to the result making them easy } \\
\text { to understand. }\end{array}$ \\
\hline
\end{tabular}

A Windows 7 operating system was the preferred technical requirement, although a higher version of Windows would provide higher efficiency for the result generation time. The hardware specifications for this project included a 64-bit operating system with $8 \mathrm{~GB}$ of RAM and a $3.00 \mathrm{GHz}$ processor speed. A higher RAM size was desirable for better system performance as the project involved complex data extraction and route optimization algorithms. Technical requirements included creation of the enterprise geodatabase to hold the feature classes and operational tables.

\subsection{System Design}

The ROAR consisted of four key components: data extraction and preparation; routing tool; analysis and dashboard; and integration, testing, and documentation. Figure 3-1 illustrates the project design and connectivity of the components.

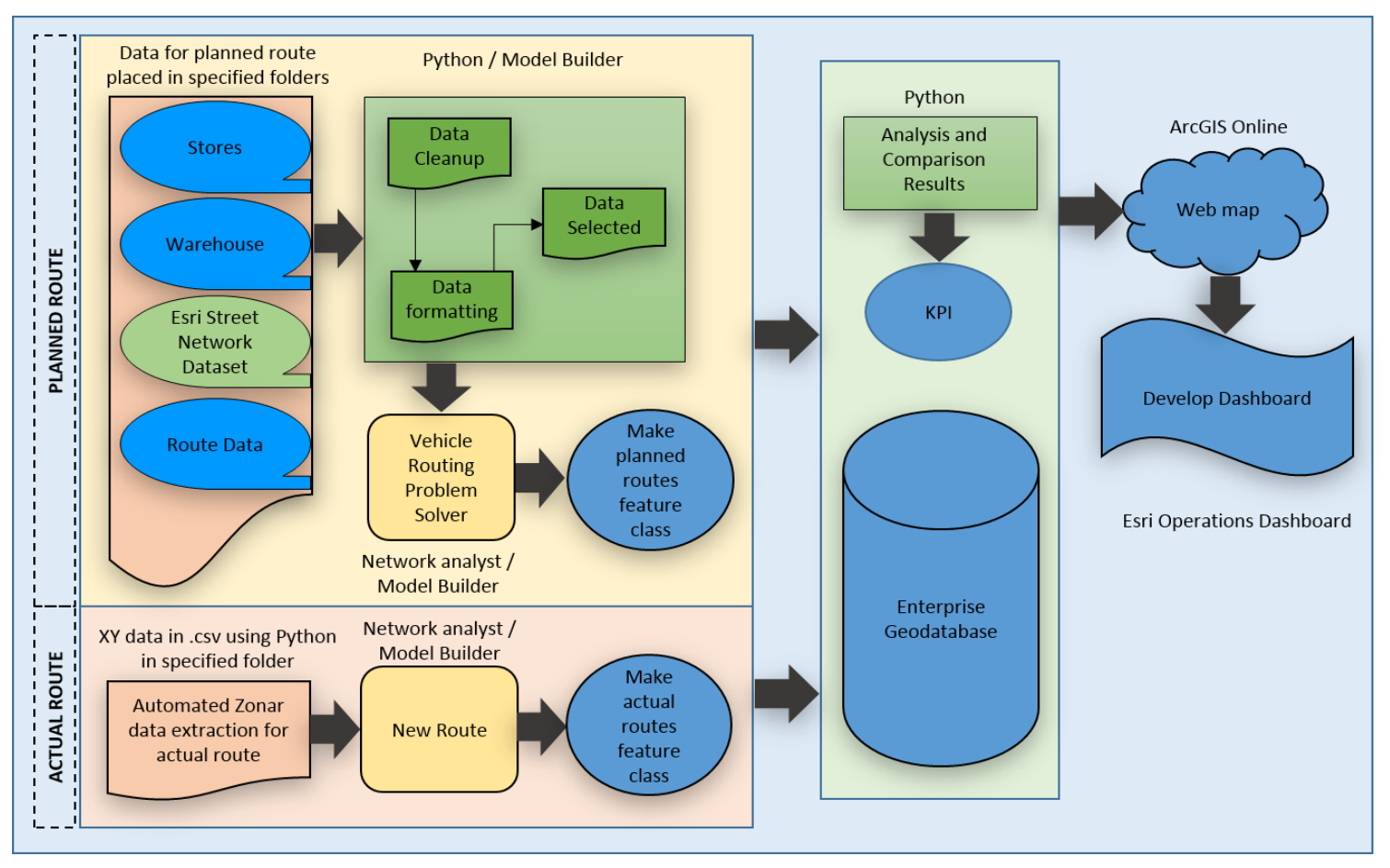

Figure 3-1: System Design for ROAR 
The first component of this project focused on data acquisition. For the actual routes, data existed in the third party fleet management systems in the form of $\mathrm{xml}$ web services. Different built-in functionalities could download the data reports, collected from the GPS trackers embedded on the distribution trucks. An automated Python tool was required to extract the XY data from the web services in comma separated values (CSV) format. These CSV files were the only required data for actual routes. No cleanup process was required for these data, as they were collected from an existing live system. Recommended routes generation needed more data in comparison to the approximation of actual routes, and these data included customer and warehouse locations. Along with the customers and warehouse locations, data for service days, service time, time windows, and volume were provided in different Excel sheets. Other datasets required were the Esri Street Network dataset and truck information. The data were cleaned to ensure they contained no errors when input to the routing tool. The geodatabase was created to hold all the datasets in one place with the output results.

The second component involved the creation of two routing tools used to create approximate actual routes and recommended routes. After GPS data were downloaded to CSV files, the actual route was generated by connecting the GPS points. Recommended routes were created using a vehicle routing problem (VRP) algorithm.

The third component was the creation of an analysis script that generated the key performance indicators (KPI). Once these were generated, the focus of the project moved to sharing the results. After selection of a particular date, analysis results between the actual and recommended route for a selected route ID would be displayed and shared with multiple departments.

The fourth component included the integration of the previous components. The tool was tested with an extensive use case. The tool was delivered to the client with a document explaining the installation steps.

\subsection{Project Plan}

The development of the project plan helped keep the project on track considering different parameters such as scope, time, requirements, training, risks, and deliverables. Milestones and a timeline were created to record the progress of the project life cycle. The project plan was created using the Agile project management approach, which included frequent communication with the client. This iterative process allowed focusing on one component at a time until satisfactory results were produced. There were five key phases to the project: planning, research, data review, analysis, and project delivery.

The project planning phase started with initial client discussions to gain a thorough understanding of the problem. Although solutions were discussed, deliverables and requirements were the focus points. Using the Agile project management approach, requirements were discussed at the beginning of every phase. Other necessary discussion topics included the business impact of not having optimized routes, since the results would directly influence the profits. The project scope was defined and a reasonable 
schedule was planned. Enough time was given for completion of each component in order to validate the outputs. Every component was verified with user acceptance test.

Extensive and thorough research was conducted, including the investigation of similar case studies, using GIS and other technologies to generate optimized routes. The literature review in Chapter 2 helped to decide the right algorithm for this project. Vehicle Routing Problem (VRP) solver was the right choice in order to include constraints like service time, time window, volume, and vehicle capacity. The literature reviews on dashboard for this type of project were limited. Irrespective of these challenges, Esri had substantial documentation available on the capabilities of the network analyst and operations dashboard. One common element between many research documents was the outcome: a large cost savings due to a decrease in miles driven, travel time, overtime, and maintaining time windows and service times.

The data collection involved the datasets needed to create the routing tools. All datasets obtained were thoroughly reviewed for quality and consistency. The locations of the customers and the warehouse on the recommended routes needed several edits, including removal of duplicate customers and those beyond the study area. A visual inspection was done to ensure all points were within the study area. Further data involved the time window, service time, and demand for the Red Bull product. The actual route data were extracted after understanding the built-in functionalities of the Zonar system. These data were integral for the analysis since multiple attribute fields were used during the route optimization calculations. Additional data obtained during multiple client meetings included truck capacity, driver work hours, analysis parameters, and dashboard design.

The project implementation phase started with creating one enterprise geodatabase. Data formatting was done for the recommended route data and an automation script was created for extracting third party data for actual route data. All information was fed to the VRP model and route solver parameters were adjusted to meet the client's criteria. The store points in this study were divided into smaller groups for more efficient processing during analysis. The VRP solver layer was created and configured with the start and finish points. Locations were loaded into the VRP solver and extra settings were configured on the layers. The Network Analyst solve tool was executed to model routes based on defined settings and criteria. Generated routes were published on ArcGIS online and a web map was created. A Python script was created to perform the analysis and to generate the key performance indicators (KPI) and dashboard.

In the last phase, the client received a detailed instruction document of the ROAR tool. This document also provided details on the implemented folder system. The client referred to this document to perform changes while doing the installation. Extra data, tables, and documents relevant to the project were included as a digital deliverable on a USB flash drive. 


\subsection{Summary}

System design and project planning were discussed in this chapter. The chapter outlined the problem addressed, functional and non-functional requirements, and identified the proposed solution. These elements were the backbone of this project and helped achieve the needed results. Implementation steps performed for each component are discussed in upcoming chapters. 


\section{Chapter 4 - Database Design}

An ArcGIS enterprise geodatabase was created to store the required data and to provide the vehicle routing problem (VRP) solution. The geodatabase consisted of the customer and warehouse details. The geodatabase also included recommended routes, approximate actual routes, and the customers served by both. This chapter contains the design and implementation of the geodatabase and the data that were stored in it. Section 4.1 addresses the conceptual data model. Section 4.2 describes the logical data model. Section 4.3 and Section 4.4 examine information on the data sources used in this project. Section 4.5 shows how the data were prepared. Finally, Section 4.6 concludes this chapter with a summary.

\subsection{Conceptual Data Model}

A conceptualization of the process utilized for developing a project generates successful outcome for a client-based project. This allows the project developer to formulate plans and understand important details. A conceptual model was designed according to the data requirements to solve the problem (Figure 4-1).

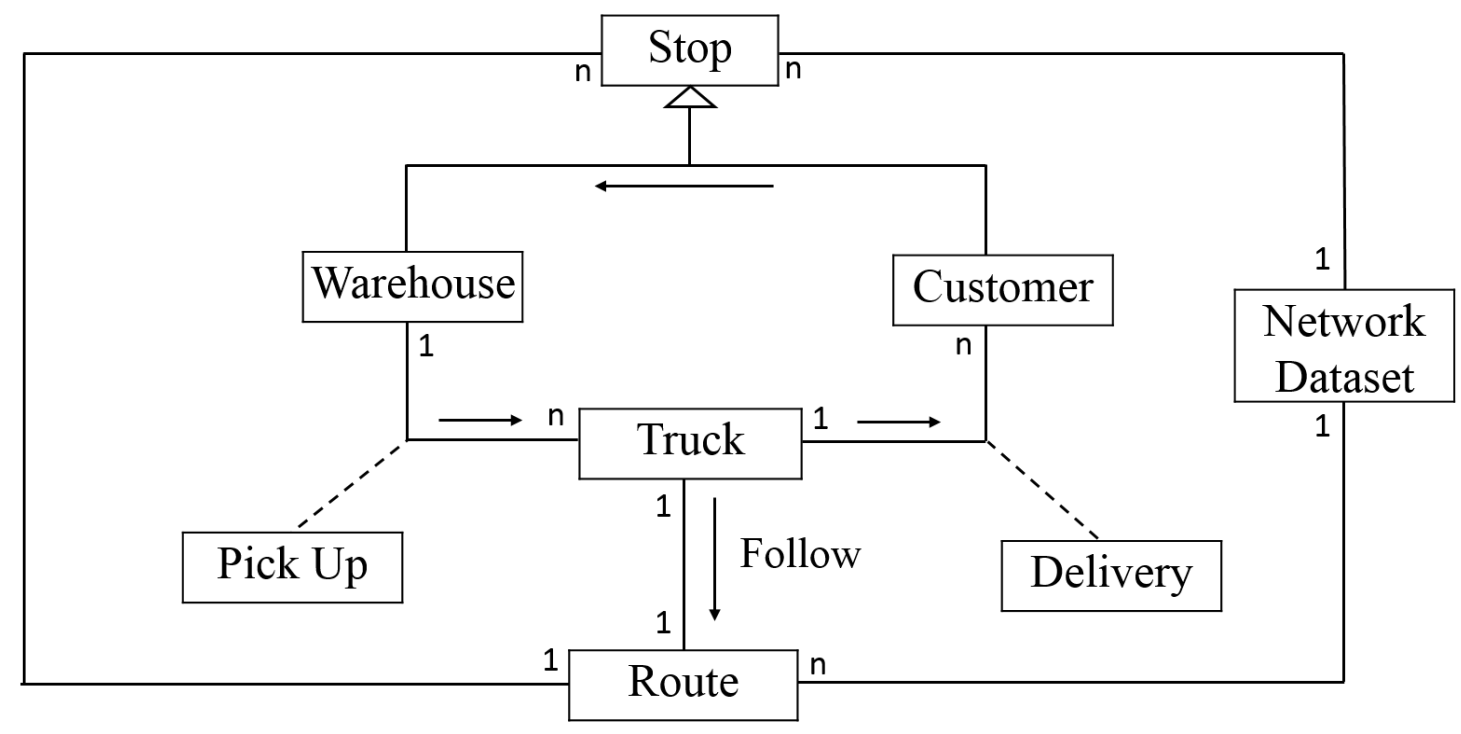

Figure 4-1: Conceptual Model

Route Optimization and Analysis Register (ROAR) was a routing project for distribution of the Red Bull products from the warehouse to all the customers within a service area. ROAR was then used on all the predefined service areas. The four primary classes included Truck, Route, Location, and Street Network. There is a direct relationship between the truck and the route. Locations have two subclasses: Warehouse and Customer. The truck started the process by picking up the product from the warehouse location, traveled along the route, proceeded to a series of customer locations with specific deliveries, and returned to the warehouse. One warehouse was associated 
with multiple trucks; however, a truck was associated with a particular set of stores. These routes were linked to the Street Network dataset from Esri.

\subsection{Logical Data Model}

To meet the needs of the project, a conceptual model was designed as a high-level interpretation of the requirements. On the other hand, a logical model is a representation of the structure for the geodatabase design. The logical model detailed the data requirements to solve the problem that ROAR addressed in the conceptual model. Figure 4-2 describes the logical model for the enterprise geodatabase.

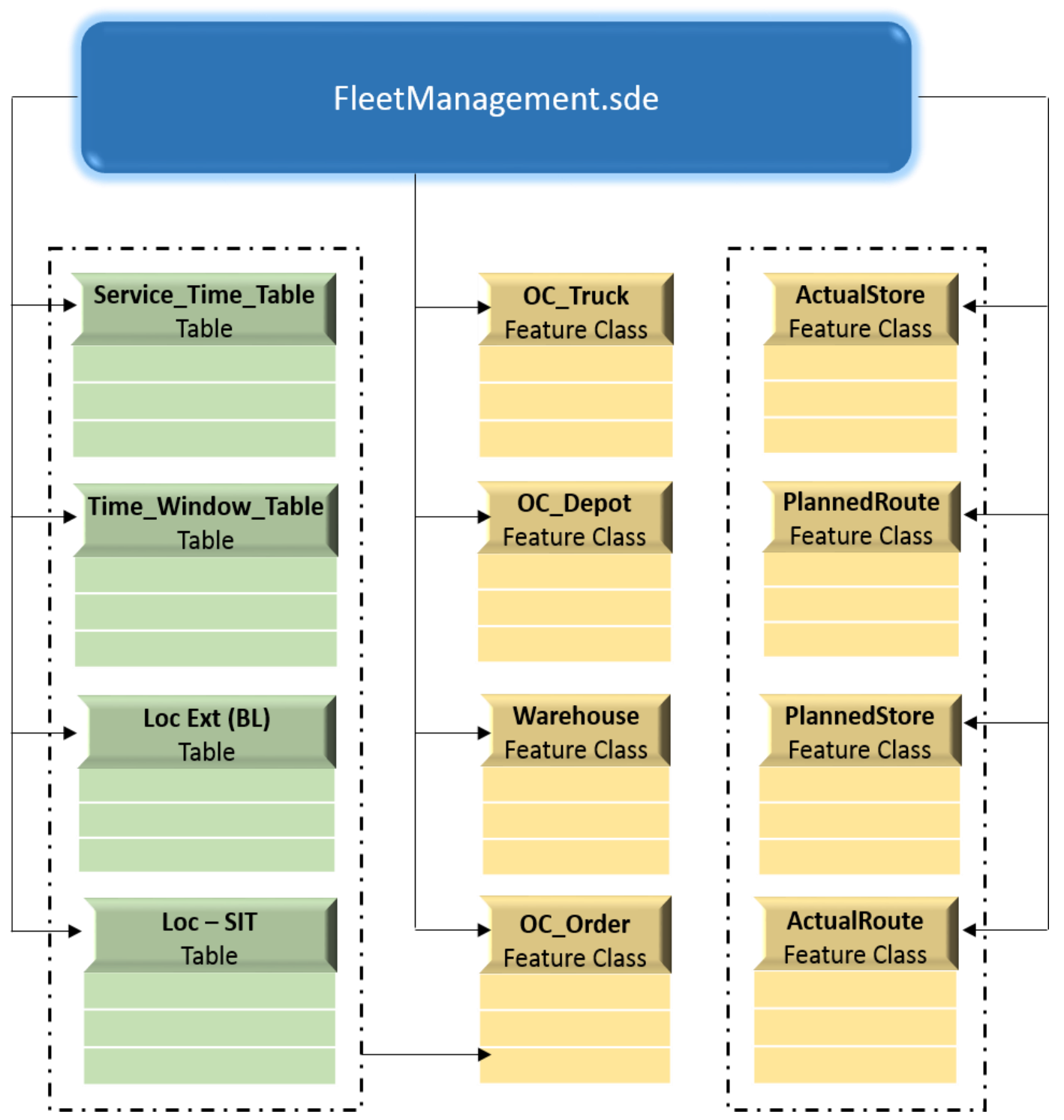

Figure 4-2: Logical Data Model for the File Geodatabase

Feature tables and feature classes were associated with the enterprise geodatabase. The table Loc-SIT stored the location details of the customer. The table Loc Ext (BL) 
contained the volume matrix. The table Time_Window_Table detailed the allowed time of day when a particular type of customer could be served. The table Service_Time_Table held the average service time for each customer. All the input data for generating the recommended routes can be found in Appendix A. Feature class OC_Order was created by combining the four input tables to hold the customer details. Along with the customer feature class, the OC_Depot feature class containing the warehouse details and the $O C_{-}$Truck table holding the path and truck details were the inputs for the VRP solver. The feature class RecommendedRoute detailed the recommended distance and recommended time, along with important attributes such as the number of customers to be served, delivery volume, service time, and travel time. The feature class ActualRoute detailed the same attributes like that of the RecommendedRoute feature class, but these details were from the actual truck routes created after the truck returned to the warehouse. It also held the attributes from the recommended routes in order to keep all the details in the same feature class, making the comparison between the two routes efficient.

\subsection{Data Sources}

All the data were collected from two sources. Red Bull had its own GIS team who provided the major sets of data: operations data in Excel sheets, warehouse, and truck details. The customer feature class was created by using ArcGIS tools. The Zonar fleet management system supported the client and was responsible for storing the data from the actual route using GPS devices. The details for each actual route were generated with a call to an XML web service in the Zonar system and were extracted using a Python program developed in this project.

\subsection{Data Collection Methods}

The data collection method was not very straightforward for this project. After intensive research on the data requirements, details of the required variables were requested from the client. Based on the data requirements, the digital data were shared using confidential authentication. Username, user ID, and password to the Zonar system were provided in order to access and extract the GPS data.

\subsection{Data Scrubbing and Loading}

Cleaning and formatting of the data provided by the client was important to avoid errors while running the ArcGIS tools. The first set of data provided by Red Bull's GIS team was in four Excel spreadsheets. The Location spreadsheet had the customer details, including customer ID, name, latitude, longitude, route ID, customer type, service time pattern, time window pattern, and address. These data needed a considerable review and cleaning. The list had 1,951 customers, out of which 417 customers were missing the latitude and longitude values. The remaining 1,534 customers were loaded to ArcMap using the "Add XY Layer" tool. The layer was converted into the customer feature class, using the ArcGIS "Export Data" tool. Customers with missing location fields were geocoded separately with the address field. Geocoding was not successful for 19 customers as ArcGIS geocoder either could not find the address or found duplicate values. The latitude and longitude details for these 19 customers were found manually 
from internet and entered in the Excel spreadsheet. The customers with missing latitude and longitude values were merged with the created customer feature class.

\subsection{Summary}

This chapter provided details on both the conceptual and logical models of the project database. The project data classes and attributes, as well as their relationship to each other within the geodatabase, were described in the conceptual model section. The logical model section highlighted the process of physical database construction based on the conceptual model design. The data source and data collection methods sections discussed both where and how the necessary data were obtained. The final section described the integrity of the obtained data and the approaches taken to assure the data met the quality standards necessary to perform analysis. 


\section{Chapter 5 - Implementation}

Route Optimization and Analysis Register (ROAR) was developed in phases that included cleaning and formatting of data, developing two sets of routes, designing key performance indicators (KPI) for analysis, and sharing of results. This chapter outlines the steps taken in creating each phase of ROAR. Section 5.1 discusses the creation of the feature classes in the enterprise geodatabase. Section 5.2 focuses on the creation of two sets of routes: recommended and actual. Section 5.3 discusses the development of the KPIs. The components of Sections 5.1, 5.2, and 5.3 of this chapter were developed using two Python scripts: RecommendedRoute.py and ActualRoute.py. Section 5.4 explains the configuration process for sharing of the KPI results. Section 5.5 concludes the chapter.

\subsection{Creating Feature Classes in the Geodatabase}

A Python script was developed to create four empty feature classes in the enterprise geodatabase in order to store the output routes and customers (Appendix E). These feature classes were ActualRoute, RecommendedRoute, ActualStore, and RecommendedStore. The path for the geodatabase was defined as the workspace. WGS_1984_Web_Mercator_Auxiliary_Sphere was selected as the spatial reference for all feature classes. Feature classes were registered as versioned in order to avoid any changes to the main version of these feature classes, a functionality that is supported for feature classes in an enterprise geodatabase.

After the feature classes were created, an appropriate design of the schema was required in order to store the results from ROAR. Therefore, new fields were added to the recommended feature class. Some of the important fields in the recommended route feature class were name, date, total time, service time, travel time, distance travelled, and number of customers. The actual route feature class contained similar fields: time, distance, and customer count fields with the suitable name and date. The actual route feature class also stored the attributes from the recommended routes. This allows ROAR to perform the analysis from the same feature class. The actual and the recommended store feature classes had fields to store the names of the routes and the dates.

\subsection{Recommended Route}

The customer feature class was created during the data-cleaning phase. The feature class was joined to the operational tables to add the customer service details. The operational table included volume matrix, service time, and time window. This section also describes how the removal of duplicate customers was handled. After the data were processed, recommended routes were created using the vehicle routing problem (VRP) solver within the ArcGIS Network Analyst extension. The script developed to create recommended routes is available in Appendix $\mathrm{F}$ for referral.

\subsubsection{Data Formatting}

The volume matrix table provided by the client contained delivery volume, service weeks, and delivery days for all customers. The volume matrix table was joined with the 
customer feature class based on the location IDs. The service time table contained 16 customer types and included details of allotted time to service each type of customer. The service time table was joined to the customer feature class based on the customer type in order to store the service time in the customer feature class. Another join was executed to include the delivery time window in the customer feature class.

The script included formatting service week, delivery day, and volume. Three fields were added to the customer feature class: ServiceDay, ServiceWK, and Volume. The week field included space representing inactive weeks. This was problematic since leading spaces were ignore by the geoprocessing tools. The spaces were replaced by underscore ('_'). For example, if a customer had weeks 1 and 3 as service weeks and weeks 2 and 4 as inactive weeks, the original table presented it as " $\mathrm{X} \mathrm{X}$ " ("X $<$ space $>X<$ space $>$ "). The script formatted the field and replaced empty spaces with underscores. The results were stored in tunknownhe ServiceWK field. Table 3 lists the possible conversions from space to underscore for the inactive service weeks.

Table 3. Service Week Formatting

\begin{tabular}{|c|c|c|}
\hline Service Week & Original & Generated Output \\
\hline 1 & $\mathrm{X}$ & $X_{---}$ \\
\hline 2 & $X$ & $\mathrm{X}_{--}$ \\
\hline 3 & $X$ & ${ }_{-} \mathrm{X}_{-}$ \\
\hline 4 & $X$ & $-\ldots X$ \\
\hline $1 \& 2$ & $\mathrm{XX}$ & $\mathrm{XX}_{--}$ \\
\hline $1 \& 3$ & $\mathrm{XX}$ & X_X_ \\
\hline $1 \& 4$ & $\mathrm{X} \quad \mathrm{X}$ & $X_{-} X_{1}$ \\
\hline $2 \& 3$ & $\mathrm{XX}$ & XX_ \\
\hline $2 \& 4$ & $\mathrm{XX}$ & _X_X \\
\hline $3 \& 4$ & $\mathrm{XX}$ & _- XX \\
\hline All four weeks & XXXX & $\mathrm{XXXX}$ \\
\hline
\end{tabular}

Formatting for service day was also required. Boolean numbers were used. For each day of the week that was considered active, ' 1 ' was assigned. ' 0 ' was assigned if the day was inactive. The Python script calculated the ServiceDay field using this logic. The original values and the generated output are shown in Table 4. 


\section{Table 4. Service Day Formatting}

\begin{tabular}{|l|l|l|}
\hline Service Day & Original & Generated output \\
\hline Monday & M & 000001 \\
\hline Tuesday & T & 000010 \\
\hline Wednesday & W & 000100 \\
\hline Thursday & R & 001000 \\
\hline Friday & F & 010000 \\
\hline Saturday & S & 100000 \\
\hline Monday \& Tuesday & MT & 000011 \\
\hline Monday \& Wednesday & MW & 000101 \\
\hline Monday \& Thursday & MR & 001001 \\
\hline Monday \& Friday & MF & 010001 \\
\hline Monday \& Saturday & MS & 100001 \\
\hline Tuesday \& Wednesday & TW & 000110 \\
\hline Tuesday \& Thursday & TR & 001010 \\
\hline Tuesday \& Friday & TF & 010010 \\
\hline Tuesday \& Saturday & TS & 100010 \\
\hline Wednesday \& Thursday & WR & 001100 \\
\hline Wednesday \& Friday & WF & 010100 \\
\hline Wednesday \& Saturday & WS & 100100 \\
\hline Thursday \& Friday & RF & 011000 \\
\hline Thursday \& Saturday & RS & 101000 \\
\hline Friday \& Saturday & FS & 110000 \\
\hline Monday \& Wednesday-Friday & MWF & 010101 \\
\hline Monday, Tuesday, Wednesday, Thursday, \& Friday & MTWRF & 011111 \\
\hline Tuesday, Wednesday, Thursday, Friday, \&Saturday & TWRFS & 111110 \\
\hline & & \\
\hline
\end{tabular}

The volume matrix provided by the client included product volume for each customer. Product volumes were presented in three fields: Size 1, Size 2, and Size 3. The product volume of interest was listed in field size 1 if the service week pattern was classified as every week of the month. Field size 2 stored the product volume for biweekly pattern, and size 3 for the monthly pattern. For example, for customer who had a service week pattern of every week (XXXX), the value considered for the product volume will be extracted from the field 'Size 1', ignoring the values in Size 2 and Size 3. Table 5 provides an example of each service week pattern and the field from which the product volume for each customer was extracted. Duplicate customer entries were discovered in the original data, which was problematic because ROAR required unique customer entries for generating the routes. A new field, DuplicateCheck, was created to identify the duplicate customer entries. For example, if a customer was found in the customer feature class for the first time, a value of " 1 " was assigned in field DuplicateCheck. The second time a customer was found in the feature class, a value of 
" 2 " was assigned, the third was " 3 ", and so on. The duplicate records were removed from the customer feature class.

Table 5. Volume Based on the Service Week Pattern

\begin{tabular}{|c|c|}
\hline Service week Pattern & Volume \\
\hline$X X X X$ & Size 1 \\
\hline$X X_{-}$ & Size 2 \\
\hline$X \_X_{-}$ & Size 2 \\
\hline$X_{-} X$ & Size 2 \\
\hline XX_ & Size 2 \\
\hline _X_X & Size 2 \\
\hline _-_XX & Size 2 \\
\hline$x_{-}-{ }_{2}$ & Size 3 \\
\hline$x_{-}$ & Size 3 \\
\hline$\ldots$ & Size 3 \\
\hline 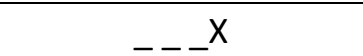 & Size 3 \\
\hline
\end{tabular}

\subsubsection{Route Generation: VRP}

To generate the recommended routes, the required data were formatted and cleaned, as discussed in the Section 5.2.1. The developed Python script used the system date to identify the customers to be serviced from the customer feature class based on service week and service day. For example, if the script was executed on a Tuesday in the third week of the month, customers that had the corresponding service day and service week were identified. This process created a list of customers to be served on the particular day. The VRP solver is one of the routing algorithms in the ArcGIS Network Analyst extension. The VRP solver workflow is illustrated in Figure 5-1. 


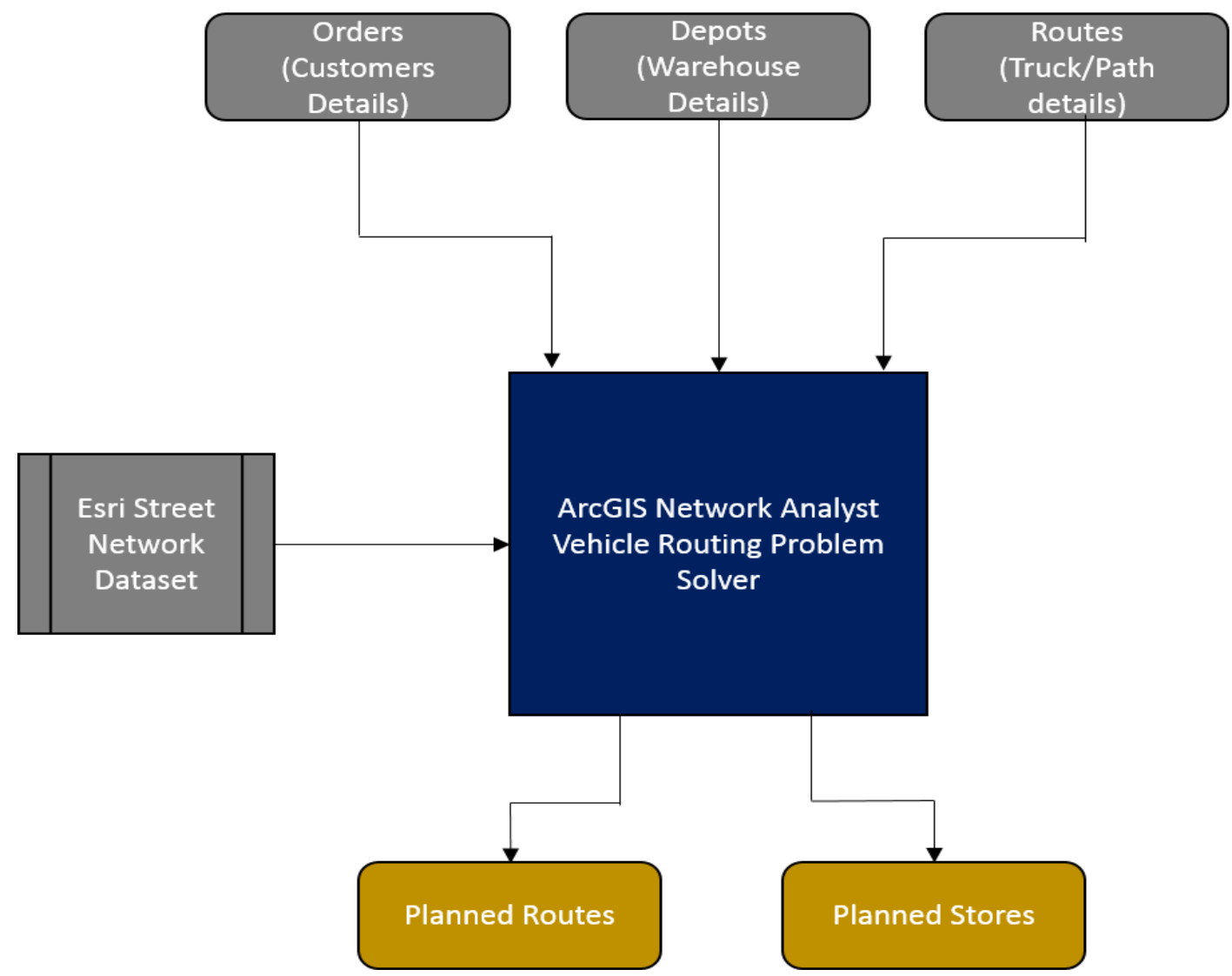

Figure 5-1: Vehicle Routing Problem Solver Detailed Workflow

The function of the VRP solver is to deliver orders and reduce overall operating time and costs for traveling salesmen. An empty VRP layer was created for the Esri Street Network dataset. Output route units were set as miles and minutes. In addition, restrictions were added that forced distribution trucks to avoid non-navigable streets. The VRP layer contained 13 sublayers by default, out of which three were used for this project: Depots, Orders, and Routes. The Depots sublayer was meant to store the warehouse details, while the Orders sublayer was for the customer details. The Routes sublayer was populated with the vehicle, routes, and driver characteristics.

Figure 5-2 displays the Orange County warehouse and the associated customer locations. The warehouse location was loaded into the Depots sublayer using the script with the corresponding attributes, such as operating hours, name, and description. 


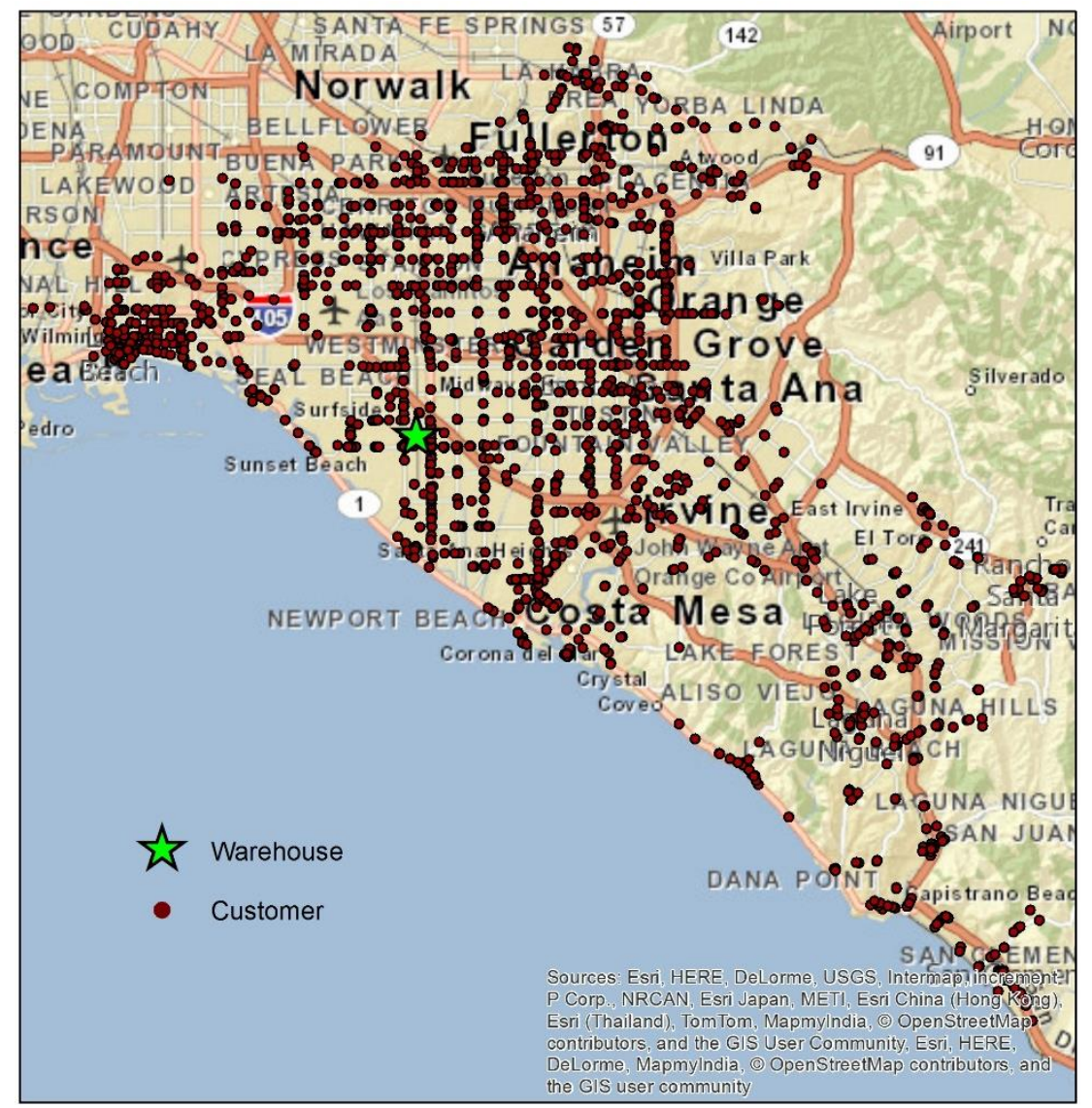

Figure 5-2: Warehouse and Customer Locations in Orange County

The script would add selected customers to be served in the Orders sublayer. On average, 300 customers were served daily. The script then grouped the selected customers based on the route IDs stored in the customer feature class. The script looped through the customer groups and loaded the Orders sublayer with the required group of customers. Customer attributes such as allowed service time, time windows for the customers, and delivery volume were added to the Orders sublayer. These attributes were retrieved from the customer feature class. There were default values provided by the client, in case details were missing for any customer in the feature class.

The client confirmed that a truck could go to only one zone. In addition, a truck can serve up to 25 customers and its maximum capacity is 350 cases. The script found these attirbute values either in the truck feature table or coded as default values and loaded the into the Routes sublayer. Utilizing the three sublayers used in this project, the script generated the optimized routes by the VRP solver.

The last part of the script generated the recommended routes and the customers served for each route. The generated routes had attributes such as travel time, service time, total time, distance travelled, and count of customers visited. This part of the script combined all the customers as one single multipoint feature in order to make it convenient for the users to see all the locations at one single click. Finally, all the routes 
and the customers were stored in the Recommended Route feature class and Recommended Store feature class, respectively, in the enterprise geodatabase.

\subsection{Actual Route}

Actual route information for the distribution trucks was recorded daily and stored on a Zonar Fleet Management system, available to the client as XML web services. A Python script was developed to work with the actual route data. The script logged into the third party system using valid credentials. The script developed to create recommended routes is available in Appendix $\mathrm{G}$ for referral.

\subsubsection{Data Extraction}

The script created a start and end time which included the 24 hours of the script execution day. Figure 5-3 displays the flow chart representing the functions executed by the script. Each truck was selected from the Zonar Fleet Management system and verified if it was associated with Orange County. If the truck was found to be associated with Orange County, then the script extracted the GPS points between the start and the end time. The data were in the form of sequential points with latitude and longitude collected by the GPS unit on the truck.

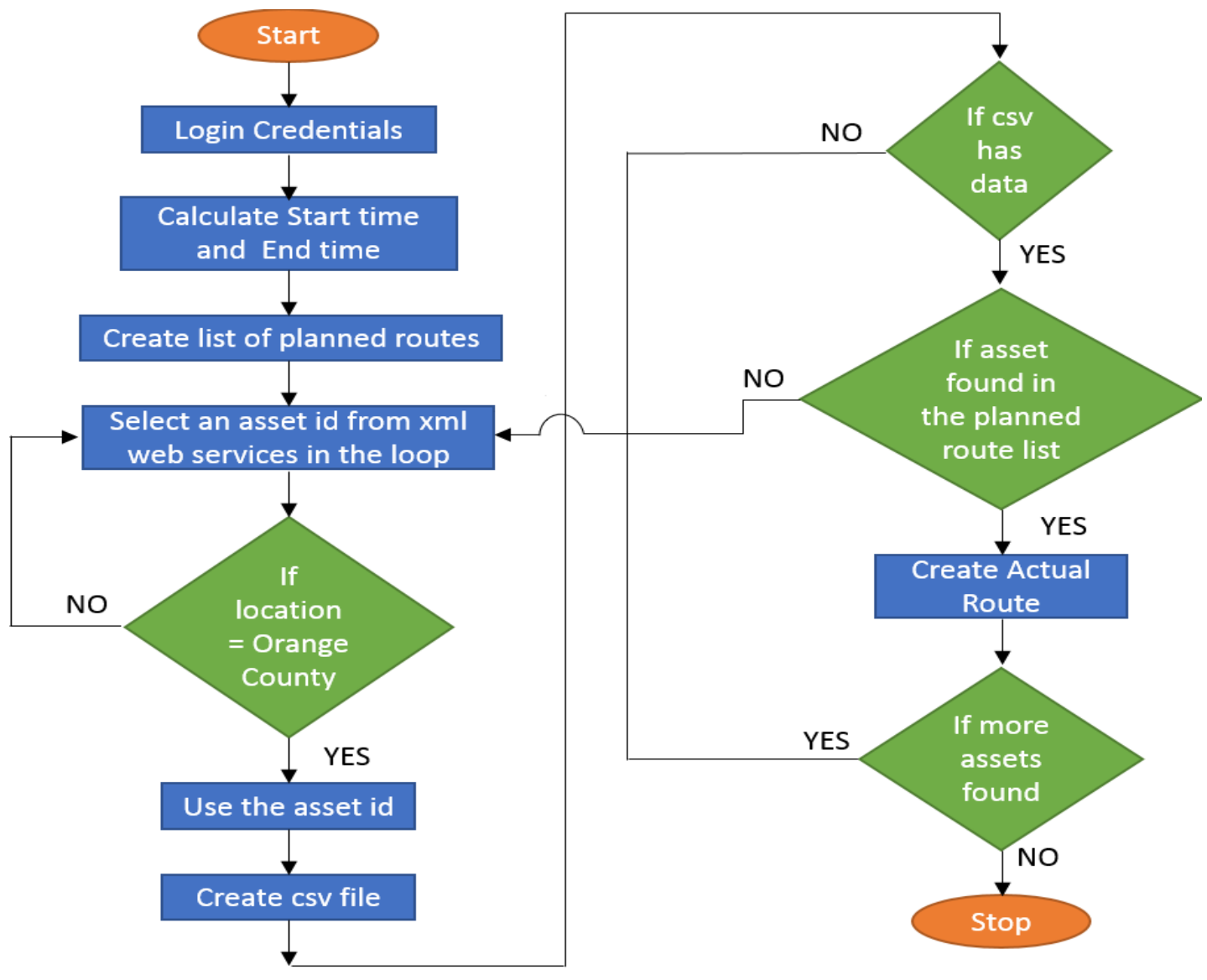

Figure 5-3: Flowchart for Data Extraction from Zonar Web Services. 
The extracted data were stored in a CSV file in the project folder. This CSV file was used to create the approximate actual route. This process was repeated for all the trucks available from the Zonar web services. The resulting CSV file was compared to the recommended routes in order to ensure there was a recommended route corresponding to an actual route truck. The purpose of this comparison was to verify if the CSV file extracted from the third party system needed to be rejected. If a recommended route was not found for a CSV, the file was removed from the analysis. In addition, any CSV file which contained no GPS data was rejected, as the distribution truck was not active that day. These rejection criteria were included in order to avoid unwanted run time of the Python scripts.

\subsubsection{Route Identification}

The script used the GPS points, converted them into a feature layer, and then converted the layer into a feature class. It was problematic to convert the points into a feature class without the right geographic coordinate system. The research helped in understanding of the correct coordinate system that was required.

The script connected all the GPS points in sequence using the "Point to Line" geoprocessing tool in ArcGIS and generated the approximate actual route. These routes were the actual paths that the trucks took from the warehouse delivering products to customers. Since these routes were not created using Network Analyst, route attributes such as time and distance were not generated. The script found the recorded time of the first point and the last point from the CSV file and calculated the total time. The total distance travelled was extracted from the distance stored for the last GPS point. The time spent between each GPS "power on" and "power off" log was considered as the service time for the respective customers. However, a 'power on' and a 'power off' were not necessarily associated with a customer stop. Therefore, based on discussions with client it was assumed if the time spent was more than 90 seconds then a customer was being served. All the individual calculated service times were added as the total service time for the route. The total travel time for the actual route was found by subtracting the total service time from the total time. During the extraction of the service time, the script kept a count of customers being served and stored the actual store count.

The script added the generated actual route with its attributes and stored the route in the ActualRoute feature class. The script also combined all the customers as one single multipoint feature in order to make it convenient for the user interface to display all the locations with a single click. The customers were added to the ActualStore feature class in the enterprise geodatabase.

\subsection{Key Performance Indicators}

One of the goals for this project was to create a tool that could compare recommended and approximate actual routes. To do so, ROAR used three key performance indicators (KPI): distance, total time, and customer count. Analysis was done within the actual route script. The route attributes of the recommended route feature class from the enterprise geodatabase were extracted, including fields such as recommended distance, 
recommended service time, recommended travel time, recommended total time, and recommended customer count. These attributes were stored in the ActualRoute feature class. The script subtracted the actual route attributes from the recommended route attributes and calculated the KPIs. Results from the calculated KPIs were stored in the Actual Route feature class.

\subsection{Dashboard Configuration}

The routes were generated and KPIs were calculated using Python programming. The final requirement of the project was to provide an interface that can display the KPI results. Based on the client's requirements, it was decided that Esri's Operations Dashboard would be used. According to the requirements, the dashboard had to be updated on a daily basis with the recommended and approximate actual routes. In order to automate the process of routing and calculating KPIs, the developed scripts were added on Windows task scheduler. Figure 5-4 display the steps used to schedule the recommended route script.

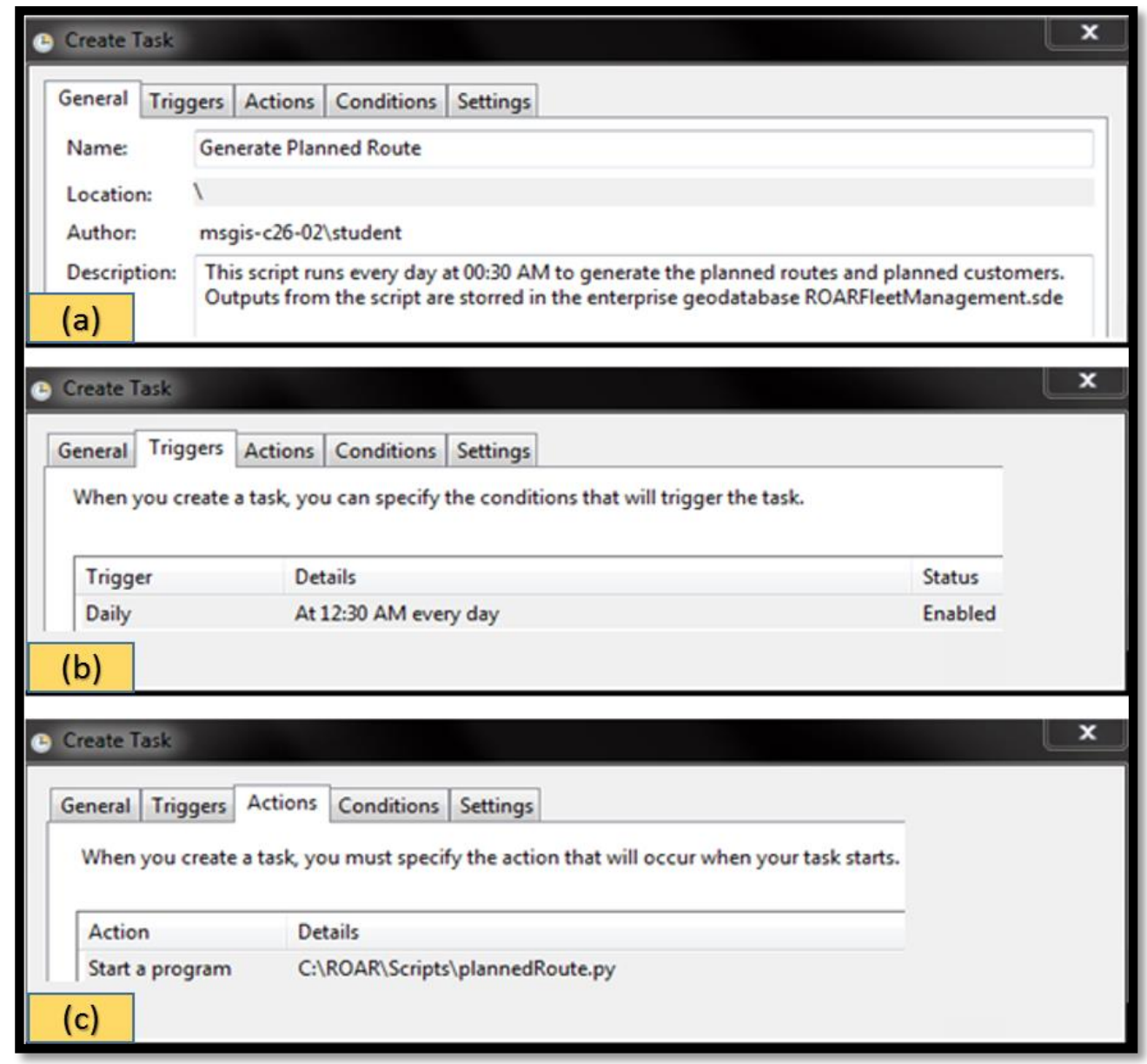

Figure 5-4: Scheduling of Recommended Routes Generation. General (a), Trigger (b), and Action (c) 
In the Windows task scheduler a new task was created. This task was configured and edited with a name and a description as shown in the Figure 5-4a. The trigger settings were edited in order for the task to run every day at 12:30 AM. It was required to set the status of the task as active, illustrated in Figure 5-4b. Finally, the script for generating the actual routes was attached to the task shown in Figure 5-4c. The same process was followed to schedule the actual route identification script at 11:30 PM every night.

All feature classes and feature tables stored in the enterprise geodatabase were published to an ArcGIS for Server. These published feature services were used to create a web map on ArcGIS Online. The web map was configured to customize the pop-ups and add bookmarks. These configurations were retained in the dashboard. Operations Dashboard is a downloadable Esri product that monitors activities and tracks performance of events on a daily basis. For this project, the users required an ArcGIS Online organization account to access the results on the dashboard. A new Multidisplay operation view was created in the dashboard (Figure 5-5).

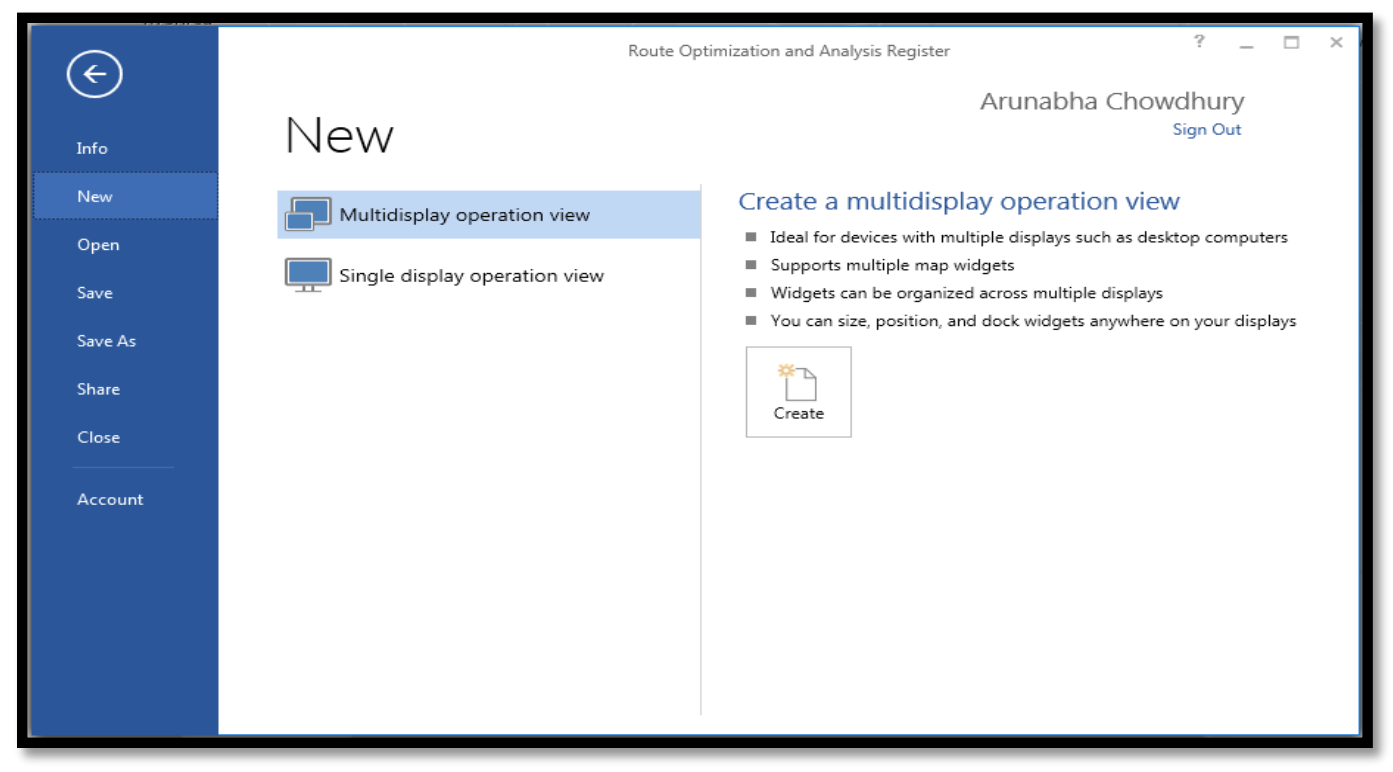

Figure 5-5: Create New Operations View

The created operations view was empty and was not associated with any map. The first widget used was the map widget. The web map URL was linked to the dashboard using the New Map widget. As displayed in Figure 5-6, all the feature layers from the web map were marked as checked. A title was provided and the widget was saved. A Legend widget was also added in order to simplify the map reading for a user. 
Settings

Title: ROUTE OPTIMIZATION AND ANALYSIS REGISTER (ROAR)

Data Sources

Capabilities

Check the layers to be included as data sources for other widgets. Indicate whether each layer contains dynamic data and whether features can be selected.

Data Sources Settings
$\nabla \therefore$ Warehouse
$\checkmark$ 戋 Actual Stores
$\checkmark$ 屡 Planned Stores
$\nabla \therefore$ Customers
$\checkmark \bowtie$ Planned Route
$\checkmark$ Actual Route

\section{Static: No automatic update}

$\checkmark$ Selectable

Filters:

Remove

Figure 5-6: Configuration of the Map Widget.

Four List widgets were created to display the features from the ActualRoute, RecommendedRoute, ActualStore, and RecommendedStore feature layers. Data Source was selected as the required feature layer from the drop down list and a title was added for each of the widgets (Figure 5-7). All widgets allowed filtering within the list in order to find the route based on the route ID or the date. A separate section was provided using a Description widget helping a user know how the filter would work. List widgets would be sorted based on the date and a maximum of 2000 features would be displayed on the dashboard. Date and name of the route would be visible on the dashboard. On selecting a route, zoom-to and highlight actions would be allowed. 


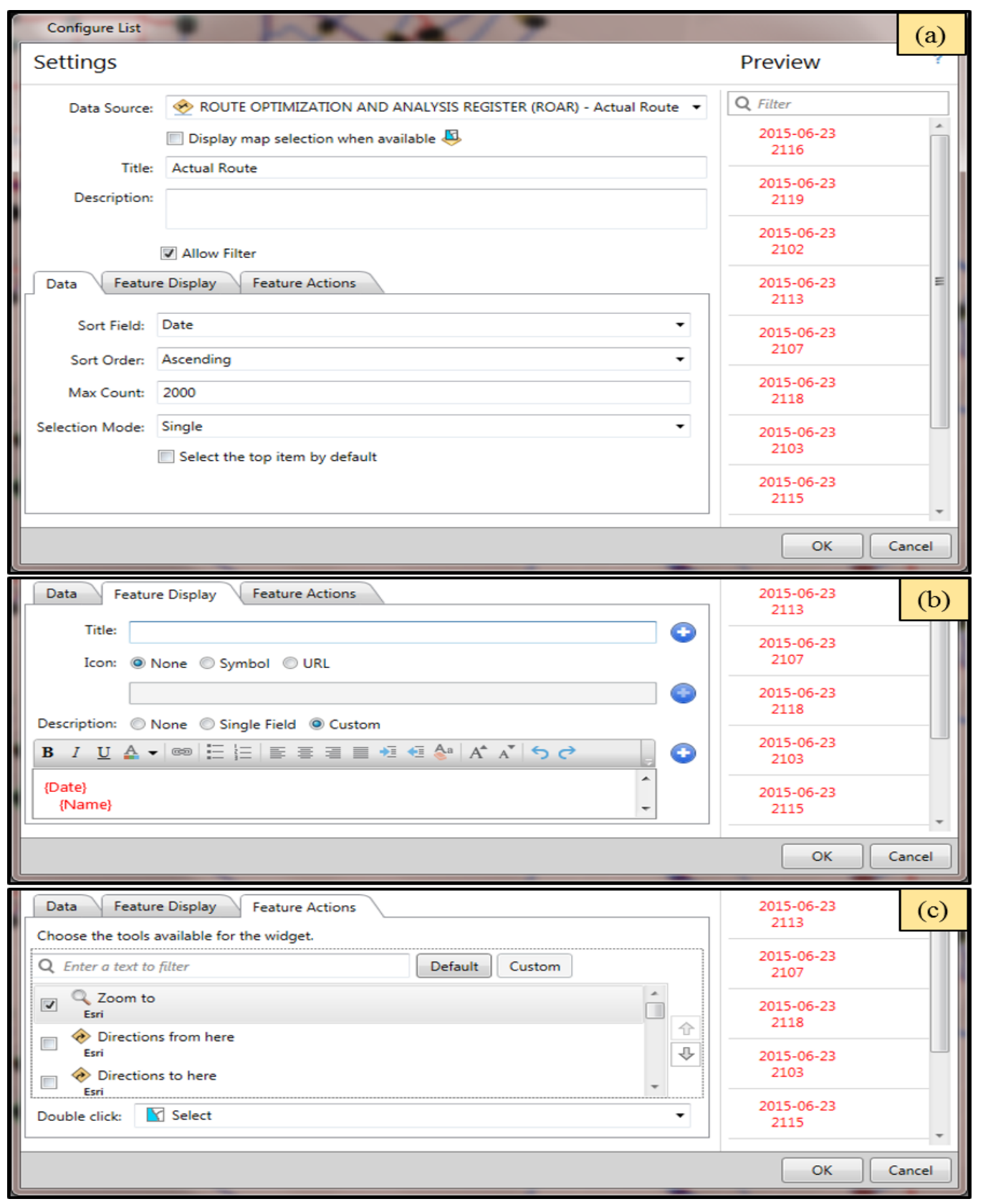

Figure 5-7: Configuring the List Widget.

\section{Data (a), Feature Display (b), and Feature Action (c)}

Two Indicator widgets were configured to display the KPIs related to time and distance displayed in Figure 5-8. Data source for these two indicator widgets were selected as the Actual Route Selection list. This allowed the user to see changes for different routes when clicking the features from the actual route list. Value field was set as Distance or Time and a required symbology was selected. In the appearance tab, trailing text was set as Miles or Minutes in order to display the unit on the dashboard. 


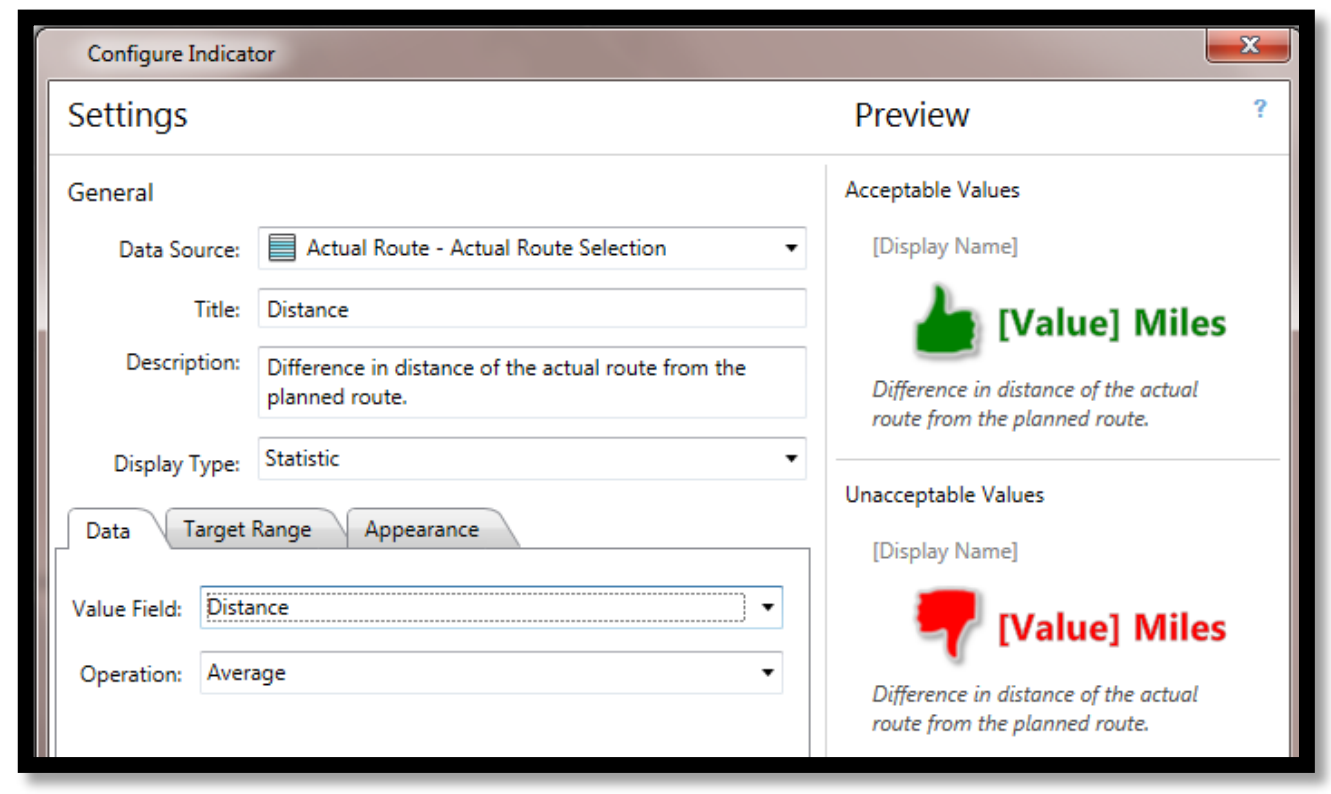

Figure 5-8: Configuring the Indicator Widget.

One Bar Chart widget was added to display the difference in the customer count from the two sets of routes, as illustrated in Figure 5-9. Like the indicator widgets, actual route list was selected as the bar chart data source. ActualStoresVisited and RecommendedStoresCount were the two fields selected in order to display the customer count difference on the dashboard. Color combinations were selected based on the client's requirements. Using the same process, one more Bar Chart widget was selected to display the time allocations of the recommended routes. Finally, all the widgets were arranged based on the final discussion with client. Figure 5-10 shows how the dashboard looks after arranging and placing the widgets. 


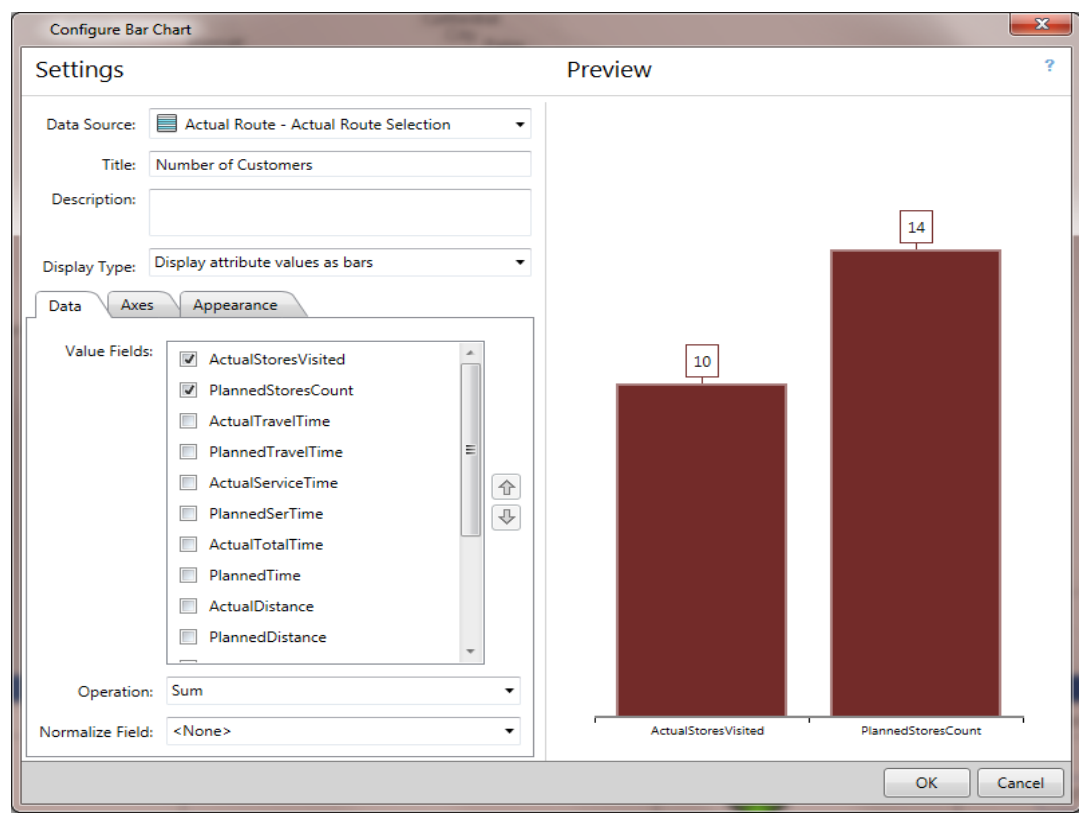

Figure 5-9: Configuring the Indicator Widget.

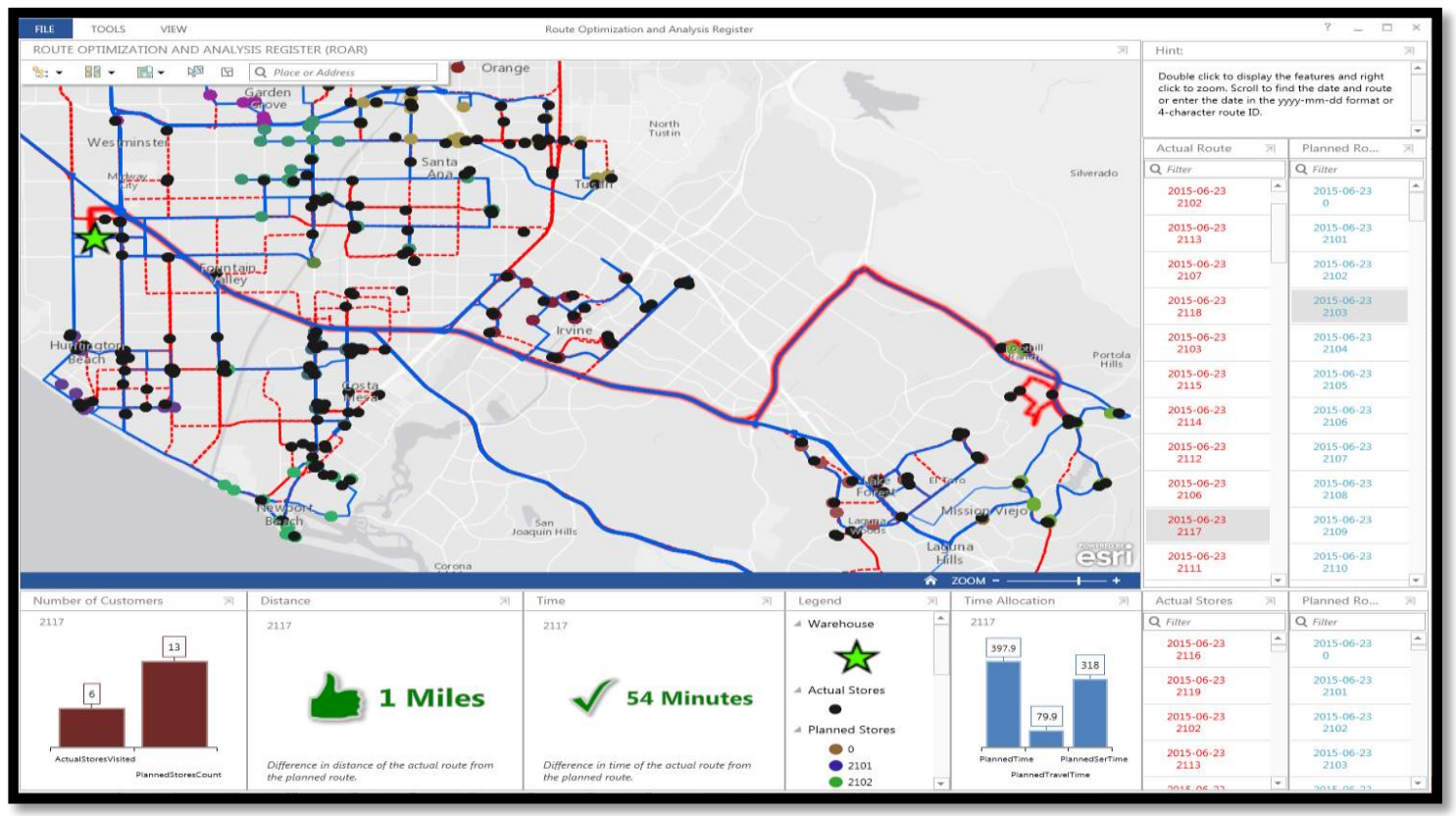

Figure 5-10: Configuring the Dashboard

\subsection{Summary}

This chapter discussed the modules, structure, and execution of the VRP solver for the ROAR in order to generate recommended routes, approximate actual routes, and the corresponding customers. Finally, it described the multiple configurations for a windows system, enterprise geodatabase, ArcGIS for Server, ArcGIS Online, and Operations Dashboard used by ROAR. 


\section{Chapter 6 - Use Case and Discussion}

This chapter presents outcome of the project. Section 6.1 discusses the results from recommended route script. Section 6.2 illustrates the findings from the actual route script. Section 6.3 summarizes the chapter.

\subsection{Use Case}

Route Optimization and Analysis Register (ROAR) would generate the output routes, recommended and actual, on a daily basis at 12:30 AM and 11:30 PM respectively. This section discusses the results generated by ROAR for a test case performed on May 14, 2015. To ensure that the scripts could run successfully, testing was conducted both manually and automatically.

\subsubsection{Recommended Route}

The existing tool that the client was using to generate the recommended routes took over 40 hours to generate the results, which included manual interventions. The ROAR script selected the data, formatted them, used ArcGIS Network Analyst extension, and generated the routes. The routes were created using constraints such as service time, time windows, truck capacity, delivery volume, max customer count, service week, and service day. The results from configuring and running the Network Analyst Vehicle Routing Problem (VRP) solver for existing routes were reviewed and verified. Two of the objective functions were to minimize the distance and to minimize the time. For a specified day, the script ran from the Windows' scheduled task at 12:30 AM. Figure 6-1 shows the progress message displayed on the console. The message confirmed the start of recommended routes generation.

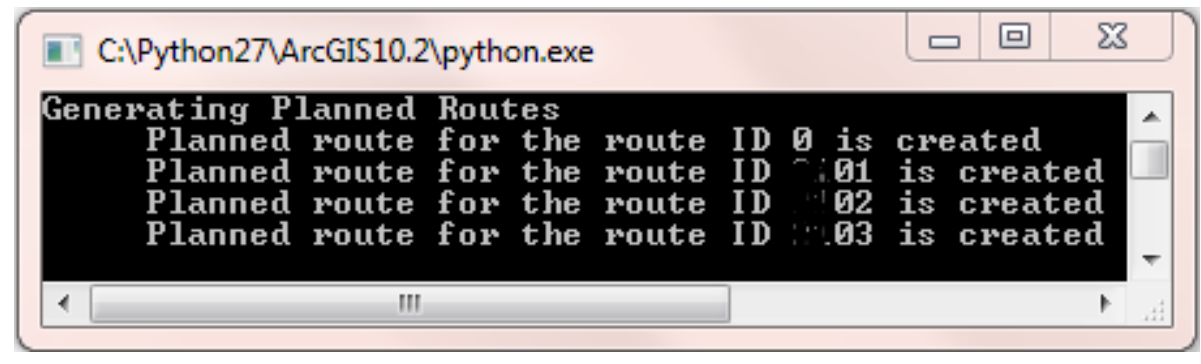

Figure 6-1: Display Message While Generating Recommended Routes

Customers to be serviced were automatically identified based upon the service day and the service week. For this test case, the day selected was Thursday in week three of a month. Figure 6-2 shows the selected customers and total customers. A subset of 296 out of 1,943 customers that were planned to be served on the day was selected. The selected customers were grouped based upon the 20 different pre-assigned route IDs. 


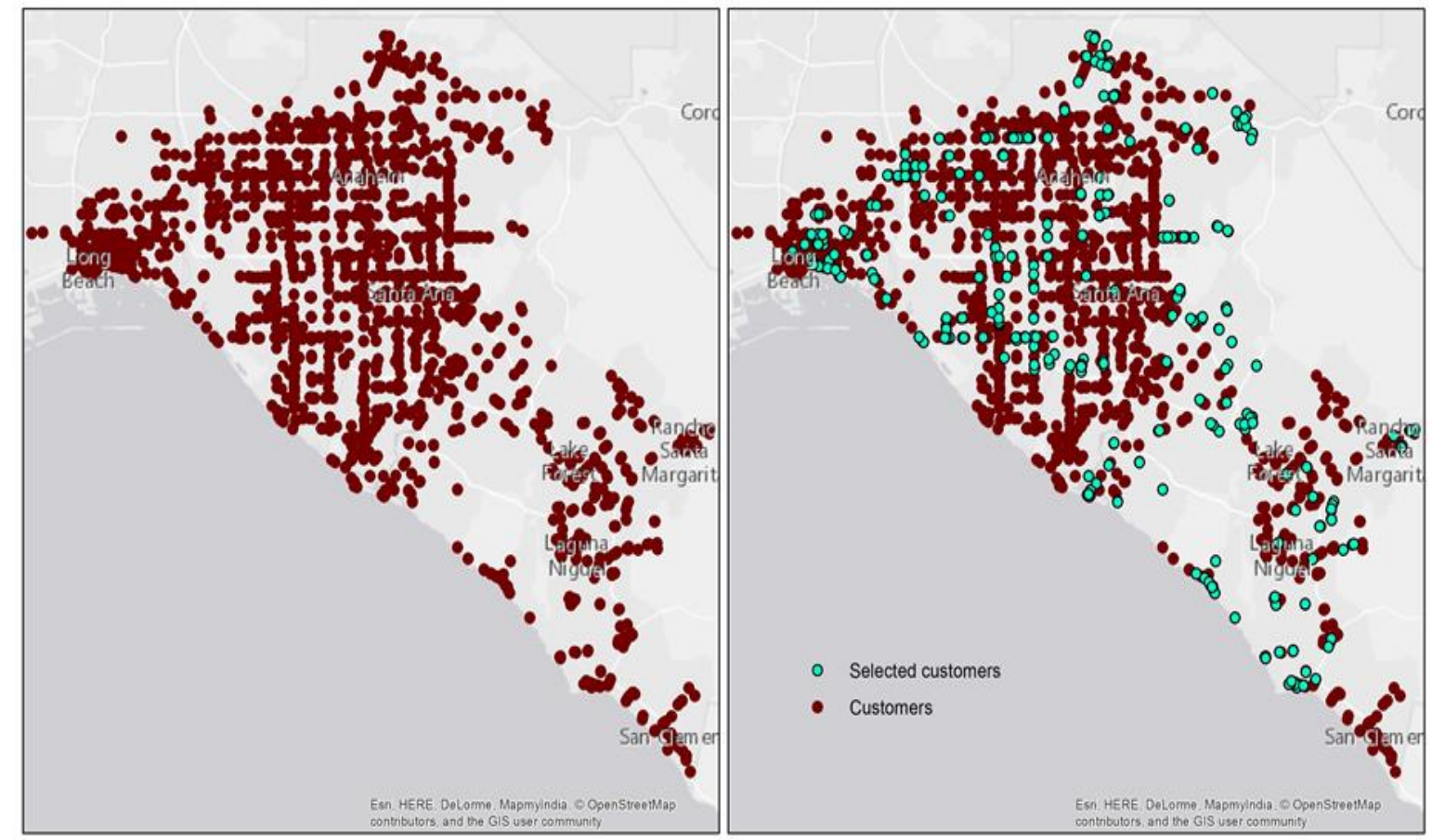

Figure 6-2: Total Customers and Those to be Serviced on Thursday in Week 3

The Vehicle Routing Problem (VRP) solver ran once for each group to generate the recommended routes. Figure 6-3 displays the generated recommended routes for all the trucks associated with 20 pre-defined route ID groups within the study area. Each route was associated with one corresponding customer group. 


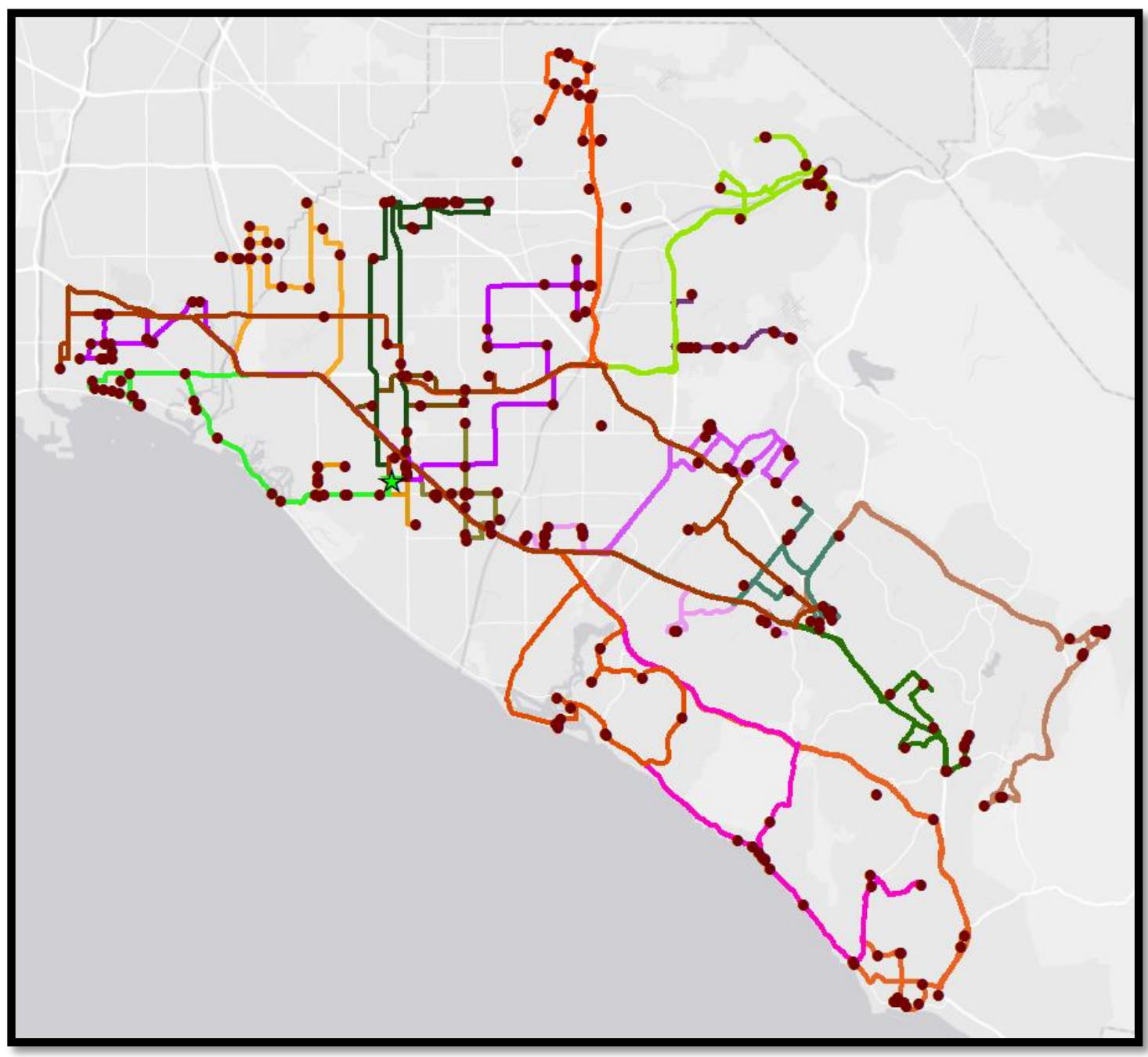

Figure 6-3: Recommended Routes for the Use Case

More detailed testing was conducted on individual routes. Figure 6-4 identifies one of the generated recommended routes, for the route ID 0011. Testing the individual routes provided details for service time, travel time, total time, distance travelled, and number of customers planned to be served. 


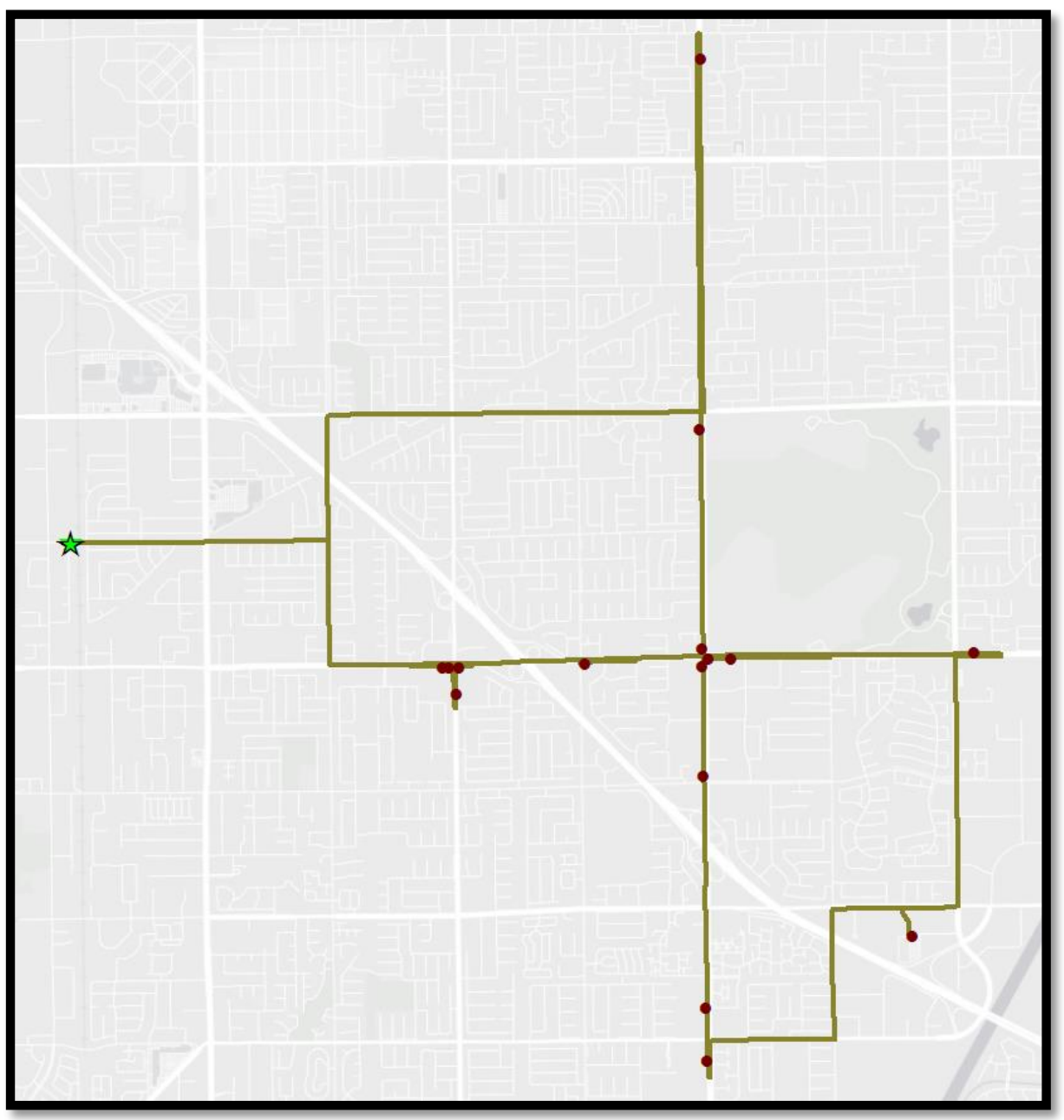

Figure 6-4: Recommended Route and Corresponding Customers for Route ID 0011

The recommended route script was run manually to generate routes for 20 groups of customers. Appendix B displays all the routes and the attributes attached to the routes and the recommended customer stops.

\subsubsection{Actual Route}

ROAR generated an approximation of the actual routes by connecting the extracted GPS points from Zonar, a third party fleet management system. These routes were previously visible only in the Zonar system, which required at least ten clicks to display the actual routes and download the GPS data. Like the recommended route, the actual route generation was automated using a Python script. Figure 6-5 shows the downloaded GPS data in CSV files for May 14, 2015. 


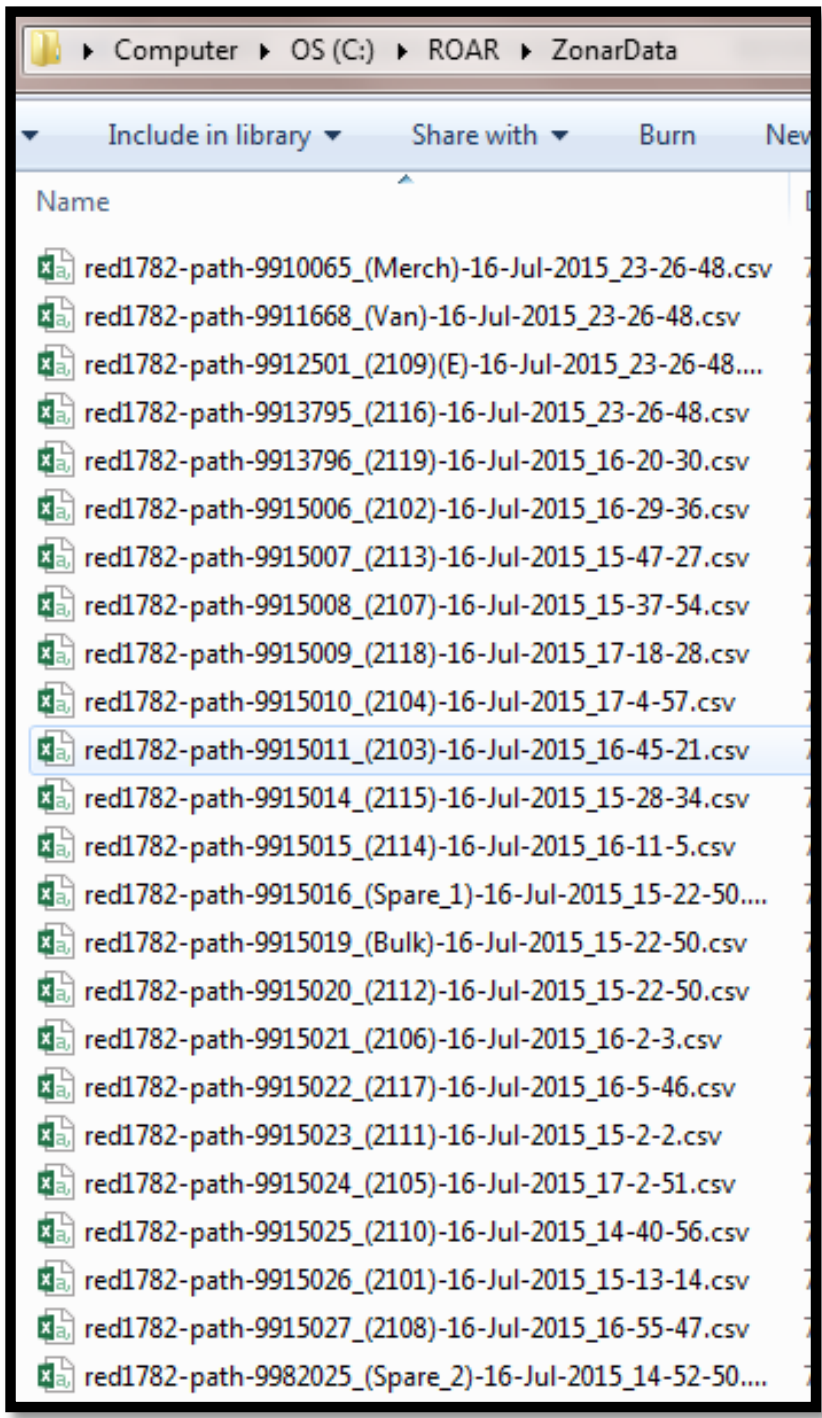

Figure 6-5: CSV Files in the Project Folder

Each file stored details such as asset ID, time for the log, speed, log reason, latitude, and longitude. Table 6 displays the contents that a downloaded CSV file stored. Contents from the CSV file were used to calculate the total time, distance travelled, and customer count for the actual route. 
Table 6. Contents of the CSV Files

\begin{tabular}{|l|l|l|l|l|l|l|l|}
\hline $\begin{array}{l}\text { Asset } \\
\text { ID }\end{array}$ & Date & $\begin{array}{l}\text { Time } \\
\text { (PDT) }\end{array}$ & $\begin{array}{l}\text { Speed } \\
\text { (MPH) }\end{array}$ & Heading & Log Reason & Lat & Lon \\
\hline 0011 & $5 / 14 / 2015$ & $7: 19: 06$ & 2.3 & North & $\begin{array}{l}\text { Power On, } \\
\text { Motion } \\
\text { Start }\end{array}$ & 33.7244709 & -117.9983784 \\
\hline 0011 & $5 / 14 / 2015$ & $7: 19: 34$ & 0 & $\begin{array}{l}\text { South } \\
\text { West }\end{array}$ & Power Off & 33.7242933 & -117.9986955 \\
\hline 0011 & $5 / 14 / 2015$ & $7: 29: 30$ & 5.1 & West & $\begin{array}{l}\text { Power On, } \\
\text { Start }\end{array}$ & 33.7242458 & -117.9989676 \\
\hline 0011 & $5 / 14 / 2015$ & $7: 31: 27$ & 0 & North & Power Off & 33.7243924 & -117.9990002 \\
\hline 0011 & $5 / 14 / 2015$ & $7: 40: 32$ & 0.3 & North & $\begin{array}{l}\text { Motion } \\
\text { Start }\end{array}$ & 33.7243933 & -117.998957 \\
\hline 0011 & $5 / 14 / 2015$ & $7: 43: 28$ & 13.7 & West & Standard & 33.7247548 & -117.9992361 \\
\hline 0011 & $5 / 14 / 2015$ & $7: 43: 46$ & 7.8 & West & Standard & 33.7247391 & -117.9999692 \\
\hline 0011 & $5 / 14 / 2015$ & $7: 43: 51$ & 0 & West & $\begin{array}{l}\text { Motion } \\
\text { Stop }\end{array}$ & 33.7247387 & -118.00008 \\
\hline 0011 & $5 / 14 / 2015$ & $7: 44: 08$ & 6.3 & $\begin{array}{l}\text { North } \\
\text { West }\end{array}$ & $\begin{array}{l}\text { Motion } \\
\text { Start }\end{array}$ & 33.7247619 & -118.0001167 \\
\hline 0011 & $5 / 14 / 2015$ & $7: 44: 26$ & 39.3 & North & Standard & 33.726736 & -118.0002082 \\
\hline 0011 & $5 / 14 / 2015$ & $7: 44: 44$ & 26.5 & North & Standard & 33.7293135 & -118.0002666 \\
\hline 0011 & $5 / 14 / 2015$ & $7: 44: 53$ & 0 & North & $\begin{array}{l}\text { Motion } \\
\text { Stop }\end{array}$ & 33.729693 & -118.0002827 \\
\hline
\end{tabular}

The actual routes were identified by selecting the downloaded files. The GPS points were connected sequentially and actual routes for all the trucks presented in Figure 6-6 were captured on May 14, 2015. The red lines are the actual routes that the truck took from the warehouse to deliver Red Bull products to assigned customers. 


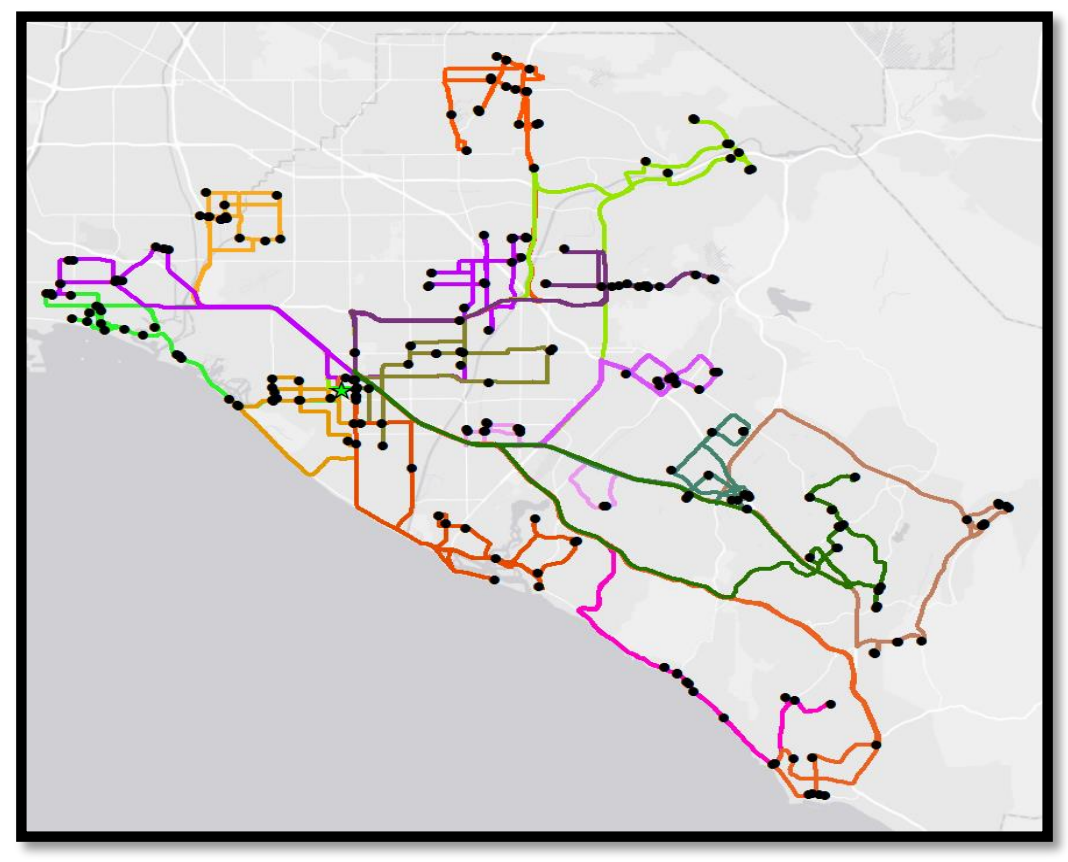

Figure 6-6: Actual Routes for the Use Case

More detailed testing was conducted on individual routes. Figure 6-7 identifies one of the actual routes, 0011 . Testing the individual routes provided details for service time, travel time, total time, distance travelled, and number of customers to be served.

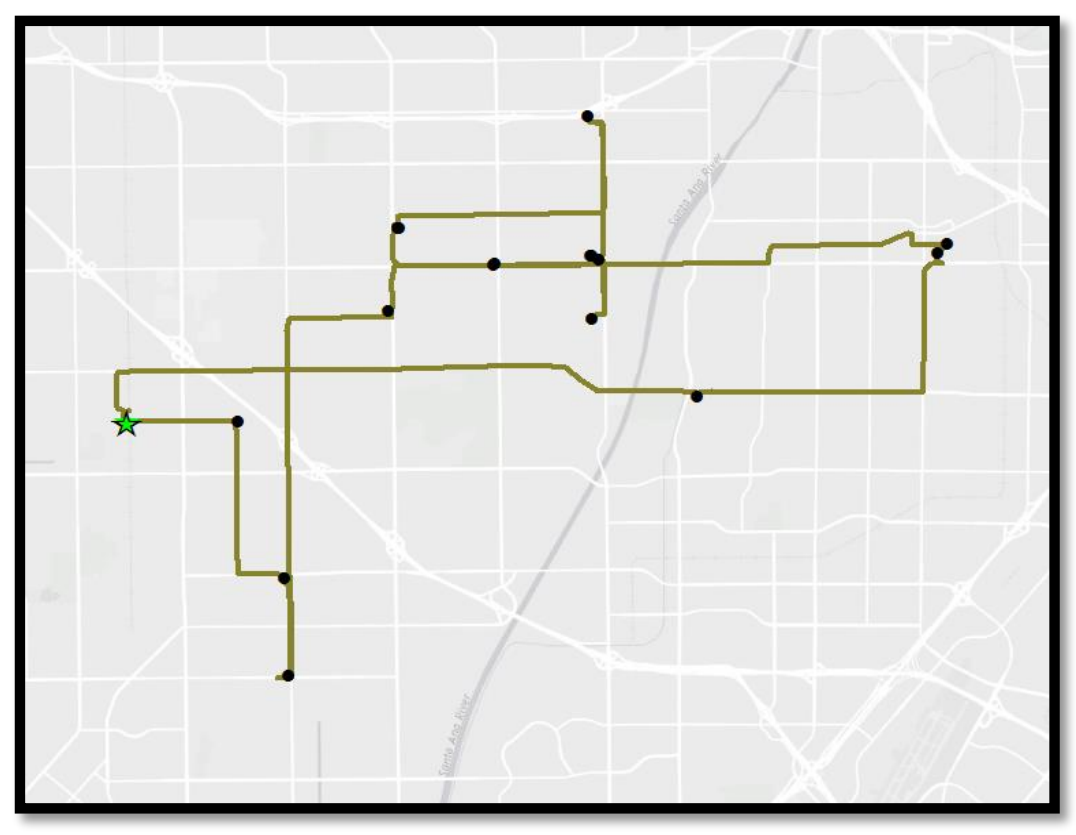

Figure 6-7: Actual Route and Corresponding Customers for Route ID 0011

The actual route script was run manually to identify the GPS points and the connecting routes. After adding the attributes such as time, distance, and customers 
served, Appendix $\mathrm{C}$ represents a sample output containing the actual routes details and the actual stops. The script copied the similar attributes from the recommended route feature class to the actual route feature class in order to perform the comparison between recommended and actual routes. Appendix D details the Key Performance Indicators (KPIs) results for recommended vs. actual routes for May 14, 2015.

\subsubsection{Operations Dashboard}

A map containing the recommended and the actual routes was displayed on the Operations Dashboard. Figure 6-8 displays the layout of the dashboard. The dashboard consisted of legend, recommended and actual routes, and KPI.

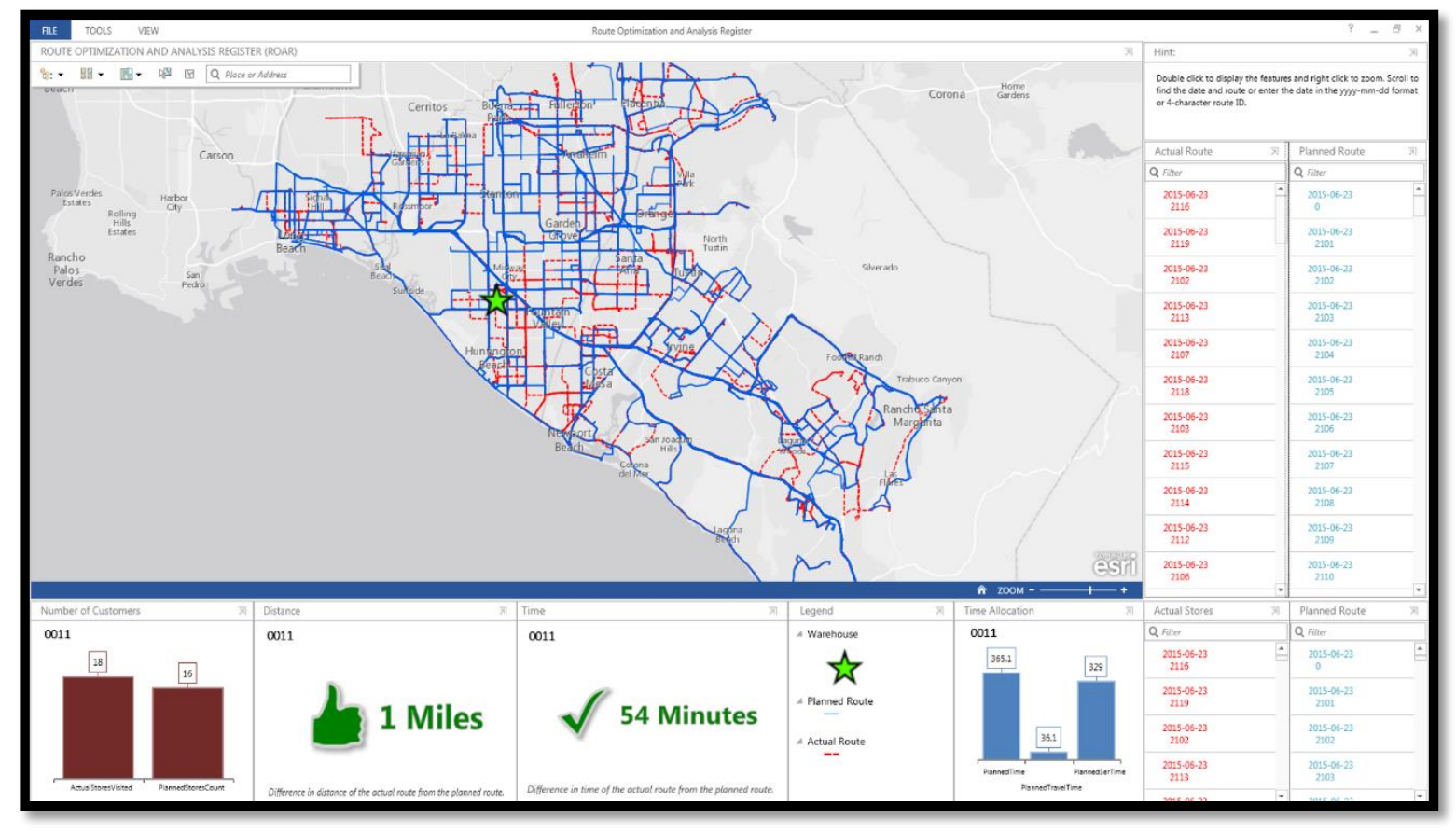

Figure 6-8: Operations Dashboard

The dashboard displayed the comparison results of the actual and recommended routes for May 14, 2015 for the route ID 0011. Results conclude that the truck travelled a mile less than what was recommended. In addition, the truck took 54 minutes less to complete the route compared to the recommended time.

\subsection{Discussion}

It was planned to use the "New Route" algorithm of ArcGIS Network Analyst extension to identify the actual routes, which would have allowed creating the actual routes along the streets to maintain the ground reality like curved turns. Upon verification from the Zonar dashboard, however, the Network Analyst created routes did not match. The GPS points collected by the device were not always accurate. Near a junction of the freeway and exit ramps, it was found on several occasions that the GPS points were closer to the 
ramp. Since the tool snapped to the nearest street, the Network Analyst route took the exit ramp when in reality it continued along the freeway. Figure 6-9 illustrates the issue.

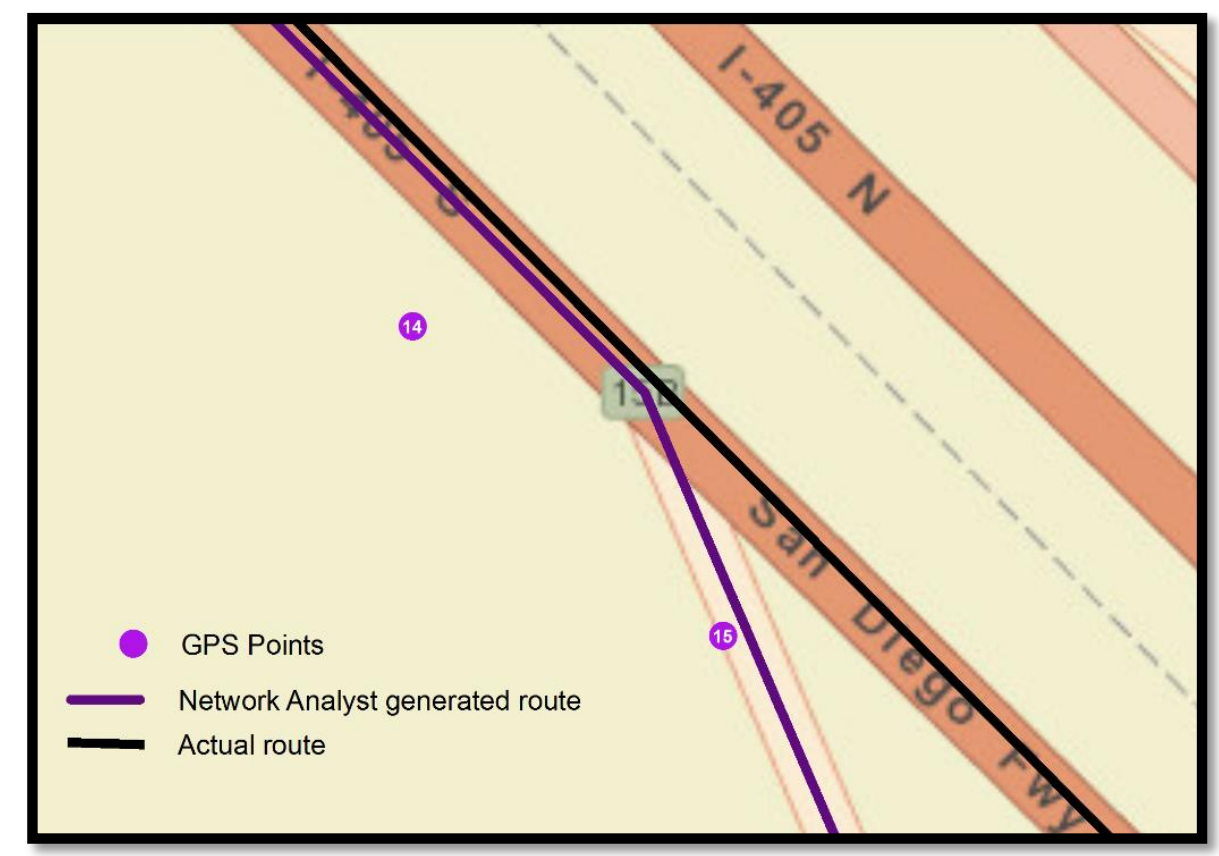

\section{Figure 6-9: GPS Points Identified Incorrectly}

There could be repeated routes in certain sections of the streets. For example, there were three points in a row supposedly on the freeway where the second point was near the ramp. Due to the issue discussed earlier, the generated route was created by taking the freeway exit to connect point one. If the third point was between the second point and the freeway entrance, then the truck had to take the entrance ramp that connected the other side of the freeway. This increased the travel time and distance on the actual route as a certain section of the street would be repeated unnecessarily.

To avoid these issues it was decided to connect the GPS points by the geoprocessing tool "Point to Line." This allowed the generated routes to match the Zonar routes although there was one limitation in this solution. Figure 6-10 explains the issue. 


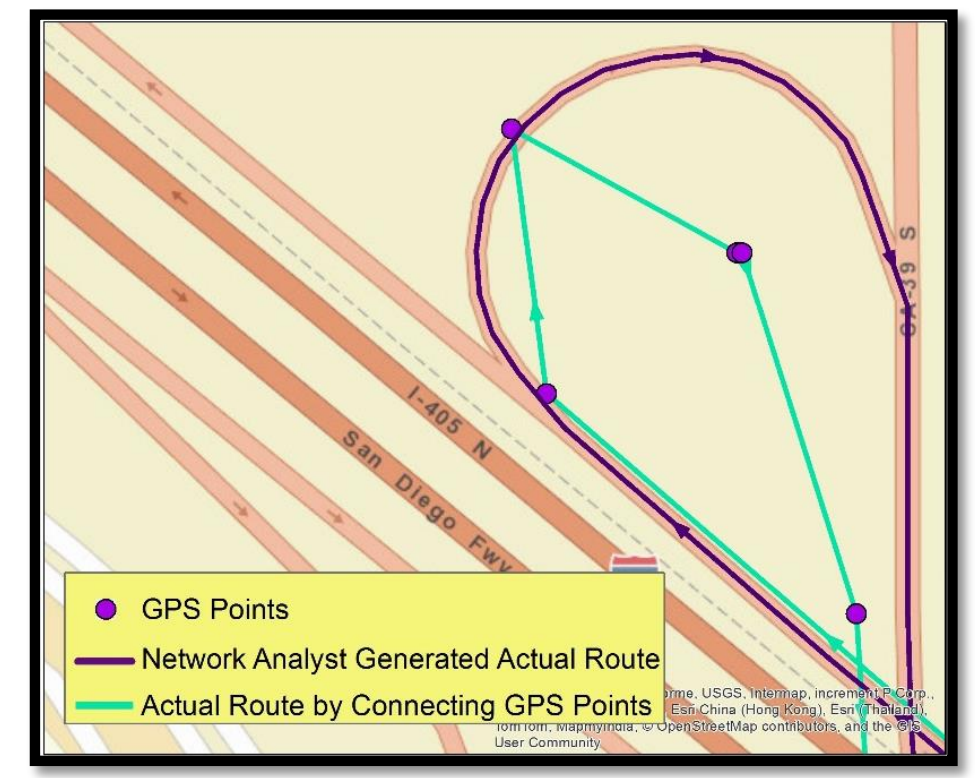

Figure 6-10: Route Created by Connecting the GPS Points

At different turnings, the route looked like sharp junctions connecting the different lines when it was supposed to have a curve along the street. Since the routes were the actual path that the truck took, it was more significant to identify the associated attributes like time and distance.

\subsection{Summary}

Compared to the previous system, ROAR generated optimized recommended routes within ten minutes compared to the previous tool that generated the optimized recommended routes in more than 40 hours. The GPS points of the delivery trucks were automatically downloaded from third party XML web services and the actual routes were identified from the extracted GPS points. Both set of routes were generated daily. The KPIs were calculated from the differences between actual and recommended routes. The results were displayed on Operations Dashboard and shared among the sales and marketing teams. Cumulatively, ROAR took less than an hour to complete the process. 


\section{Chapter 7 - Conclusions and Future Work}

This project allowed the Red Bull Corporation to evaluate how GIS technology could be used to generate efficient routes for its distribution trucks. The organization has a GIS department that can assess and implement the project findings in their testing environment. There were 24 delivery trucks distributing Red Bull product in the study area. This included both active and inactive trucks. Route Optimization and Analysis Register (ROAR) used Esri's Network Analyst extension and its Vehicle Routing Problem (VRP) solver to generate the recommended routes for the distribution trucks. ROAR also extracted the data from Zonar Fleet Management web services and produced the actual routes. After creating the two sets of routes, the tool compared them and displayed the result on a dashboard.

The goal of this project was to reduce the recommended route generation time. This goal was accomplished by ROAR with a Python script that used VRP solver. Prior to the creation of ROAR, the client took approximately 40 hours to generate the recommended routes. The tool generates the recommended routes in less than 10 minutes. Recommended routes are generated only for the active trucks.

The second goal for the project was to create an automated system that would allow users to analyze the actual routes compared to the recommended routes. To achieve this goal, the first script generating the recommended routes was used to automate the process. A second Python script was developed that extracts the GPS points of the delivery trucks from the Zonar Fleet Management system. The actual routes were generated from the extracted GPS points. Finally, the script compared the routes to calculate the Key Performance Indicators (KPIs). The difference between the recommended routes and the actual routes were displayed on a dashboard.

This project provided a starting point for future work. The results of this project show the Operations Dashboard is not ideal for displaying recommended versus actual routes. One option for future work is that the corporation could develop its own dashboard application that would allow the result to be displayed in way that is more customizable.

Access to the actual customers visited was not available in this project. Future work could be adding new parameter in the KPIs and calculating the efficiency of each route. The change would provide better results. 


\section{Works Cited}

Burns, L. D., Hall, R. W., Blumenfeld, D. E., \& Daganzo, C. F. (1985, May - June). Distribution Strategies that Minimize Transportation and Inventory Costs. Operations Research, 33(3), 469-490. Retrieved from http://www.jstor.org/stable/170552

Carić, T., \& Gold, H. (2008). Vehicle Routing Problem. Vienna, Austria: I-Tech. Retrieved from http://bib.irb.hr/datoteka/433524.Vehnicle_Routing_Problem.pdf

Chang, Y., \& Chen, L. (2007). Solve the vehicle routing problem with time windows via a genetic algorithm. Discrete and Continuous Dynamical Systems Supply, 240249.

Costley, M., Ruiz, R. F., Shi, H., Wong, M., \& Huynh, K. (n.d.). Red Bull's Company Synopsis. Retrieved November 29, 2014, from http://www.sfu.ca/ sheppard/478/syn/1131/Group_4.pdf

Dantzig, G. B., \& Ramser, J. H. (1959, October). The truck dispatching problem. Management Science, 6(1), 80-91. Retrieved from http://www.jstor.org/stable/2627477

Davis, A. D. (2013). A Route Generation Toolset for Produce Distribution. Master's Thesis, University of Redlands.

Dorigo, M., \& Gambardella, L. M. (1997). Ant colonies for the travelling salesman problem. BioSystems, 43(2), 73-81.

Esri. (2015). Operations Dashboard for ArcGIS. Retrieved July 02, 2015, from http://doc.arcgis.com/en/operations-dashboard/

Esri. (n.d.). ArcGIS Network Analyst. Retrieved June 25, 2015, from http://www.esri.com/software/arcgis/extensions/networkanalyst

Karadimas, N. V., Kolokathi, M., Defteraiou, G., \& Loumas, V. (2007). Municipal Waste Collection of Large Items Optimized with ArcGIS Network Analyst. 21st European Conference on Modelling and Simulation. Prague. Retrieved from http://www.researchgate.net/publication/229007213_Municipal_Waste_Collectio n_of_Large_Items_Optimized_With_ArcGIS_Network_Analyst

Kumar, S., \& Panneerselvam, R. (2012, May). A Survey on the Vehicle Routing Problem and Its Variants. Intelligent Information Management, 4(3), 66-74. Retrieved from http://www.scirp.org/journal/PaperDownload.aspx?DOI=10.4236/iim.2012.43010 
Montgomery, D. B. (1975, August). New Product Distribution: An Analysis of Supermarket Buyer Decisions. Journal of Marketing Research, 12(3), 255-264. Retrieved from http://www.jstor.org/stable/3151223

Munnik, R. (2013). Measuring the future of cloud-based GIS analysis. SASGI Proceedings. Retrieved from http://www.ee.co.za/wpcontent/uploads/2014/05/Rudolf-de-Munnik.pdf

O'Connor, D. L. (2013). Solid Waste Collection Vehicle Route Optimization for the City of Redlands, California. Master's Thesis, University of Redlands.

Red Bull. (n.d.). The Company Behind The Can. Retrieved July 30, 2015, from http://energydrink-us.redbull.com/company

van Elzakker, M. A., Maia, L. K., Zondervan, E., Raikar, N. B., \& Hoogland, H. (2014). Considering both Environmental Impact and Economic Costs in the Optimization of the Tactical Planning for the Fast Moving Consumer Goods Industry. Carnegie Institute of Technology, Department of Chemical Engineering. Carnegie Mellon University Research Showcase @ CMU. Retrieved from http://repository.cmu.edu/cgi/viewcontent.cgi?article $=1265 \&$ context $=$ cheme 


\section{Appendix A. Input Data for Recommended Route}

Table A-1. Customer Location Table

\begin{tabular}{|r|l|l|l|l|l|l|l|l|l|l|}
\hline $\begin{array}{l}\text { Location } \\
\text { ID }\end{array}$ & $\begin{array}{l}\text { Location } \\
\text { Name }\end{array}$ & $\begin{array}{l}\text { Location } \\
\text { Line 1 }\end{array}$ & City & $\begin{array}{l}\text { Zip } \\
\text { Code }\end{array}$ & Lat & Long & AT & STT & TWT & $\begin{array}{l}\text { Route } \\
\#\end{array}$ \\
\hline 1 & Cust1 & Address1 & City1 & z1 & & & G & T13 & G & 0018 \\
\hline 2 & Cust2 & Address2 & City2 & z2 & & & A & T1 & A & 0009 \\
\hline 16 & Cust16 & Address16 & City16 & z16 & & & E & T10 & E & 0000 \\
\hline 21 & Cust21 & Address21 & City21 & z21 & & & H & T6 & H & 0006 \\
\hline 24 & Cust24 & Address24 & City24 & z24 & & & D & T9 & D & 0006 \\
\hline 38 & Cust38 & Address38 & City38 & z38 & & & C & T4 & C & 0010 \\
\hline 618 & Cust618 & Address618 & City618 & z618 & & & B & T3 & B & 0004 \\
\hline 1205 & Cust1205 & Address1205 & City1205 & z1205 & & & F & T12 & F & 0003 \\
\hline
\end{tabular}

Table A-2. Service Time Table

\begin{tabular}{|l|l|l|l|r|r|}
\hline $\begin{array}{l}\text { Route } \\
\text { Type }\end{array}$ & $\begin{array}{l}\text { Customer } \\
\text { Abbreviation }\end{array}$ & $\begin{array}{l}\text { Account } \\
\text { Type }\end{array}$ & ID & Fixed & Variable \\
\hline T1 & A & A & T1 & 12.0 & 1.20 \\
\hline T2 & A & A & T2 & 22.0 & 1.20 \\
\hline T15 & A & A & T15 & 22.0 & 1.20 \\
\hline T16 & F & F & T16 & 45.0 & 0.87 \\
\hline T3 & B & B & T3 & 12.0 & 1.20 \\
\hline T4 & C & C & T4 & 20.0 & 1.20 \\
\hline T5 & C & C & T5 & 22.0 & 1.20 \\
\hline T6 & H & H & T6 & 20.0 & 0.87 \\
\hline T7 & H & H & T7 & 25.0 & 0.87 \\
\hline T8 & H & H & T8 & 35.0 & 0.87 \\
\hline T9 & D & D & T9 & 10.0 & 1.20 \\
\hline T10 & E & E & T10 & 22.0 & 0.87 \\
\hline T11 & E & E & T11 & 45.0 & 0.87 \\
\hline T12 & F & F & T12 & 22.0 & 1.20 \\
\hline T13 & G & G & T13 & 10.0 & 1.20 \\
\hline T14 & I & I & T14 & 5.0 & 1.20 \\
\hline
\end{tabular}


Table A-3. Volume Matric Table

\begin{tabular}{|r|l|l|l|l|l|l|}
\hline $\begin{array}{l}\text { Location } \\
\text { ID }\end{array}$ & $\begin{array}{l}\text { Territory } \\
\text { ID }\end{array}$ & Size 1 & Size 2 & Size 3 & AW & $\begin{array}{l}\text { Day } \\
\text { String }\end{array}$ \\
\hline 1 & 0018 & 3.16667 & 6.333333 & 13.71167 & XX & F \\
\hline 2 & 0009 & 6.17391 & 12.34783 & 26.73304 & XXXX & R \\
\hline 16 & 0000 & 72.4231 & 144.8462 & 313.5919 & XXXX & MR \\
\hline 21 & 0006 & 7.03846 & 14.07692 & 30.47654 & XXXX & W \\
\hline 24 & 0006 & 1.58333 & 3.166667 & 6.855833 & XX & T \\
\hline 38 & 0010 & 1.91304 & 3.826087 & 8.283478 & XX & R \\
\hline 618 & 0004 & 0.91667 & 1.833333 & 3.969167 & XX & F \\
\hline 1205 & 0003 & 2.68 & 5.36 & 11.6044 & XX & T \\
\hline
\end{tabular}

Table A-4. Time Window Table

\begin{tabular}{|c|c|c|c|c|c|c|c|c|}
\hline Type & Description & open & Close & TW1_open & TW1_close & TW2_open & TW2_close & Comment \\
\hline$A$ & $A$ & $\begin{array}{l}\text { 05:00:00 } \\
\text { AM }\end{array}$ & $\begin{array}{l}\text { 18:00:00 } \\
\text { PM }\end{array}$ & $\begin{array}{l}\text { 05:00:00 } \\
\text { AM }\end{array}$ & $\begin{array}{l}\text { 18:00:00 } \\
\text { PM }\end{array}$ & & & \\
\hline Z & Z & $\begin{array}{l}\text { 00:01:00 } \\
\text { AM }\end{array}$ & $\begin{array}{l}\text { 23:59:00 } \\
\text { PM }\end{array}$ & $\begin{array}{l}\text { 00:01:00 } \\
\text { AM }\end{array}$ & $\begin{array}{l}\text { 23:59:00 } \\
\text { PM }\end{array}$ & & & \\
\hline B & B & $\begin{array}{l}\text { 08:00:00 } \\
\text { AM }\end{array}$ & $\begin{array}{l}\text { 17:00:00 } \\
\text { PM }\end{array}$ & $\begin{array}{l}\text { 08:00:00 } \\
\text { AM }\end{array}$ & $\begin{array}{l}\text { 17:00:00 } \\
\text { PM }\end{array}$ & & & \\
\hline C & $\mathrm{C}$ & $\begin{array}{l}\text { 08:00:00 } \\
\text { AM }\end{array}$ & $\begin{array}{l}\text { 17:00:00 } \\
\text { PM }\end{array}$ & $\begin{array}{l}\text { 08:00:00 } \\
\text { AM }\end{array}$ & $\begin{array}{l}\text { 17:00:00 } \\
\text { PM }\end{array}$ & & & \\
\hline D & D & $\begin{array}{l}\text { 10:00:00 } \\
\text { AM }\end{array}$ & $\begin{array}{l}\text { 17:00:00 } \\
\text { PM }\end{array}$ & $\begin{array}{l}\text { 10:00:00 } \\
\text { AM }\end{array}$ & $\begin{array}{l}\text { 17:00:00 } \\
\text { PM }\end{array}$ & & & \\
\hline$E$ & $\mathrm{E}$ & $\begin{array}{l}\text { 05:00:00 } \\
\text { AM }\end{array}$ & $\begin{array}{l}\text { 10:00:00 } \\
\text { AM }\end{array}$ & $\begin{array}{l}\text { 05:00:00 } \\
\text { AM }\end{array}$ & $\begin{array}{l}\text { 10:00:00 } \\
\text { AM }\end{array}$ & & & \\
\hline $\mathrm{F}$ & $\mathrm{F}$ & $\begin{array}{l}\text { 06:00:00 } \\
\text { AM }\end{array}$ & $\begin{array}{l}\text { 10:00:00 } \\
\text { AM }\end{array}$ & $\begin{array}{l}\text { 06:00:00 } \\
\text { AM }\end{array}$ & $\begin{array}{l}\text { 10:00:00 } \\
\text { AM }\end{array}$ & & & \\
\hline G & G & $\begin{array}{l}\text { 08:00:00 } \\
\text { AM }\end{array}$ & $\begin{array}{l}\text { 17:00:00 } \\
\text { PM }\end{array}$ & $\begin{array}{l}\text { 08:00:00 } \\
\text { AM }\end{array}$ & $\begin{array}{l}\text { 17:00:00 } \\
\text { PM }\end{array}$ & & & \\
\hline $\mathrm{H}$ & $\mathrm{H}$ & $\begin{array}{l}\text { 05:00:00 } \\
\text { AM }\end{array}$ & $\begin{array}{l}\text { 10:00:00 } \\
\text { AM }\end{array}$ & $\begin{array}{l}\text { 05:00:00 } \\
\text { AM }\end{array}$ & $\begin{array}{l}\text { 10:00:00 } \\
\text { AM }\end{array}$ & & & \\
\hline I & 1 & $\begin{array}{l}\text { 08:00:00 } \\
\text { AM }\end{array}$ & $\begin{array}{l}\text { 17:00:00 } \\
\text { PM }\end{array}$ & $\begin{array}{l}\text { 08:00:00 } \\
\text { AM }\end{array}$ & $\begin{array}{l}\text { 17:00:00 } \\
\text { PM }\end{array}$ & & & \\
\hline
\end{tabular}




\section{Appendix B. Recommended Route Generated on May 14, 2015}

Table B-1. Recommended Stops Feature Class

\begin{tabular}{|l|l|r|}
\hline Shape * & Date & Name \\
\hline Multipoint & $5 / 14 / 2015$ & 0000 \\
\hline Multipoint & $5 / 14 / 2015$ & 0001 \\
\hline Multipoint & $5 / 14 / 2015$ & 0002 \\
\hline Multipoint & $5 / 14 / 2015$ & 0003 \\
\hline Multipoint & $5 / 14 / 2015$ & 0005 \\
\hline Multipoint & $5 / 14 / 2015$ & 0006 \\
\hline Multipoint & $5 / 14 / 2015$ & 0007 \\
\hline Multipoint & $5 / 14 / 2015$ & 0008 \\
\hline Multipoint & $5 / 14 / 2015$ & 0010 \\
\hline Multipoint & $5 / 14 / 2015$ & 0011 \\
\hline Multipoint & $5 / 14 / 2015$ & 0012 \\
\hline Multipoint & $5 / 14 / 2015$ & 0013 \\
\hline Multipoint & $5 / 14 / 2015$ & 0014 \\
\hline Multipoint & $5 / 14 / 2015$ & 0015 \\
\hline Multipoint & $5 / 14 / 2015$ & 0016 \\
\hline Multipoint & $5 / 14 / 2015$ & 0017 \\
\hline Multipoint & $5 / 14 / 2015$ & 0018 \\
\hline Multipoint & $5 / 14 / 2015$ & 0019 \\
\hline
\end{tabular}


Table B-2. Details of the Recommended Route

\begin{tabular}{|c|c|c|c|c|c|c|c|c|}
\hline Name & Date & $\begin{array}{l}\text { Order } \\
\text { Count }\end{array}$ & $\begin{array}{l}\text { Total } \\
\text { Time }\end{array}$ & $\begin{array}{l}\text { Total Order } \\
\text { Service } \\
\text { Time }\end{array}$ & $\begin{array}{l}\text { Total } \\
\text { Travel } \\
\text { Time } \\
\end{array}$ & $\begin{array}{l}\text { Total } \\
\text { Distance }\end{array}$ & Start Time & End Time \\
\hline 0000 & $5 / 14 / 2015$ & 9 & 299.97 & 188 & 111.97 & 75.90 & $\begin{array}{r}5 / 14 / 2015 \\
5: 24\end{array}$ & $\begin{array}{r}5 / 14 / 2015 \\
10: 24\end{array}$ \\
\hline 0001 & $5 / 14 / 2015$ & 17 & 391.59 & 335 & 56.59 & 30.32 & $\begin{array}{r}5 / 14 / 2015 \\
6: 39\end{array}$ & $\begin{array}{r}5 / 14 / 2015 \\
13: 10\end{array}$ \\
\hline 0002 & $5 / 14 / 2015$ & 16 & 393.98 & 337 & 56.98 & 35.41 & $\begin{array}{r}5 / 14 / 2015 \\
6: 00\end{array}$ & $\begin{array}{r}5 / 14 / 2015 \\
12: 34\end{array}$ \\
\hline 0003 & $5 / 14 / 2015$ & 18 & 451.10 & 386 & 65.10 & 38.50 & $\begin{array}{r}5 / 14 / 2015 \\
5: 42\end{array}$ & $\begin{array}{r}5 / 14 / 2015 \\
13: 13 \\
\end{array}$ \\
\hline 0004 & $5 / 14 / 2015$ & 16 & 385.22 & 327 & 58.22 & 29.63 & $\begin{array}{r}5 / 14 / 2015 \\
6: 34 \\
\end{array}$ & $\begin{array}{r}5 / 14 / 2015 \\
12: 59 \\
\end{array}$ \\
\hline 0005 & $5 / 14 / 2015$ & 15 & 381.15 & 304 & 77.15 & 51.35 & $\begin{array}{r}5 / 14 / 2015 \\
6: 17 \\
\end{array}$ & $\begin{array}{r}5 / 14 / 2015 \\
12: 38 \\
\end{array}$ \\
\hline 0006 & $5 / 14 / 2015$ & 13 & 447.06 & 355 & 92.06 & 63.85 & $\begin{array}{r}5 / 14 / 2015 \\
5: 05\end{array}$ & $\begin{array}{r}5 / 14 / 2015 \\
12: 32 \\
\end{array}$ \\
\hline 0007 & $5 / 14 / 2015$ & 15 & 376.75 & 317 & 59.75 & 42.08 & $\begin{array}{r}5 / 14 / 2015 \\
5: 40 \\
\end{array}$ & $\begin{array}{r}5 / 14 / 2015 \\
11: 57 \\
\end{array}$ \\
\hline 0008 & $5 / 14 / 2015$ & 16 & 425.89 & 363 & 62.89 & 36.96 & $\begin{array}{r}5 / 14 / 2015 \\
5: 55 \\
\end{array}$ & $\begin{array}{r}5 / 14 / 2015 \\
13: 01 \\
\end{array}$ \\
\hline 0009 & $5 / 14 / 2015$ & 17 & 342.86 & 309 & 33.86 & 16.19 & $\begin{array}{r}5 / 14 / 2015 \\
6: 28 \\
\end{array}$ & $\begin{array}{r}5 / 14 / 2015 \\
12: 11 \\
\end{array}$ \\
\hline 0010 & $5 / 14 / 2015$ & 15 & 359.93 & 329 & 30.93 & 17.15 & $\begin{array}{r}5 / 14 / 2015 \\
6: 29 \\
\end{array}$ & $\begin{array}{r}5 / 14 / 2015 \\
12: 29 \\
\end{array}$ \\
\hline 0011 & $5 / 14 / 2015$ & 16 & 365.07 & 329 & 36.07 & 37.21 & $\begin{array}{r}5 / 14 / 2015 \\
6: 20\end{array}$ & $\begin{array}{r}5 / 14 / 2015 \\
12: 25\end{array}$ \\
\hline 0012 & $5 / 14 / 2015$ & 17 & 477.66 & 410 & 67.66 & 45.94 & $\begin{array}{r}5 / 14 / 2015 \\
5: 15 \\
\end{array}$ & $\begin{array}{r}5 / 14 / 2015 \\
13: 13 \\
\end{array}$ \\
\hline 0013 & $5 / 14 / 2015$ & 15 & 379.11 & 311 & 68.11 & 49.25 & $\begin{array}{r}5 / 14 / 2015 \\
5: 05 \\
\end{array}$ & $\begin{array}{r}5 / 14 / 2015 \\
11: 24 \\
\end{array}$ \\
\hline 0014 & $5 / 14 / 2015$ & 15 & 378.50 & 322 & 56.50 & 39.27 & $\begin{array}{r}5 / 14 / 2015 \\
5: 55\end{array}$ & $\begin{array}{r}5 / 14 / 2015 \\
12: 14\end{array}$ \\
\hline 0015 & $5 / 14 / 2015$ & 14 & 353.06 & 281 & 72.06 & 39.20 & $\begin{array}{r}5 / 14 / 2015 \\
6: 16 \\
\end{array}$ & $\begin{array}{r}5 / 14 / 2015 \\
12: 09 \\
\end{array}$ \\
\hline 0016 & $5 / 14 / 2015$ & 12 & 374.34 & 305 & 69.34 & 56.25 & $\begin{array}{r}5 / 14 / 2015 \\
5: 21 \\
\end{array}$ & $\begin{array}{r}5 / 14 / 2015 \\
11: 35 \\
\end{array}$ \\
\hline 0017 & $5 / 14 / 2015$ & 13 & 456.25 & 364 & 92.25 & 81.65 & $\begin{array}{r}5 / 14 / 2015 \\
5: 25\end{array}$ & $\begin{array}{r}5 / 14 / 2015 \\
13: 02\end{array}$ \\
\hline 0018 & $5 / 14 / 2015$ & 13 & 389.00 & 298 & 91.00 & 65.98 & $\begin{array}{r}5 / 14 / 2015 \\
5: 31 \\
\end{array}$ & $\begin{array}{r}5 / 14 / 2015 \\
12: 00 \\
\end{array}$ \\
\hline 0019 & $5 / 14 / 2015$ & 14 & 408.59 & 325 & 83.59 & 72.00 & $\begin{array}{r}5 / 14 / 2015 \\
5: 39\end{array}$ & $\begin{array}{r}5 / 14 / 2015 \\
12: 27\end{array}$ \\
\hline
\end{tabular}




\section{Appendix C. Actual Route Generated on May 14, 2015}

Table C-1. Actual Stops Feature Class

\begin{tabular}{|l|l|r|}
\hline Shape * & Date & Name \\
\hline Multipoint & $5 / 14 / 2015$ & 0001 \\
\hline Multipoint & $5 / 14 / 2015$ & 0002 \\
\hline Multipoint & $5 / 14 / 2015$ & 0003 \\
\hline Multipoint & $5 / 14 / 2015$ & 0005 \\
\hline Multipoint & $5 / 14 / 2015$ & 0006 \\
\hline Multipoint & $5 / 14 / 2015$ & 0007 \\
\hline Multipoint & $5 / 14 / 2015$ & 0008 \\
\hline Multipoint & $5 / 14 / 2015$ & 0010 \\
\hline Multipoint & $5 / 14 / 2015$ & 0011 \\
\hline Multipoint & $5 / 14 / 2015$ & 0012 \\
\hline Multipoint & $5 / 14 / 2015$ & 0013 \\
\hline Multipoint & $5 / 14 / 2015$ & 0014 \\
\hline Multipoint & $5 / 14 / 2015$ & 0015 \\
\hline Multipoint & $5 / 14 / 2015$ & 0016 \\
\hline Multipoint & $5 / 14 / 2015$ & 0017 \\
\hline Multipoint & $5 / 14 / 2015$ & 0018 \\
\hline Multipoint & $5 / 14 / 2015$ & 0019 \\
\hline
\end{tabular}


Table C-2. Details of the Actual Route

\begin{tabular}{|r|l|r|r|r|r|r|}
\hline Name & Date & \multicolumn{1}{l|l|}{$\begin{array}{l}\text { Actual Stores } \\
\text { Visited }\end{array}$} & \multicolumn{1}{l|}{$\begin{array}{l}\text { Actual } \\
\text { Travel Time }\end{array}$} & $\begin{array}{l}\text { Actual } \\
\text { Service Time }\end{array}$ & $\begin{array}{l}\text { Actual } \\
\text { Total Time }\end{array}$ & $\begin{array}{l}\text { Actual } \\
\text { Distance }\end{array}$ \\
\hline 0001 & $5 / 14 / 2015$ & 10 & 163.90 & 347.35 & 511.25 & 47.20 \\
\hline 0002 & $5 / 14 / 2015$ & 20 & 237.43 & 346.70 & 584.13 & 39.50 \\
\hline 0003 & $5 / 14 / 2015$ & 14 & 264.28 & 352.93 & 617.22 & 35.70 \\
\hline 0005 & $5 / 14 / 2015$ & 13 & 106.18 & 367.87 & 474.05 & 55.30 \\
\hline 0006 & $5 / 14 / 2015$ & 11 & 188.52 & 369.38 & 557.90 & 61.60 \\
\hline 0007 & $5 / 14 / 2015$ & 10 & 146.67 & 379.92 & 526.58 & 44.30 \\
\hline 0008 & $5 / 14 / 2015$ & 17 & 145.70 & 336.20 & 481.90 & 42.00 \\
\hline 0010 & $5 / 14 / 2015$ & 19 & 100.33 & 375.22 & 475.55 & 26.00 \\
\hline 0011 & $5 / 14 / 2015$ & 17 & 29.07 & 282.00 & 311.07 & 36.10 \\
\hline 0012 & $5 / 14 / 2015$ & 11 & 123.52 & 381.95 & 505.47 & 39.40 \\
\hline 0013 & $5 / 14 / 2015$ & 10 & 111.40 & 408.68 & 520.08 & 37.30 \\
\hline 0014 & $5 / 14 / 2015$ & 13 & 142.73 & 403.62 & 546.35 & 36.10 \\
\hline 0015 & $5 / 14 / 2015$ & 13 & 142.80 & 365.20 & 508.00 & 41.60 \\
\hline 0016 & $5 / 14 / 2015$ & 11 & 147.67 & 342.70 & 490.37 & 60.90 \\
\hline 0017 & $5 / 14 / 2015$ & 6 & 139.53 & 204.67 & 344.20 & 63.10 \\
\hline 0018 & $5 / 14 / 2015$ & 7 & 97.95 & 370.27 & 468.22 & 54.10 \\
\hline 0019 & $5 / 14 / 2015$ & 10 & 137.10 & 379.52 & 516.62 & 81.10 \\
\hline
\end{tabular}




\section{Appendix D. Key Performance Indicators Table on May 14, 2015}

Table D-1. An Example of Key Performance Indicators

\begin{tabular}{|l|l|r|r|r|r|r|r|}
\hline Name & Date & $\begin{array}{l}\text { Actual } \\
\text { Store }\end{array}$ & \multicolumn{1}{l}{$\begin{array}{l}\text { Planned } \\
\text { Store }\end{array}$} & Travel Time & Service Time & Total Time & Distance \\
\hline 0001 & $5 / 14 / 2015$ & 16 & 17 & -115.646636 & -104.416667 & -220.063303 & -13.882875 \\
\hline 0002 & $5 / 14 / 2015$ & 18 & 16 & -111.770916 & -97.1 & -208.870916 & -0.192679 \\
\hline 0003 & $5 / 14 / 2015$ & 16 & 18 & -147.366178 & -40.6 & -187.966178 & -35.998832 \\
\hline 0004 & $5 / 14 / 2015$ & 4 & 16 & -122.332484 & 258.65 & 136.317516 & 27.234413 \\
\hline 0005 & $5 / 14 / 2015$ & 19 & 15 & -188.263219 & -131.866667 & -320.129886 & -22.851387 \\
\hline 0006 & $5 / 14 / 2015$ & 12 & 13 & -99.876229 & -87.15 & -187.026229 & -3.950705 \\
\hline 0007 & $5 / 14 / 2015$ & 15 & 15 & -108.696858 & -130.483333 & -239.180191 & -15.715677 \\
\hline 0008 & $5 / 14 / 2015$ & 19 & 16 & -111.911406 & -27.466667 & -139.378073 & -15.038741 \\
\hline 0009 & $5 / 14 / 2015$ & 19 & 15 & -113.46742 & -211.516667 & -324.984087 & -15.047495 \\
\hline 0010 & $5 / 14 / 2015$ & 18 & 16 & -96.650613 & -200.366667 & -297.01728 & -16.910083 \\
\hline 0011 & $5 / 14 / 2015$ & 10 & 17 & -108.821027 & -4.533333 & -113.354361 & -3.858575 \\
\hline 0012 & $5 / 14 / 2015$ & 13 & 15 & -150.520876 & -129.4 & -279.920876 & -4.745054 \\
\hline 0013 & $5 / 14 / 2015$ & 14 & 15 & -85.316926 & -122.766667 & -208.083592 & -6.034177 \\
\hline 0014 & $5 / 14 / 2015$ & 16 & 14 & -175.792613 & -104.216667 & -280.00928 & -19.001801 \\
\hline 0015 & $5 / 14 / 2015$ & 18 & 12 & -157.173842 & -139.066667 & -296.240508 & -16.94993 \\
\hline 0016 & $5 / 14 / 2015$ & 15 & 13 & -38.604756 & -65.366667 & -103.971423 & 6.247027 \\
\hline 0017 & $5 / 14 / 2015$ & 11 & 13 & -72.42985 & -35.633333 & -108.063184 & -1.023501 \\
\hline 0018 & $5 / 14 / 2015$ & 10 & 14 & -67.77671 & -80.45 & -148.22671 & -0.396182 \\
\hline
\end{tabular}




\section{Appendix E. Script for Creating Feature Classes in the Geodatabase}

\# system modules

import arcpy

\# Setting overwrite paramenter as true

arcpy.env.overwriteOutput $=$ True

\# define the variables

location = "C: $\backslash$ ROAR $\backslash$ ROARFleetManagement.sde"

actualRouteFC = "ActualRoute"

actualStore FC = "ActualStore"

plannedRouteFC = "PlannedRoute"

plannedStoreFC = "PlannedStore"

\# Define the spatial reference

spatialReference $=$

"PROJCS['WGS_1984_Web_Mercator_Auxiliary_Sphere',GEOGCS['GCS_WGS_1984', DATUM['D_WGS_1984',SPHEROID['WGS_1984',6378137.0,298.257223563]],PRIME M['Greenwich',0.0],UNIT['Degree',0.0174532925199433]],PROJECTION['Mercator_A uxiliary_Sphere'],PARAMETER['False_Easting',0.0],PARAMETER['False_Northing',0.0 ],PARAMETER['Central_Meridian',0.0],PARAMETER['Standard_Parallel_1',0.0],PAR AMETER['Auxiliary_Sphere_Type',0.0],UNIT['Meter',1.0]]"

\# create the empty feature classes

arcpy.CreateFeatureclass_management(location, actualRouteFC, "POLYLINE")

arcpy.DefineProjection_management(location + " $\backslash "$ " actualRouteFC,

spatialReference)

arcpy.RegisterAsVersioned_management(location + " $\backslash "+$ actualRouteFC)

arcpy.CreateFeatureclass_management(location, actualStoreFC, "MULTIPOINT")

arcpy.DefineProjection_management(location + " $\backslash "+$ actualStoreFC, spatialReference)

arcpy.RegisterAsVersioned_management(location + " $\backslash 1 "+$ actualStoreFC)

arcpy.CreateFeatureclass_management(location, plannedRouteFC, "POLYLINE")

arcpy.DefineProjection_management(location + " $\backslash "$ + plannedRouteFC,

spatialReference)

arcpy.RegisterAsVersioned_management(location + " $\backslash "$ + plannedRouteFC)

arcpy.CreateFeatureclass_management(location, plannedStoreFC, "MULTIPOINT")

arcpy.DefineProjection_management(location + " $\backslash "$ + plannedStoreFC,

spatialReference)

arcpy.RegisterAsVersioned_management(location + " $\backslash "$ + plannedStoreFC)

\# add fields for each feature class

arcpy.AddField_management(location + "ר" + actualRouteFC, "Name", "TEXT")

arcpy.AddField_management(location + "ר" + actualRouteFC, "Date", "TEXT")

arcpy.AddField_management(location + "ר\" + actualRouteFC, "ActualStoresVisited",

"SHORT") 
arcpy.AddField_management(location + "\\" + actualRouteFC, "PlannedStoresCount", "SHORT")

arcpy.AddField_management(location + "רI" + actualRouteFC, "ActualTravelTime", "DOUBLE")

arcpy.AddField_management(location + " $\backslash "$ + actualRouteFC, "PlannedTravelTime", "DOUBLE")

arcpy.AddField_management(location + "\" + actualRouteFC, "ActualServiceTime", "DOUBLE")

arcpy.AddField_management(location + "ר" + actualRouteFC, "PlannedSerTime",

"DOUBLE")

arcpy.AddField_management(location + "ר" + actualRouteFC, "ActualTotalTime", "DOUBLE")

arcpy.AddField_management(location + "१" + actualRouteFC, "PlannedTime",

"DOUBLE")

arcpy.AddField_management(location + "\" + actualRouteFC, "ActualDistance", "DOUBLE")

arcpy.AddField_management(location + "१" + actualRouteFC, "PlannedDistance", "DOUBLE")

arcpy.AddField_management(location + "ר" + actualRouteFC, "TravelTime", "DOUBLE")

arcpy.AddField_management(location + "ר" + actualRouteFC, "ServiceTime", "DOUBLE")

arcpy.AddField_management(location + "ר" + actualRouteFC, "TotalTime",

"DOUBLE")

arcpy.AddField_management(location + "ר" + actualRouteFC, "Distance", "DOUBLE")

arcpy.AddField_management(location + " $\backslash "+$ actualRouteFC, "Stores", "SHORT")

arcpy.AddField_management(location + "\" + actualRouteFC, "Efficiency", "FLOAT")

arcpy.AddField_management(location + " $\backslash "$ + plannedRouteFC, "Name", "TEXT")

arcpy.AddField_management(location + "ר" + plannedRouteFC, "Date", "TEXT")

arcpy.AddField_management(location + " $\backslash "$ + plannedRouteFC, "Description",

"TEXT")

arcpy.AddField_management(location + "\" + plannedRouteFC, "StartDepotName",

"TEXT")

arcpy.AddField_management(location + "\I" + plannedRouteFC, "EndDepotName",

"TEXT")

arcpy.AddField_management(location + " $\backslash "+$ plannedRouteFC,

"StartDepotServiceTime", "DOUBLE")

arcpy.AddField_management(location + " $\backslash "+$ plannedRouteFC,

"EndDepotServiceTime", "DOUBLE")

arcpy.AddField_management(location + "ר" + plannedRouteFC, "EarliestStartTime",

"DATE")

arcpy.AddField_management(location + "ר" + plannedRouteFC, "LatestStartTime",

"DATE")

arcpy.AddField_management(location + " $\backslash "$ + plannedRouteFC, "ArriveDepartDelay", "DOUBLE")

arcpy.AddField_management(location + "\" + plannedRouteFC, "Capacities", "TEXT") 
arcpy.AddField_management(location + "ר" + plannedRouteFC, "FixedCost", "DOUBLE")

arcpy.AddField_management(location + " $\backslash "$ + plannedRouteFC, "CostPerUnitTime", "DOUBLE")

arcpy.AddField_management(location + " $\backslash "$ + plannedRouteFC,

"CostPerUnitDistance", "DOUBLE")

arcpy.AddField_management(location + " $\backslash "$ + plannedRouteFC, "OvertimeStartTime", "DOUBLE")

arcpy.AddField_management(location + "ר" + plannedRouteFC,

"CostPerUnitOvertime", "DOUBLE")

arcpy.AddField_management(location + "ר" + plannedRouteFC, "MaxOrderCount", "LONG")

arcpy.AddField_management(location + "ר" + plannedRouteFC, "MaxTotalTime", "DOUBLE")

arcpy.AddField_management(location + " $\backslash "$ + plannedRouteFC,

"MaxTotalTravelTime", "DOUBLE")

arcpy.AddField_management(location + "ר" + plannedRouteFC, "MaxTotalDistance", "DOUBLE")

arcpy.AddField_management(location + "ר" + plannedRouteFC, "SpecialtyNames", "TEXT")

arcpy.AddField_management(location + "ר" + plannedRouteFC, "AssignmentRule ", "LONG")

arcpy.AddField_management(location + " $\backslash "$ + plannedRouteFC, "ViolatedConstraints", "LONG")

arcpy.AddField_management(location + "ר" + plannedRouteFC, "OrderCount", "LONG")

arcpy.AddField_management(location + " $\backslash "$ + plannedRouteFC, "TotalCost", "DOUBLE")

arcpy.AddField_management(location + " $\backslash "$ + plannedRouteFC, "RegularTimeCost", "DOUBLE")

arcpy.AddField_management(location + " $\backslash "$ + plannedRouteFC, "OvertimeCost", "DOUBLE")

arcpy.AddField_management(location + " $\backslash "$ + plannedRouteFC, "DistanceCost", "DOUBLE")

arcpy.AddField_management(location + "ר" + plannedRouteFC, "TotalTime",

"DOUBLE")

arcpy.AddField_management(location + " $\backslash "$ + plannedRouteFC,

"TotalOrderServiceTime", "DOUBLE")

arcpy.AddField_management(location + " $\backslash "$ + plannedRouteFC,

"TotalBreakServiceTime", "DOUBLE")

arcpy.AddField_management(location + "ר" + plannedRouteFC, "TotalTravelTime", "DOUBLE")

arcpy.AddField_management(location + " $\ "$ + plannedRouteFC, "TotalDistance", "DOUBLE")

arcpy.AddField_management(location + "ר" + plannedRouteFC, "StartTime", "DATE")

arcpy.AddField_management(location + "ר" + plannedRouteFC, "EndTime", "DATE") 
arcpy.AddField_management(location + " $\backslash "+$ plannedRouteFC, "TotalWaitTime", "DOUBLE")

arcpy.AddField_management(location + "ר" + plannedRouteFC, "TotalViolationTime", "DOUBLE")

arcpy.AddField_management(location + " $\backslash 1 "$ + plannedRouteFC, "RenewalCount", "LONG")

arcpy.AddField_management(location + " $\backslash "+$ plannedRouteFC,

"TotalRenewalServiceTime", "DOUBLE")

arcpy.AddField_management(location + " $\backslash "$ + plannedStoreFC, "Date", "TEXT")

arcpy.AddField_management(location + " $\backslash 1$ + plannedStoreFC, "Name", "TEXT")

arcpy.AddField_management(location + " $\backslash "+$ actualStoreFC, "Date", "TEXT")

arcpy.AddField_management(location + " $\backslash "+$ actualStoreFC, "Name", "TEXT") 


\title{
Appendix F. Script for Creating Planned Routes
}

\author{
\#system modules \\ import urllib2 \\ import xml.etree.cElementTree as etree \\ import os \\ import datetime, time \\ import arcpy \\ from datetime import date \\ import calendar \\ print (datetime.datetime.now()) \\ print ("Generating Planned Routes")
}

\# Capture the week number. Expected output is 1,2,3 and so on.

day_of_month = datetime.datetime.now ().day

week_number $=($ day_of_month -1$) / / 7+1$

\# No routes generated if week number greater than 4.

if week_number $>4$ :

print "Truck not supposed to run"

else:

\# Capture the day of the week. Expected output is Monday, Tuesday, and so on.

week_number $=$ str(week_number)

my_date $=$ date.today ()

Day = calendar.day_name[my_date.weekday()]

\# If the day is Saturday or Sunday no routes will be created.

if Day == 'Saturday' or Day == 'Sunday':

print "Enjoy your weekend!"

else:

\# Setting up the planned route geodatabase workspace for the input tables and the input feature classes.

arcpy.env.workspace $=$ "C:IVROARIIROARFleetManagement.sde"

arcpy.env.overwriteOutput $=$ True

\# Calling feature classes and tables.

Stores = "OC_Store" \#store locations from client

Base_Location_Extension = "LocationBaseline" \#Base location extension table

TimeWindow = "timeWindow"

\#Time window table

ServiceTime $=$ "serviceTime" \#Service time table

\# Local variables

Copied_Stores = "in_memoryllCopied_Stores"

Stores_with_BLE $=$ Copied_Stores

Stores_with_ST $=$ Stores_with_BLE

Stores_with_TWT $=$ Stores_with_ST

Stores_SeriveDay $=$ Stores_with_TWT

Stores_ServiceWeek = Stores_SeriveDay

Stores_With_SD = Stores_ServiceWeek 
Stores_With_SW $=$ Stores_With_SD

Stores_Volume $=$ Stores_With_SW

Stores_With_Vol = Stores_Volume

Stores_Check $=$ Stores_With_Vol

Stores_With_Check $=$ Stores_Check

Stores_Unique_ID = "in_memoryl|Stores_Unique_ID"

Final_Stores = "in_memoryllClean_Stores"

Day_Order = "in_memoryl|Day_Order"

BL_dbase = "in_memoryl|BLext"

ST_dbase = "in_memoryllST_dbase"

TWT_dbase = "in_memoryllTWT_dbase"

freqTable = "in_memoryl|routeIDs"

freqField $=$ "Route

plannedRouteFC = "C:I|ROAR\|ROARFleetManagement.sdel|PlannedRoute"

plannedStoreFC = "C:IVROARIIROARFleetManagement.sdel\PlannedStore"

\# Process: Copy Stores

arcpy.CopyFeatures_management(Stores, Copied_Stores, "", "0", "0", "0")

\# Process: BL Excel To dBase

arcpy.CopyRows_management(Base_Location_Extension, BL_dbase, "')

\# Process: Join BL

arcpy.JoinField_management(Copied_Stores, "Location_ID", BL_dbase,

"Location_ID", "Size_1;Size_2;Size_3;AW;Day_String")

\# Process: serviceTime Excel To dBase

arcpy.CopyRows_management(ServiceTime, ST_dbase, "')

\# Process: Join STT

arcpy.JoinField_management(Stores_with_BLE, "STT", ST_dbase, "ID",

"Customer_Abbreviation;Account_Type;ID;Fixed;Variable")

\# Process: timeWindow Excel To dBase

arcpy.CopyRows_management(TimeWindow, TWT_dbase, "")

\# Process: Join TWT

arcpy.JoinField_management(Stores_with_ST, "TWT", TWT_dbase, "type",

"type;open;close;TW1_open;TW1_close;TW2_open;TW2_close")

\# Process: Add Field ServiceDay

arcpy.AddField_management(Stores_with_TWT, "ServiceDay", "TEXT", "', "',

"6", "", "NULLABLE", "NON_REQUIRED", "")

\# Process: Add Field ServiceWK

arcpy.AddField_management(Stores_SeriveDay, "ServiceWK", "TEXT", "", "', "4",

"", "NULLABLE", "NON_REQUIRED", "")

\# Process: Calculate ServiceDay

arcpy.CalculateField_management(Stores_ServiceWeek, "ServiceDay",

"ServiceDay(!Day_String!)", "PYTHON_9.3", "def ServiceDay( val ):Inn if val ==

'M': In return '000001' In elif val == 'T': In return '000010'।n elif val ==

'W': \n return '000100' \n elif val == 'R': \n return '001000' $\backslash n$ elif val ==

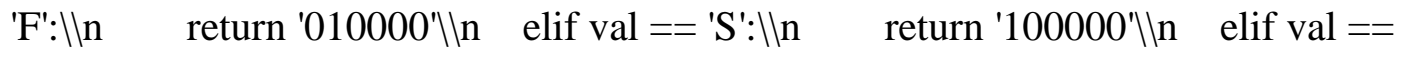

'MT':In return '000011'In elif val == 'MW': In return '000101' In elif val ==

'MR': \n return '001001' In elif val== 'MF': In return '010001' Inn elif val == 
'MS': In

'TR': Iln

'TS': IIn

'WF':Iln

'RF':Iln

'FS': \n return '100001' In elif val == 'TW': Iln return '001010' $\backslash n$ elif val == 'TF': $\backslash \mathrm{n}$ return '100010' $\backslash \mathrm{n}$ elif val $==$ 'WR': $\backslash \mathrm{n}$ return '010100' In elif val == 'WS': IIn return '011000' \n elif val $==$ 'RS': ॥n return '110000' \n elif val == 'MWF': In

return '000110' $\backslash \mathrm{n} \quad$ elif $\mathrm{val}==$ return '010010' \n elif val $==$ return '001100' $\backslash \mathrm{n}$ elif $\mathrm{val}==$ return '100100'\n elif val $==$ return ' $101000 ' \backslash \mathrm{n}$ elif val $==$ return '010101' $\backslash \backslash \mathrm{n}$ elif val ==

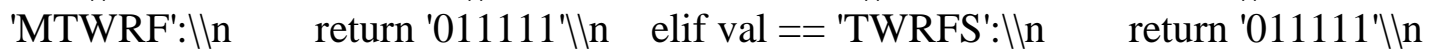
else:IIn return '010000'")

\# Process: Calculate Service Week

arcpy.CalculateField_management(Stores_With_SD, "ServiceWK", "Replace( [AW], ।" ।", ।"_।")", "VB", "')

\# Process: Add Volume arcpy.AddField_management(Stores_With_SW, "Volume", "DOUBLE", "", "", "4", "", "NULLABLE", "NON_REQUIRED", "")

\# Process: Calculate Volume arcpy.CalculateField_management(Stores_Volume, "Volume", "volume(

!ServiceWK! , !Size_1! , !Size_2! , !Size_3! )", "PYTHON_9.3", "def volume(

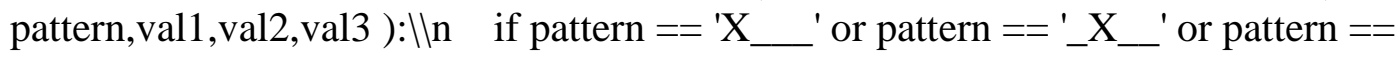

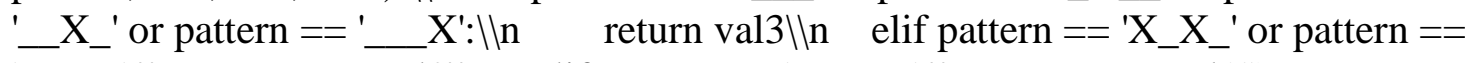
'_X_X': In return val2lln elif pattern == 'XXXX':॥n return val1") \# Process: Add DuplicateCheck arcpy.AddField_management(Stores_With_Vol, "DuplicateCheck", "SHORT", "", "", "4", "", "NULLABLE", "NON_REQUIRED", "")

\# Process: Calculate Duplicate arcpy.CalculateField_management(Stores_Check, "DuplicateCheck",

"find_duplicates( !Location_ID! )", "PYTHON_9.3", "d = \{\}॥n\\ndef

find_duplicates(val): In $\mathrm{d}[\mathrm{val}]=\mathrm{d}$.setdefault $(\mathrm{val},-1)+1 \backslash \mathrm{n} \quad$ return d[val]")

\# Process: Select Valid Stores

arcpy.Select_analysis(Stores_With_Check, Stores_Unique_ID, "DuplicateCheck = 0")

\# Process: Copy Unique Stores

arcpy.CopyFeatures_management(Stores_Unique_ID, Final_Stores, "", "0", "0", "0")

\# Create the orders for the present day. Parameters will take current week number and the day of the week into consideration.

if week_number == '1':

if Day == 'Monday':

arcpy.Select_analysis(Final_Stores, Day_Order, "ServiceDay LIKE ' $1^{\prime}$

AND ServiceWK LIKE 'X\%'"')

elif Day == 'Tuesday':

arcpy.Select_analysis(Final_Stores, Day_Order, "ServiceDay LIKE '___ 1_'

AND ServiceWK LIKE 'X\%"')

elif Day == 'Wednesday':

arcpy.Select_analysis(Final_Stores, Day_Order, "ServiceDay LIKE '____'

AND ServiceWK LIKE 'X\%"')

elif Day == 'Thursday': 
arcpy.Select_analysis(Final_Stores, Day_Order, "ServiceDay LIKE '____'

AND ServiceWK LIKE 'X\%'")

elif Day == 'Friday':

arcpy.Select_analysis(Final_Stores, Day_Order, "ServiceDay LIKE '_1__ '

AND ServiceWK LIKE 'X\%'")

elif Day == 'Saturday':

arcpy.Select_analysis(Final_Stores, Day_Order, "ServiceDay LIKE '1

AND ServiceWK LIKE 'X\%"')

elif week_number $==$ ' 2 ':

if Day $==$ 'Monday':

arcpy.Select_analysis(Final_Stores, Day_Order, "ServiceDay LIKE '

AND ServiceWK LIKE'_X\%'")

elif Day == 'Tuesday':

arcpy.Select_analysis(Final_Stores, Day_Order, "ServiceDay LIKE '___ 1_'

AND ServiceWK LIKE'_X\%'")

elif Day == 'Wednesday':

arcpy.Select_analysis(Final_Stores, Day_Order, "ServiceDay LIKE '__ 1 '

AND ServiceWK LIKE '_X\%'")

elif Day == 'Thursday':

arcpy.Select_analysis(Final_Stores, Day_Order, "ServiceDay LIKE '__ 1 _'

AND ServiceWK LIKE'_X\%"')

elif Day == 'Friday':

arcpy.Select_analysis(Final_Stores, Day_Order, "ServiceDay LIKE '_1__'

AND ServiceWK LIKE '_X\%'"')

elif Day == 'Saturday':

arcpy.Select_analysis(Final_Stores, Day_Order, "ServiceDay LIKE '1

AND ServiceWK LIKE'_X\%'")

elif week_number $==$ ' 3 ':

if Day == 'Monday':

arcpy.Select_analysis(Final_Stores, Day_Order, "ServiceDay LIKE

$1^{\prime}$

AND ServiceWK LIKE '_X\%'")

elif Day $==$ 'Tuesday':

arcpy.Select_analysis(Final_Stores, Day_Order, "ServiceDay LIKE '___1_'

AND ServiceWK LIKE '_X\%'")

elif Day $==$ 'Wednesday':

arcpy.Select_analysis(Final_Stores, Day_Order, "ServiceDay LIKE '__1_'

AND ServiceWK LIKE '_X\%'")

elif Day == 'Thursday':

arcpy.Select_analysis(Final_Stores, Day_Order, "ServiceDay LIKE '____'

AND ServiceWK LIKE '__X\%'")

elif Day == 'Friday':

arcpy.Select_analysis(Final_Stores, Day_Order, "ServiceDay LIKE '_1__'

AND ServiceWK LIKE '_X\%'")

elif Day == 'Saturday': 
arcpy.Select_analysis(Final_Stores, Day_Order, "ServiceDay LIKE '1

AND ServiceWK LIKE '__X\%'")

elif week_number $==$ ' 4 ':

if Day == 'Monday':

arcpy.Select_analysis(Final_Stores, Day_Order, "ServiceDay LIKE ' $1^{\prime}$

AND ServiceWK LIKE '__ X'")

elif Day == 'Tuesday':

arcpy.Select_analysis(Final_Stores, Day_Order, "ServiceDay LIKE '____'

AND ServiceWK LIKE '___ X'")

elif Day == 'Wednesday':

arcpy.Select_analysis(Final_Stores, Day_Order, "ServiceDay LIKE '___ _'

AND ServiceWK LIKE '__ X'")

elif Day == 'Thursday':

arcpy.Select_analysis(Final_Stores, Day_Order, "ServiceDay LIKE '__ _ '

AND ServiceWK LIKE '___ X'")

elif Day == 'Friday':

arcpy.Select_analysis(Final_Stores, Day_Order, "ServiceDay LIKE '_1__ '

AND ServiceWK LIKE '___ X'")

elif Day == 'Saturday':

arcpy.Select_analysis(Final_Stores, Day_Order, "ServiceDay LIKE '1___

AND ServiceWK LIKE '__ X'")

frequency $=$ arcpy.Frequency_analysis(Day_Order, freqTable, ["Route_"])

\# Capturing distinct route IDs from the present day orders.

cursor = arcpy.SearchCursor(frequency) \# Creating a frequency table that will allow

return all the routes with their occurrence number.

for row in cursor:

\# Reading the distinct route IDs using cursor

routeID $=($ row.getValue $($ freqField $))$

\# Creating orders based on the distinct route IDs

inOrders = "in_memoryllStores_" + str(routeID)

arcpy.Select_analysis(Day_Order, inOrders, "Route__ = " + str(routeID))

\# Check out the Network Analyst extension license

arcpy.CheckOutExtension("Network")

\# define workspace for the geodatabase that contains the network dataset and

select it

arcpy.env.workspace $=$ "C: $\backslash$ arcgis $\backslash$ Business Analystl|US_2014\\Datal|Streets

DatallNAVTEQ_2014_Q1_NA.gdb"

arcpy.env.overwriteOutput $=$ True

Routing_ND = "RoutinglRouting_ND"

\# Set local variables

outNALayerName = "Planned_Route_" + str(routeID)

impedanceAttribute $=$ "TravelTime"

distanceAttribute = "Miles"

Time_Field_Units = "Minutes" 
Distance_Field_Units $=$ "Miles"

\#Create a new Vehicle Routing Problem (VRP) layer.

outNALayer $=$ arcpy.na.MakeVehicleRoutingProblemLayer(Routing_ND,

outNALayerName, "TravelTime", "Miles", Time_Field_Units, Distance_Field_Units, "", "", "Medium", "Medium", "NO_UTURNS",

"'Avoid Carpool Roads';'Avoid Express

Lanes';'Avoid Gates';'Avoid Unpaved Roads';'Driving an Automobile';'Roads Under

Construction Prohibited';'Through Traffic Prohibited'",

"TRUE_LINES_WITH_MEASURES")

"USE_HIERARCHY", "',

\#Get the layer object from the result object. The VRP layer can now be

\#referenced using the layer object.

outNALayer $=$ outNALayer.getOutput $(0)$

\#Get the names of all the sublayers within the VRP layer.

subLayerNames $=$ arcpy.na.GetNAClassNames(outNALayer)

\#Stores the layer names that we will use later

ordersLayerName = subLayerNames["Orders"]

depotsLayerName $=$ subLayerNames["Depots"]

routesLayerName $=$ subLayerNames["Routes"]

\# Define workspace for the geodatabase that contains the depots or warehouse

details

arcpy.env.workspace $=$ "C:IIROARIIROARFleetManagement.sde"

arcpy.env.overwriteOutput $=$ True

inDepots = "OC_Depot"

\# Mapping or defining fields for the Depots layer

candidateDepotFields = arcpy.ListFields(inDepots)

depotFieldMappings = arcpy.na.NAClassFieldMappings(outNALayer,

depotsLayerName, False, candidateDepotFields)

depotFieldMappings["Name"].mappedFieldName = " WAREHOUSE"

depotFieldMappings["Description"].mappedFieldName = "Market"

depotFieldMappings["TimeWindowStart1"].defaultValue = "5 AM"

\# Loading the depot layer

arcpy.na.AddLocations(outNALayer, depotsLayerName, inDepots, depotFieldMappings, "100 Meters", "", "Routing_Streets

SHAPE;Routing_Streets_Override NONE;Routing_ND_Junctions NONE", "PRIORITY", "CLEAR", "NO_SNAP", "5 Meters", "EXCLUDE", "Routing_Streets

\#;Routing_Streets_Override \#;Routing_ND_Junctions \#")

\# Mapping or defining fields for the Orders layer

candidateOrderFields $=$ arcpy.ListFields(inOrders)

orderFieldMappings = arcpy.na.NAClassFieldMappings(outNALayer,

ordersLayerName, False, candidateOrderFields)

orderFieldMappings["Name"].mappedFieldName = "Location_I"

orderFieldMappings["Description"].mappedFieldName = "Location_N"

orderFieldMappings["ServiceTime"].mappedFieldName = "Fixed"

orderFieldMappings["ServiceTime"].defaultValue $=17$

orderFieldMappings["TimeWindowStart1"].mappedFieldName = "TW1_Open" 
orderFieldMappings["TimeWindowStart1"].defaultValue = '00:01:00 AM' orderFieldMappings["TimeWindowEnd1"].mappedFieldName = "TW1_Close" orderFieldMappings["TimeWindowEnd1"].defaultValue = '23:59:00 PM' orderFieldMappings["DeliveryQuantities"].mappedFieldName = "volume" \# Loading the order layer arcpy.na.AddLocations(outNALayer, ordersLayerName, inOrders, orderFieldMappings, "400 Meters", "", "Routing_Streets SHAPE;Routing_Streets_Override NONE;Routing_ND_Junctions NONE", "PRIORITY", "CLEAR", "NO_SNAP", "5 Meters", "EXCLUDE", "Routing_Streets \#;Routing_Streets_Override \#;Routing_ND_Junctions \#")

\# Calling the route details inRoutes = "OC_Truck"

\# Mapping or defining fields for the Orders layer candidateRouteFields = arcpy.ListFields(inRoutes) routeFieldMappings = arcpy.na.NAClassFieldMappings(outNALayer,

routesLayerName, False, candidateRouteFields)

routeFieldMappings["Name"].mappedFieldName = str(routeID) routeFieldMappings["StartDepotName"].mappedFieldName = "StartDepotName" routeFieldMappings["EndDepotName"].mappedFieldName = "EndDepotName" routeFieldMappings["Capacities"].defaultValue = 350

routeFieldMappings["EarliestStartTime"].defaultValue = '5:00:00 AM' routeFieldMappings["MaxOrderCount"].defaultValue $=25$

\# Loading the routes layer arcpy.na.AddLocations(outNALayer, routesLayerName, inRoutes, routeFieldMappings, "400 Meters", "", "Routing_Streets SHAPE;Routing_Streets_Override NONE;Routing_ND_Junctions NONE", "MATCH_TO_CLOSEST", "APPEND", "NO_SNAP", "5 Meters", "INCLUDE",

"Routing_Streets \#;Routing_Streets_Override \#;Routing_ND_Junctions \#") \# Solve the route layer arcpy.na.Solve(outNALayer, "HALT", "TERMINATE", "")

\# Convert the routes sublayers into feature classes outRouteFeatureClass = "in_memoryllPlanned_Route_" + str(routeID) outStopFeatureClass = "in_memoryl\Planned_Stops_" + str(routeID) \# Copy the solved routes and orders sublayers SolvedStops = arcpy.mapping.ListLayers(outNALayer, ordersLayerName) $[0]$ SolvedRoutes $=$ arcpy.mapping.ListLayers $($ outNALayer, routesLayerName $)[0]$ arcpy.management.CopyFeatures(SolvedStops, outStopFeatureClass) arcpy.management.CopyFeatures(SolvedRoutes, outRouteFeatureClass) \# Adds new field for date into the new fields in the planned route feature class arcpy.AddField_management(outRouteFeatureClass, "Date", "TEXT") date_time $=$ datetime.date.today () \# updates the route ID and today's date into the new fields in the planned route feature class cursor $=$ arcpy. UpdateCursor(outRouteFeatureClass) for row1 in cursor: row1.setValue("Name", routeID) 
row1.setValue("Date", str(date_time))

cursor.updateRow(row1)

\# Dissolves all the customer features into on single feature outStopFeatureClassDissolved = "in_memoryllPlanned_Final_Stops_" + str(routeID)

arcpy.Dissolve_management(outStopFeatureClass,

outStopFeatureClassDissolved)

\# Adds new field for date and route ID into the new fields in the planned store

feature class

arcpy.AddField_management(outStopFeatureClassDissolved, "Date", "TEXT") arcpy.AddField_management(outStopFeatureClassDissolved, "Name", "TEXT")

\# updates the route ID and today's date into the new fields in the planned route

feature class

cursor $=$ arcpy.UpdateCursor(outStopFeatureClassDissolved)

for row in cursor:

row.setValue("Date", str(date_time))

row.setValue("Name", routeID)

cursor.updateRow(row)

\# Appends the planned route and planned customers into the planned route and planned store feature classes respectively. arcpy.Append_management(outRouteFeatureClass, plannedRouteFC,

"NO_TEST","","")

arcpy.Append_management(outStopFeatureClassDissolved, plannedStoreFC, "NO_TEST","","")

\# Print confirmation of route creation

print " Planned route for the route ID " + str(routeID) + " is created"

\#Confirming the successful script run

print "Script completed successfully"

print(datetime.datetime.now()) 


\section{Appendix G. Script for Creating Actual Routes}

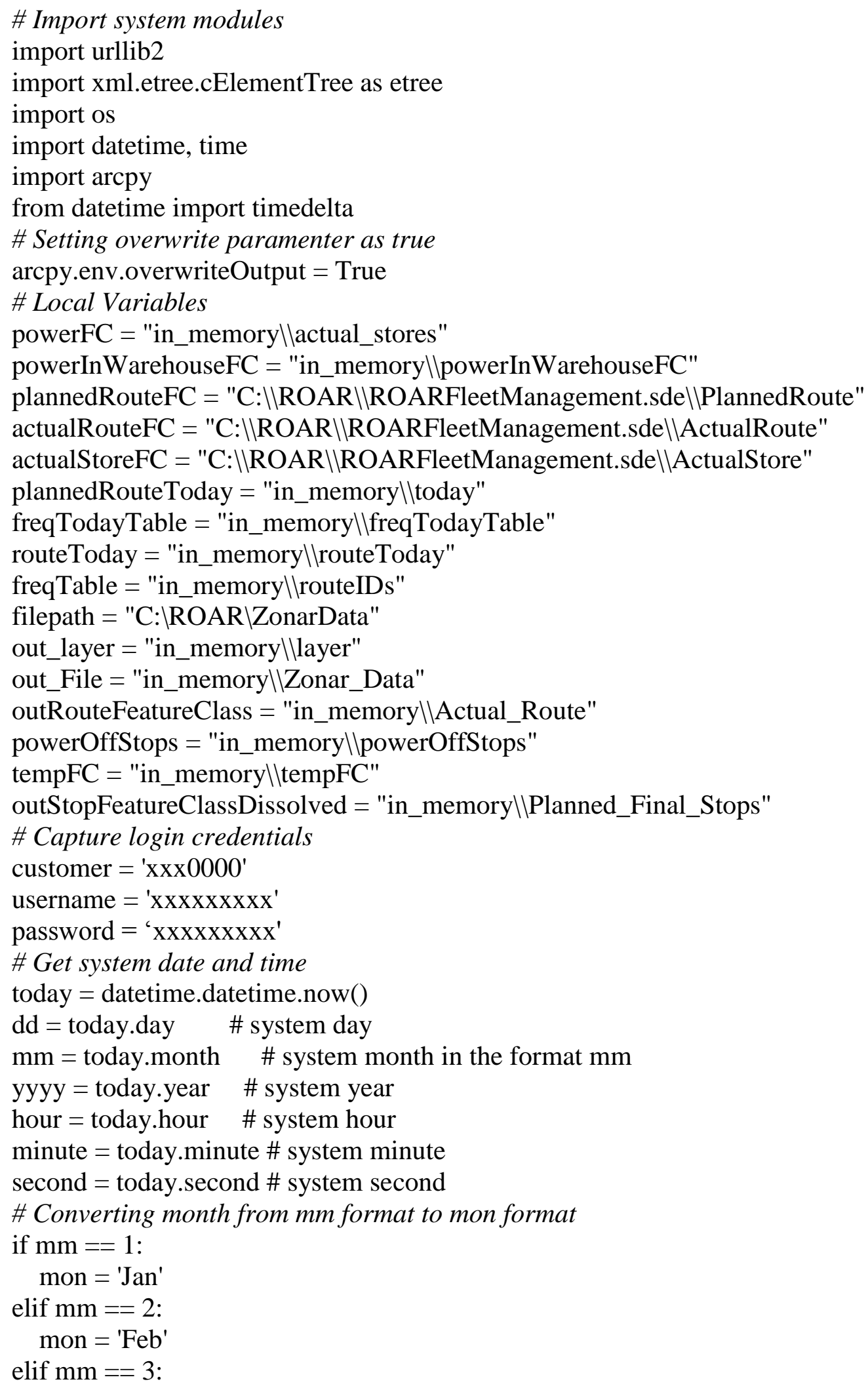




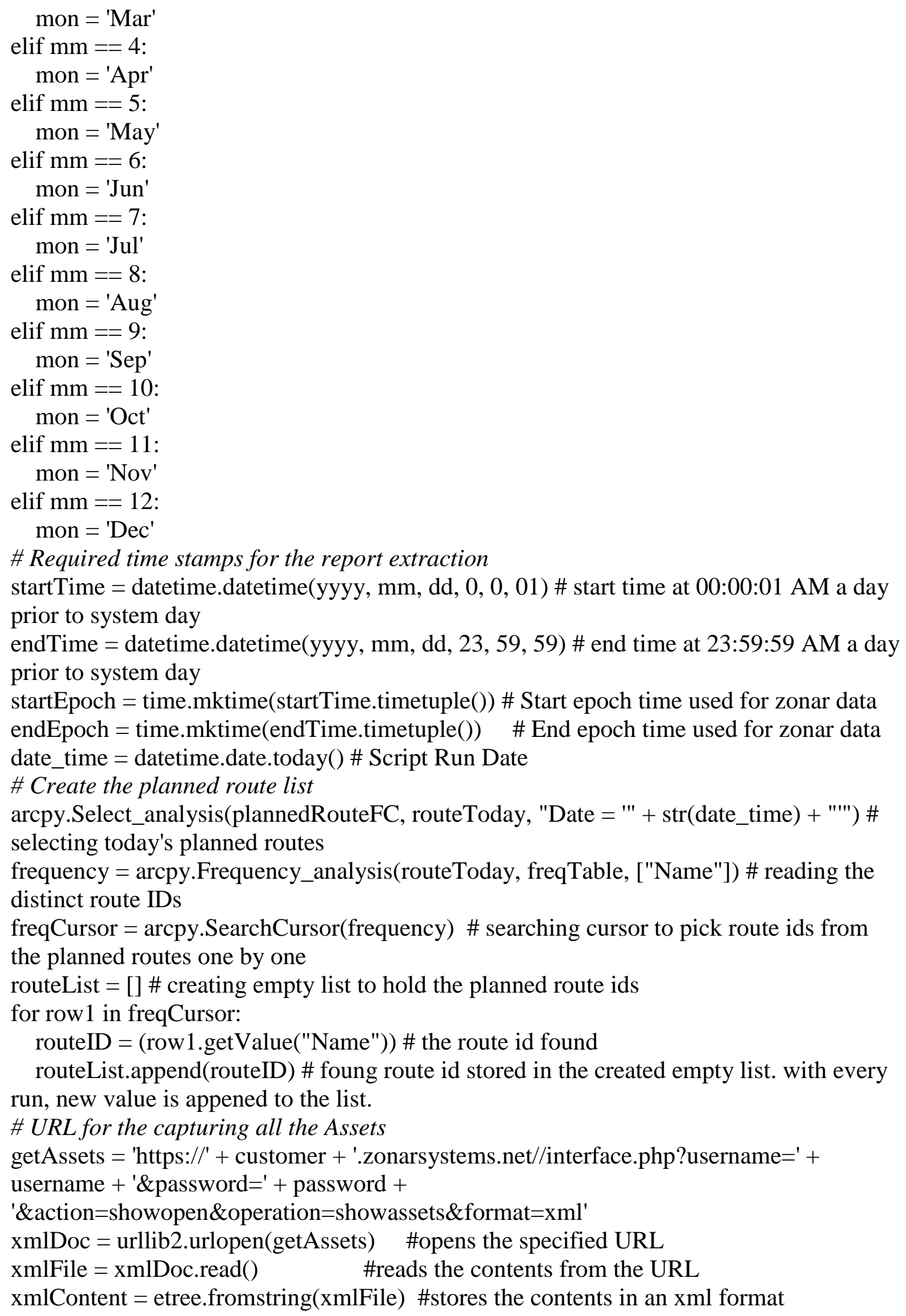


for assets in xmlContent.findall("asset"): the tag "asset".

location = assets.find("location")

inside each asset tag

if location.text == "Orange County": \# if the "location" tag value = 'Orange County', Script returs the fleet number that will be used in the report extraction AssetID = assets.attrib.get("id") \#Extracts the value of the "id" tag inside each asset tag

Fleet $=$ assets.find("fleet") asset tag

Fleet $=\operatorname{str}($ Fleet.text $)$

Fleet = Fleet.replace(" ", "_")

\#For loop runs through $\backslash$ all the blocks with

\#Extracts the value of the "location" tag

\#URL for API to extract actual route repots

downloadURL = 'https://' + customer +

'.zonarsystems.net//interface.php?username=' + username + '\&password=' + password +

'\&action=showposition\&operation=path\&reqtype=dbid\&target=' + $\operatorname{str}($ AssetID) +

'\&version=2\&starttime=' + str( startEpoch $)+$ '\&endtime=' + str(endEpoch $)+$

'\&logvers $=3.8 \&$ format $=\mathrm{csv}^{\prime}$

csvDoc $=$ urllib2.urlopen $($ downloadURL) \#run the link

csvReport $=$ csvDoc.read ()$\quad$ \#read the contents of the downloadable file

\#Generate the filename for storing the contents from the API

ZonarCSVFile $=$ customer + '-path-' + str(Fleet $)+$ '-' + str(dd) + '-' + mon + '-' +

str(yyyy) + '_' + str(hour) + '-' + str(minute) + '-' + str( second)

\#Generate the filepath with previously generated name and .csv extension

fileName = os.path.join(filepath, ZonarCSVFile +'.csv')

$\mathrm{f}=$ open(fileName, "a") \#create and open an empty file

f.write(csvReport) \#write the file with contents of the download link

f.close ()

\# Print confirmation of the .csv file creation

print " .csv file for the fleet " + Fleet + " is created"

\#Verify the contents of the .csv file.

\#If the report has no data other than the header then the process starts

\#from the beginning of the loops and picks the nest asset

$\mathrm{g}=$ open(fileName, "r")

num $=$ g.readlines ()

if len(num) $<=1$ :

continue

g.close ()

Fleet = Fleet.replace("(", "") \#replace the parenthesis beginning "(" in the fleet value with nothin ""

Fleet = Fleet.replace(")", "") \#replace the parenthesis beginning ")" in the fleet value with nothin ""

Route = Fleet.split("_")

Route $=$ Route[1]

the route id \#split the fleet number using '_.' ass a separator \#selects the second component for the fleet which is 
Route $=$ Route[:4] \#trims the route ID to first four characters

\#if the XY data exists in the .csv file then routes are created.

if Route in routeList:

arcpy.env.overwriteOutput $=$ True

\# input .csv file to be imported for feature class conversion

in_csv = fileName

\# Process: Make XY Event Layer

arcpy.MakeXYEventLayer_management(in_csv, "lon", "lat", out_layer,

"GEOGCS['GCS_WGS_1984',DATUM['D_WGS_1984',SPHEROID['WGS_1984',6378

137.0,298.257223563]],PRIMEM['Greenwich',0.0],UNIT['Degree',0.0174532925199433

]];-400 -400 1000000000;-100000 10000;-100000 10000;8.98315284119522E-

09;0.001;0.001;IsHighPrecision", "")

\# Process: Copy Features

arcpy.CopyFeatures_management(out_layer, out_File, "", "0", "0", "0")

\# Process: Connects the GPS points and make a single line

arcpy.PointsToLine_management(out_File, outRouteFeatureClass)

\# New field added: seconds

arcpy.AddField_management(out_File, "seconds", "LONG")

\# Calculated the new field "seconds"

timeSelect $=$ arcpy.SearchCursor(out_File) \# searches row by row features from

the out_file

for row6 in timeSelect: \# runs a loop for all the row in the search cursor

powerTime $=($ row6.getValue $($ "Time_PDT_") $)$ \# extracts the time in the original

format for the selected row

logTime = datetime.datetime.strptime (powerTime, '\%H:\%M:\%S').time() \#

converts the extracted time into $\mathrm{HH}: \mathrm{mm}$ :ss format

hour $=\log$ Time.hour \#extracted hour

minute $=\log$ Time.minute \# \#xtracted minute

second $=\log$ Time. second \#extracted second

totalTimeInSeconds $=($ hour*3600 $)+\left(\right.$ minute $\left.^{*} 60\right)+$ second $\#$ converts the time

into total seconds since the start of the day

timeUpdate $=$ arcpy.UpdateCursor(out_File, "Time_PDT_ $=$ "' + str(logTime)

+ "'") \# Updates the "seconds" field for the selected row

for row7 in timeUpdate:

row7.setValue("seconds", totalTimeInSeconds) \# sets the value

timeUpdate.updateRow(row7) \# saves the change

\# selects the logs only with the word "Power"

arcpy.Select_analysis(out_File, powerFC, "Log_Reason like '\%Power\%"')

\# selects the logs from the previous selction with the outside the RBDC OC zone arcpy.Select_analysis(powerFC, powerInWarehouseFC, "Zone <> 'RBDC OC'")

\# Creates an empty feature class with the schema of powerInWarehouseFC and

projection of actualRouteFC

arcpy.CreateFeatureclass_management("in_memory", "probableStops", "POINT", powerInWarehouseFC, "DISABLED", "DISABLED", actualRouteFC)

\# sets staring value for service time, engine power on time, and store count

actualServiceTime $=0$ 
actualStoreCount $=0$

onTime $=0$

\# Calculates the service time for a customer.

\#Considering the stop is made for a customer if the log reason was "Power off"

\# and the time gap between the power off and the very next power on was more 90 seconds. '\%Off\%'")

pOffCheck $=$ arcpy.SearchCursor(powerInWarehouseFC, "Log_Reason like

for row8 in pOffCheck:

offTime =row8.getValue("seconds")

pOnCheck $=$ arcpy.SearchCursor(powerInWarehouseFC, "Log_Reason like

'\%On\%' AND seconds > " + str(offTime))

pOnCount $=0$

for row9 in pOnCheck:

if $\mathrm{pOnCount}==0$ :

onTime = row9.getValue("seconds")

else:

break

pOnCount $+=1$

if onTime $>0$ :

serviceTime $=$ onTime-offTime

else:

serviceTime $=0$

if serviceTime > 90:

actualServiceTime $=$ actualServiceTime + serviceTime

actualStoreCount $=$ actualStoreCount +1

str(offTime))

arcpy.Select_analysis(powerInWarehouseFC, tempFC, "seconds =" +

"NO_TEST","","")

arcpy.Append_management(tempFC, "in_memoryllprobableStops",

\# Calculates the total distance travelled and the total time taken to finish the route.

\# distance value form the Last row give the total distance

\# time is calculated by subtracting the log time from last row from that of first

row.

\# calculates the time only if total row count in the csv is more than 3. Sometimes

a truck starts only to test the engine or some other reason but the GPS tracker creates a

$\log$.

\# all the time attributes have default of 0 seconds

$\#$ default distance $=0$ miles

getCount $=$ arcpy.GetCount_management(out_File)

rowCount $=\operatorname{int}($ getCount.getOutput $(0))$

if rowCount $<3$ :

actualDistance $=0$

actualServiceTime $=0$

actualTotalTime $=0$

actualTravelTime $=0$ 
actualStoreCount $=0$

continue

else:

rowList $=$ [row20 for row20 in arcpy.da.SearchCursor(out_File, "*")]

lastRow $=$ rowList $[-1]$

firstRow $=$ rowList $[0]$

actualDistance $=$ lastRow $[9]$

startFleetTime $=$ firstRow $[15]$

endFleetTime $=$ lastRow $[15]$

actualTotalTime $=$ endFleetTime - startFleetTime

actualTravelTime $=$ actualTotalTime - actualServiceTime

actualTotalTime $=$ float $($ actualTotalTime $) / 60$

actualTravelTime $=$ float $($ actualTravelTime $) / 60$

actualServiceTime $=$ float $($ actualServiceTime $) / 60$

\# new fields are added to store different calculated values.

arcpy.AddField_management(outRouteFeatureClass, "Name", "TEXT")

arcpy.AddField_management(outRouteFeatureClass, "Date", "TEXT")

arcpy.AddField_management(outRouteFeatureClass, "ActualStoresVisited", "SHORT") arcpy.AddField_management(outRouteFeatureClass, "ActualTravelTime",

"DOUBLE")

arcpy.AddField_management(outRouteFeatureClass, "ActualServiceTime",

"DOUBLE")

arcpy.AddField_management(outRouteFeatureClass, "ActualTotalTime",

"DOUBLE")

arcpy.AddField_management(outRouteFeatureClass, "ActualDistance",

"DOUBLE")

arcpy.AddField_management(outRouteFeatureClass, "PlannedTime",

"DOUBLE") arcpy.AddField_management(outRouteFeatureClass, "PlannedSerTime",

"DOUBLE")

arcpy.AddField_management(outRouteFeatureClass, "PlannedTravelTime", "DOUBLE") arcpy.AddField_management(outRouteFeatureClass, "PlannedDistance",

"DOUBLE")

arcpy.AddField_management(outRouteFeatureClass, "PlannedStoresCount", "SHORT")

arcpy.AddField_management(outRouteFeatureClass, "TotalTime", "DOUBLE") arcpy.AddField_management(outRouteFeatureClass, "TravelTime", "DOUBLE") arcpy.AddField_management(outRouteFeatureClass, "ServiceTime",

"DOUBLE")

arcpy.AddField_management(outRouteFeatureClass, "Distance", "DOUBLE")

arcpy.AddField_management(outRouteFeatureClass, "Stores", "SHORT")

arcpy.AddField_management(outRouteFeatureClass, "Efficiency", "FLOAT")

\# New added field are updated with the calculated distance, store count, name, today's date, all the attribute of time 
cursor $=$ arcpy.UpdateCursor(outRouteFeatureClass)

for row2 in cursor:

row2.setValue("Name", Route)

row2.setValue("Date", str(date_time))

row2.setValue("ActualStoresVisited", actualStoreCount)

row2.setValue("ActualTravelTime", actualTravelTime)

row2.setValue("ActualServiceTime", actualServiceTime)

row2.setValue("ActualDistance", actualDistance)

row2.setValue("ActualTotalTime", actualTotalTime)

cursor.updateRow(row2)

\# creates a table with details from the planned route generated for today

arcpy.Select_analysis(plannedRouteFC, plannedRouteToday, "Date = '" +

str(date_time $)+$ "'")

frequencyInsert $=$ arcpy.Frequency_analysis(plannedRouteToday,

freqTodayTable, ["Name", "TotalTime", "TotalDistance", "OrderCount",

"TotalOrderServiceTime", "TotalTravelTime" ])

\# extracts the planned route attributes

freqCursor $=$ arcpy.SearchCursor(frequencyInsert)

for row4 in freqCursor:

plannedRoute $=($ row4 .getValue $($ "Name") $)$

plannedTime $=($ row4.getValue $($ "TotalTime") $)$

plannedSerTime $=($ row4.getValue $(" T o t a l O r d e r S e r v i c e T i m e "))$

plannedTravelTime $=($ row4.getValue $($ "TotalTravelTime") $)$

plannedDistance $=($ row4.getValue $($ "TotalDistance" $))$

plannedStores $=($ row4.getValue $($ "OrderCount"))

\# updates the extracted values into the new fields

actualCursor $=$ arcpy.UpdateCursor(outRouteFeatureClass, "Name = "' +

str(plannedRoute $)+$ "' AND Date = '" + str(date_time $)+$ "'")

for row5 in actualCursor:

row5.setValue("PlannedStoresCount", plannedStores)

row5.setValue("PlannedTime", plannedTime)

row5.setValue("PlannedSerTime", plannedSerTime)

row5.setValue("PlannedTravelTime", plannedTravelTime)

row5.setValue("PlannedDistance", plannedDistance)

actualCursor.updateRow(row5)

\# calculates the KPIs

analysisSearchCursor $=$ arcpy.SearchCursor(outRouteFeatureClass) \# Creating a frequency table that will allow return all the routes with their occurrence number.

for row13 in analysisSearchCursor:

PT $=($ row 13.getValue("PlannedTime"))

PTT = (row13.getValue("PlannedTravelTime"))

PST = (row13.getValue("PlannedSerTime"))

AT = (row13.getValue("ActualTotalTime"))

ATT $=($ row13.getValue("ActualTravelTime"))

AST = (row 13.getValue("ActualServiceTime"))

$\mathrm{PD}=($ row13.getValue("PlannedDistance")) 


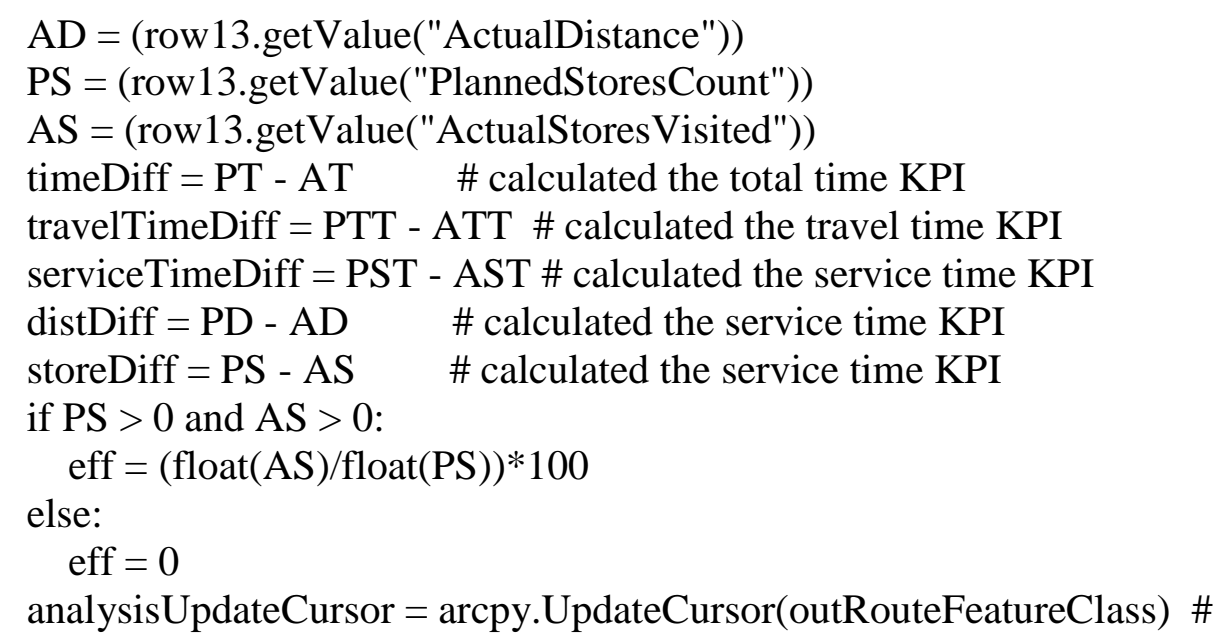

Creating a frequency table that will allow return all the routes with their occurrence number

for row 12 in analysisUpdateCursor:

row12.setValue("Efficiency", eff)

row12.setValue("TotalTime", timeDiff)

row12.setValue("TravelTime", travelTimeDiff)

row12.setValue("ServiceTime", serviceTimeDiff)

row12.setValue("Distance", distDiff)

row12.setValue("Stores", storeDiff)

analysisUpdateCursor.updateRow(row12)

\# Appends the generated actual route into the actual route feature class arcpy.Append_management(outRouteFeatureClass, actualRouteFC,

"NO_TEST","","")

\# Dissolves all the customer features into on single feature arcpy.Dissolve_management("in_memoryllprobableStops", outStopFeatureClassDissolved)

\# Adds new fields for today's date and route ID

arcpy.AddField_management(outStopFeatureClassDissolved, "Date", "TEXT") arcpy.AddField_management(outStopFeatureClassDissolved, "Name", "TEXT")

\# Calculates the new fields

cursor $=$ arcpy.UpdateCursor(outStopFeatureClassDissolved)

for row40 in cursor:

row40.setValue("Date", str(date_time))

row40.setValue("Name", Route)

cursor.updateRow(row40)

\# Appends the actual customers into the actual store feature class

arcpy.Append_management(outStopFeatureClassDissolved, actualStoreFC,

"NO_TEST","","')

\# Print confirmation of route creation

print " Actual route for the fleet " + Fleet + " is created"

\#Confirming the successful script run

print "Script completed successfully" 